\title{
CONSTANT TERM OF EISENSTEIN INTEGRALS ON A REDUCTIVE $p$-ADIC SYMMETRIC SPACE
}

\author{
JACQUES CARMONA AND PATRICK DELORME
}

\begin{abstract}
Let $H$ be the fixed point group of a rational involution $\sigma$ of a reductive $p$-adic group on a field of characteristic different from 2 . Let $P$ be a $\sigma$-parabolic subgroup of $G$, i.e. such that $\sigma(P)$ is opposite $P$. We denote the intersection $P \cap \sigma(P)$ by $M$.

Kato and Takano on one hand and Lagier on the other associated canonically to an $H$-form, i.e. an $H$-fixed linear form, $\xi$, on a smooth admissible $G$-module, $V$, a linear form on the Jacquet module $j_{P}(V)$ of $V$ along $P$ which is fixed by $M \cap H$. We call this operation the constant term of $H$-forms. This constant term is linked to the asymptotic behaviour of the generalized coefficients with respect to $\xi$.

P. Blanc and the second author defined a family of $H$-forms on certain parabolically induced representations, associated to an $M \cap H$-form, $\eta$, on the space of the inducing representation.

The purpose of this article is to describe the constant term of these $H$-forms.

Also it is shown that when $\eta$ is discrete, i.e. when the generalized coefficients of $\eta$ are square integrable modulo the center, the corresponding family of $H$-forms on the induced representations is a family of tempered, in a suitable sense, $H$-forms. A formula for the constant term of Eisenstein integrals is given.
\end{abstract}

\section{INTRODUCTION}

Let $\mathbf{F}$ be a nonarchimedean local field of characteristic different from 2 . The hypothesis on the characteristic of the residue field in the first version of the article has been removed (see Proposition 2.3 and Section [10).

Let $G$ be the set of $\mathbf{F}$-points of a connected reductive algebraic group defined over $\mathbf{F}$. Let $\sigma$ be a rational involution of $G$ defined over $\mathbf{F}$ and let $H$ be equal to the fixed point group of $G$ (a slightly weaker assumption is made in the main body of the article).

If $(\pi, V)$ is a smooth representation of $G$, an $H$-fixed element of the algebraic dual $V^{\prime}$ of $V$, i.e. $\xi \in V^{\prime H}$, will be called an $H$-form on $V$. We know (cf. 12, Theorem 4.5]) that if $V$ is of finite length the vector space of $H$-forms on $V$ is finite dimensional. We will denote the smooth dual of $V$ by $\check{V}$.

If $(\pi, V)$ is a smooth representation of $G$ and $P$ is a parabolic subgroup of $G$, we will denote the normalized Jacquet module along $P$ by $\left(\pi_{P}, V_{P}\right)$ and the canonical projection $V \rightarrow V_{P}$ by $j_{P}: V \rightarrow V_{P}$. For $v \in V$, we will also denote $j_{P}(v)$ by $v_{P}$.

Received by the editors December 8, 2011 and, in revised form, November 26, 2012.

2010 Mathematics Subject Classification. Primary $22 \mathrm{E} 50$.

Key words and phrases. Reductive group, nonarchimedean local field, symmetric space.

The second author has been supported by the program ANR-BLAN08-2-326851 and by the Institut Universitaire de France during the elaboration of this work. 
A parabolic subgroup of $G$ is called a $\sigma$-parabolic subgroup if and only if $P$ and $P^{-}:=\sigma(P)$ are opposite (studied by A. Helminck and Wang, A. Helminck and G. Helminck; cf. [18] and [17]). Then $M:=P \cap \sigma(P)$ is the $\sigma$-stable Levi subgroup of $P$ and $P H$ is open in $G$. Let $U$ be the unipotent radical of $P$.

Let $(\pi, V)$ be an admissible representation of $G$ and let $P$ be a $\sigma$-parabolic subgroup of $G$. Let $\pi^{\prime}$ be the dual representation of $\pi$ on $V^{\prime}$. Independently KatoTakano [20] and Lagier [23] have associated to each $H$-form $\xi$ on $V$ an $M \cap H$-form $j_{P^{-}} \xi$, also denoted by $\xi_{P^{-}}$, on $V_{P}$ such that, for all $v \in V$, there exists $\varepsilon$ satisfying:

$$
\delta_{P}^{-1 / 2}(a)\langle\xi, \pi(a) v\rangle=\left\langle\left(\xi_{P^{-}}, \pi_{P}(a) v_{P}\right\rangle, a \in A_{M}^{-}(\varepsilon),\right.
$$

where $A_{M}^{-}(\varepsilon)$ is some translate of the negative Weyl chamber in the maximal split torus, $A_{M}$, of the center of $M$ and $\delta_{P}$ is the modulus function of $P$. This is a characteristic property of $\xi_{P^{-}}$which describes the asymptotics of generalized coefficients. The linear form $\xi_{P^{-}}$will be called the constant term of $\xi$ along $P$.

By the Second Adjointness Theorem (Casselman and Bernstein; cf. [8], 2], [25. VI.9.6]) $(\check{V})_{P^{-}}$is canonically isomorphic to $\left(V_{P}\right)$, i.e. there exists a canonical nondegenerate pairing between $(\breve{V})_{P^{-}}$and $V_{P}$, denoted by $\langle., .\rangle_{P}$.

If $K$ is a compact open subgroup, let $e_{K}$ be the normalized Haar measure on $K$ and $e_{K} \xi$ be the smooth linear form defined by:

$$
\left\langle e_{K} \xi, v\right\rangle=\left\langle\xi, \pi\left(e_{K}\right) v\right\rangle, v \in V .
$$

We define the notion of a $(P, H)$-good compact open subgroup of $G$ (cf. (2.23)) . For $K$ a $(P, H)$-good compact open subgroup of $G$, one has:

$$
\left\langle\xi_{P^{-}}, v_{P}\right\rangle=\left\langle\left(e_{K} \xi\right)_{P^{-}}, v_{P}\right\rangle_{P}, v \in V^{K} .
$$

This property justifies the notation $\xi_{P^{-}}$. There exist arbitrary small $(P, H)$-good compact open subgroups of $G$ (Kato-Takano [20, Lemma 4.6] and Lagier [23, Theorem 1 (ii)]). Hence $\xi_{P^{-}}$is determined by the various $\left(e_{K} \xi\right)_{P^{-}}$.

P. Blanc and the second author defined families of $H$-forms on parabolically induced representations (cf. [3]). The purpose of this article is to describe the constant term of these $H$-forms.

For this we prepare several tools. First we introduce two operations on $H$-forms on induced representations.

The first one is denoted $\check{j}_{Q^{-}}$o (cf. Section 3.4). Let $P=M U$ be a parabolic subgroup of $G$, as above. Let $(\delta, E)$ be a smooth representation of finite length of $M$ and let $i_{P}^{G} E$ be the normalized parabolically induced representation. Our choice is such that the elements of $i_{P}^{G} E$ are left covariant $E$-valued functions and $G$ acts by the right regular representation. Let $\left(Q, Q^{-}\right)$be a pair of opposite parabolic subgroups of $M$. Let $P_{Q}=Q U \subset P, P_{Q^{-}}=Q^{-} U \subset P$. We define a homomorphism of $G$-modules,

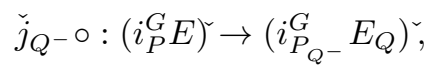

as the composition of four maps:

$$
\left(i_{P}^{G} E\right)^{\sim} \rightarrow i_{P}^{G} \check{E} \stackrel{f}{\rightarrow} i_{P_{Q^{-}}^{G}}^{G}\left((\check{E})_{Q^{-}}\right) \stackrel{g}{\rightarrow} i_{P_{Q^{-}}^{G}}^{G}\left(\left(E_{Q}\right)^{\curlyvee}\right) \rightarrow\left(i_{P_{Q^{-}}^{G}}^{G} E_{Q}\right)^{\sim},
$$

where the first and the last maps are deduced from a choice (cf. (3.10) ) of an isomorphism between the smooth dual of a parabolically induced representation and the parabolically induced representation of the smooth dual, where $f$ is given 
by the composition with $j_{Q^{-}}$and $g$ is the induced map of the isomorphism between $(\check{E})_{Q^{-}}$and $\left(E_{Q}\right)^{\sim}$ given by the Second Adjointness Theorem.

This operation on smooth linear forms easily extends to an $H$-form: one associates to an $H$-form, $\xi$, on $i_{P}^{G} E$, an $H$-form $\check{j}_{Q^{-}} \circ \xi$ on $i_{P_{Q^{-}}}^{G}\left(E_{Q}\right)$ (cf. Proposition 3.3 .

Let $P_{1}=M_{1} U_{1}$ be a parabolic subgroup of $G$ such that $P \subset P_{1}, M \subset M_{1}$ and such that $P_{1} H$ is open. The second operation is denoted $\check{r}_{M_{1}}$ (cf. Section 3.3). Let $\xi$ be an $H$-form on $i_{P}^{G} E$. Inducing in stages, $\xi$ is an $H$-form on $i_{P_{1}}^{G} E_{1}$ where $E_{1}:=i_{P \cap M_{1}}^{M_{1}} E$. The linear form $\xi$ might be viewed as an $E_{1}$-distribution which is right invariant under $H$ and left covariant under $P_{1}$. The restriction of this distribution to the open set $P_{1} H$ is simply a function. Its value at 1 is denoted $\check{r}_{M_{1}} \xi \in\left(E_{1}\right)^{\prime M_{1} \cap H}$.

The next tool is what we call the Generic Basic Geometric Lemma (cf. Proposition 4.8). Let $P=M U$ be a $\sigma$-parabolic subgroup of $G$. Let $(\delta, E)$ be a finite length smooth representation of $M$. Let $X(M)_{\sigma}$ be the identity component of the set of $\sigma$-antiinvariant elements of $X(M)$. If $\chi \in X(M)_{\sigma}$, we denote the space of the representation $\delta_{\chi}:=\delta \otimes \chi$ by $E_{\chi}$. If $x \in G$ and $X$ is a subset of $G, x X x^{-1}$ will be denoted by $x . X$. Then $x \delta$ is the representation of $x . M$ on $x E:=E$ such that, if $m \in M, x \delta\left(x m x^{-1}\right)=\delta(m)$. If there is no ambiguity, we will denote the bijective intertwining operator between $i_{P}^{G} E$ and $i_{x . P}^{G} x E$ by $\lambda(x) v(g)=v\left(x^{-1} g\right), g \in G$,. Also we will denote the transpose of the inverse of $\lambda(x)$ again by $\lambda(x)$.

Let $P^{\prime}=M^{\prime} U^{\prime}$ be another $\sigma$-parabolic subgroup of $G$. Then we have the following result that we call the Generic Basic Geometric Lemma (or rather its dual version; cf. Proposition 4.9).

Lemma. For a good choice $W\left(M^{\prime} \backslash G / M\right)$ of a set of representatives of $P^{\prime} \backslash G / P$, one defines successively for $w \in W\left(M^{\prime} \backslash G / M\right)$ :

$$
\begin{gathered}
X_{\chi, w}:=i_{M^{\prime} \cap w . P}^{M^{\prime}} w\left(\left(E_{\chi}\right)_{M \cap w^{-1} \cdot P^{\prime}}\right), \\
\tilde{P}_{w}=\left(M \cap w^{-1} \cdot P^{\prime-}\right) U, \tilde{P}_{w}^{\prime}=\left(M^{\prime} \cap w \cdot P\right) U^{\prime-} A A .
\end{gathered}
$$

Also, for $\chi$ in a suitable open dense subset of $X(M)_{\sigma}$ we define the transpose of the intertwining integral ${ }^{t} A\left(w \cdot \tilde{P}_{w}, \tilde{P}_{w}^{\prime}, w j_{M \cap w^{-1} \cdot P^{\prime}} \delta_{\chi}\right)$ and $\beta_{\chi, w}:\left(i_{P}^{G} E_{\chi}\right)^{\sim} \rightarrow\left(X_{\chi, w}\right)^{\sim}$ by:

$$
\beta_{\chi, w}=\check{r}_{M^{\prime}} \circ{ }^{t} A\left(w \cdot \tilde{P}_{w}, \tilde{P}_{w}^{\prime}, w j_{M \cap w^{-1} . P^{\prime}} \delta_{\chi}\right) \circ \lambda(w) \circ\left(\check{j}_{\left.M \cap w^{-1} . P^{\prime-} \circ\right)}\right.
$$

so that the linear map $\bigoplus_{w \in W\left(M^{\prime} \backslash G / M\right)} \beta_{\chi, w}:\left(i_{P}^{G} E_{\chi}\right)^{\sim} \rightarrow\left(\bigoplus_{w \in P^{\prime} \backslash G / P} X_{w}\right)^{\sim}$ goes through the quotient to an isomorphism:

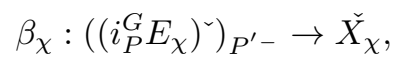

where $X_{\chi}=\bigoplus_{w \in W\left(M^{\prime} \backslash G / M\right)} X_{\chi, w}$.

The proof of the Generic Basic Geometric Lemma requires the study of bijectivity of intertwining integrals and the notion of an infinitesimal character linked to the Bernstein center (cf. Section 9.2). This notion allows us to show that, for $\chi$ in an open dense subset of $X(M)_{\sigma}$, two distinct $X_{\chi, w}$ do not have irreducible subquotients in common. The end of the proof uses the Basic Geometric Lemma which describes the graded object associated to a natural filtration of the Jacquet module of a parabolically induced representation.

From our isomorphism $\beta_{\chi}:\left(\left(i_{P}^{G} E_{\chi}\right)^{`}\right)_{P^{\prime-}} \rightarrow \check{X}_{\chi}, X_{\chi}$ is identified with $\left(i_{P}^{G} E_{\chi}\right)_{P^{\prime}}$, by the Second Adjointness Theorem. Hence, if $\xi$ is an $H$-form on $i_{P}^{G} E_{\chi}, \xi_{P^{\prime}-}$ 
is identified with a linear form denoted again by $\xi_{P^{\prime}-}$ on $X_{\chi}$, with components $\xi_{P^{\prime-},{ }_{w}} \in\left(X_{\chi, w}\right)^{\prime}$.

By reduction to the $e_{K} \xi$, and by unwinding the definitions, one proves a preliminary result (cf. Theorem 4.11):

Theorem 1. For $\chi$ in an open dense subset of $X(M)_{\sigma}$,

$$
\xi_{P^{\prime-}, w}=\check{r}_{M^{\prime}} \circ{ }^{t} A\left(w \cdot \tilde{P}_{w}, \tilde{P}_{w}^{\prime}, w j_{M \cap w^{-1} \cdot P^{\prime}} \delta\right) \circ \lambda(w) \circ \check{j}_{M \cap w^{-1} \cdot P^{\prime-}} \circ \xi .
$$

To simplify the exposition, we assume, only in the introduction, that there is only one open $(P, H)$-double coset, $P H$. A theorem due to Blanc and the second author (cf. [3, Theorem 2.8]) can be stated as follows. For $\eta \in E^{\prime} M \cap H$, there exists a unique rational map on $X(M)_{\sigma}, \chi \rightarrow \xi\left(P, \delta_{\chi}, \eta\right) \in\left(i_{P}^{G} \delta_{\chi}\right)^{\prime} H$, with $\check{r}_{M} \xi\left(P, \delta_{\chi}, \eta\right)=\eta$. The proof of this theorem adapts to the weaker hypothesis on $\mathbf{F}$ in this article.

Let $Q$ be a $\sigma$-parabolic subgroup of $G$ whose $\sigma$-stable Levi subgroup is equal to $M$. Then one sees (cf. Proposition 6.2) that there is a rational map on $X(M)_{\sigma}$ (the analogue of the $B$-matrices of van den Ban, [1] $), \chi \mapsto B\left(Q, P, \delta_{\chi}\right) \in \operatorname{End}\left(E^{\prime} M \cap H\right)$, such that:

$$
{ }^{t} A\left(P, Q, \delta_{\chi}\right) \xi\left(P, \delta_{\chi}, \eta\right)=\xi\left(P, \delta_{\chi}, B\left(Q, P, \delta_{\chi}\right) \eta\right)
$$

Let $(\pi, V)$ be a smooth representation of $G$ and let $\xi$ be an $H$-form on $V$. For $v \in V$, we denote the smooth function on $H \backslash G$ defined by:

$$
c_{\xi, v}(H g)=\langle\xi, \pi(g) v\rangle, g \in G,
$$

by $c_{\xi, v}$. If $\pi$ is of finite length, hence admissible, $\xi$ is said to be cuspidal or $H$ cuspidal if one of the equivalent statements holds (Kato-Takano [20]):

1) $\xi_{P^{-}}=0$ for every proper $\sigma$-parabolic subgroup, $P$, of $G$.

2) For all $v \in V$, the function $g \mapsto\langle\xi, \pi(g) v\rangle$ has a compact support modulo $H Z$, where $Z$ is the center of $G$.

Assuming that $(\pi, V)$ is irreducible and unitary, an $H$-form is said to be discrete if the $c_{\xi, v}$ are square integrable on $H \backslash G / A_{G}$. Kato and Takano (cf. [21, Theorem 4.7]) have characterized a discrete $H$-form $\xi$ by a criterion analogous to a criteria of Casselman [8, Theorem 4.4.6] for square integrable representations of the group. It involves a condition on $j_{P^{-}} \xi$ for each $\sigma$-parabolic subgroup of $G, P$. We define the tempered $H$-forms by a similar property (cf. Definition 7.6 ). One has the following result (cf. Theorems [7.4, 7.5, 7.8).

Theorem 2. Let $\eta \in E^{\prime M \cap H}$. If $\chi \in X(M)_{\sigma}$ we denote the $H$-form $\xi\left(P, \delta_{\chi}, \eta\right)$ by $\xi_{\chi}$, when it is defined.

(i) Let us assume that $\left(\xi_{\chi}\right)_{P^{\prime-}, w}$ is nonidentically zero when $\chi$ varies in $X(M)_{\sigma}$. Then one may change $w$ in the same double $\left(P^{\prime}, P\right)$-coset such that:

a) $M^{\prime} \cap w . P$ is a $\sigma$-parabolic subgroup of $M^{\prime}, M \cap w^{-1} \cdot P^{\prime}$ is a $\sigma$-parabolic subgroup of $M$, and $\tilde{P}_{w}^{\prime}, P_{w}$ and $w . \tilde{P}_{w}$ are $\sigma$-parabolic subgroups of $G$.

b) The exponents (cf. Definition 7.3 ) of $\xi_{\chi}$ along $P^{\prime}$ are explicitly controlled by the exponents of $\eta$ along certain $\sigma$-parabolic subgroups of $M$.

(ii) With these choices of $w$, if $\eta$ is cuspidal, one has $w \cdot M \subset M^{\prime}, M \cap w^{-1} \cdot P^{\prime-}=$ $M$, so that $\tilde{P}_{w}=P$ and

$$
\left(\xi_{\chi}\right)_{P^{\prime-}, w}=\xi\left(M^{\prime} \cap w \cdot P, w \delta_{\chi}, B\left(\tilde{P}_{w}^{\prime}, w \cdot P, w \delta_{\chi}\right) \eta\right) .
$$

(iii) If $\eta$ is discrete and $\chi$ is unitary, then $\xi_{\chi}$ is tempered. The notion of a weak constant term of tempered $H$-forms is introduced and computed for $\xi_{\chi}$.

(iv) The B-matrices preserve cuspidal (resp., discrete) $M \cap H$-forms. 
Remark. (a) As expected, when $\eta$ is cuspidal and $P^{\prime}$ is too small, $\xi_{P^{\prime}-}$ vanishes, by (ii).

(b) The analogue of part (iii) for real groups is the main result of [10] which plays an important role in the proof of the Plancherel formula (cf. [11]). The long proof used the description of the relative discrete series by Oshima and Matsuki. Our proof here does not need such knowledge. It would be interesting if one could find a proof of this result of [10], avoiding the description of the relative discrete series.

Let us describe the two main key lemmas of the article and let us explain how they lead to (i) a) of the theorem above.

First key lemma (cf. Lemma 5.1). Let $P$ be a parabolic, not necessarily a $\sigma$ parabolic, subgroup of $G$. Let $A_{0}$ be a $\sigma$-stable maximal split torus of $P$, which exists, and let $M$ be a Levi subgroup of $P$ with $A_{0} \subset M$. Let $\delta$ be a smooth representation of $M$ of finite length.

Let $\left(\xi_{\chi}\right)$ be a smooth family of $H$-forms on $i_{P}^{G}\left(\delta_{\chi}\right)$, with $\chi$ in a neighborhood of 1 in a complex subtorus $X$ of $X(M)$.

Let us assume that PH is contained and open in the support of the family $\left(\xi_{\chi}\right)$.

Then the elements of $X$ are antiinvariant under $\sigma$. Moreover, if there exists $\chi$ strictly $P$-dominant in $X$, then $P$ is a $\sigma$-parabolic subgroup of $G$.

Applying this to the family $\left(\left(\xi_{\chi}\right)_{P^{\prime}-, w}\right.$ ) (with $G$ replaced by $M^{\prime}$, and $P$ by a suitable conjugate in $M^{\prime}$ of $M^{\prime} \cap w \cdot P$ ), one sees why, in Theorem 2 (i) a), one can take $M^{\prime} \cap w . M$ to be a $\sigma$-parabolic subgroup of $M^{\prime}$. Then it is possible to refine the choice of $w$ to get (i) a).

Let us explain how one gets assertion (a) of the above remark and let us assume now that $\eta$ is cuspidal. If $M \cap w^{-1} \cdot P^{\prime}$ is different from $M$, it is not possible to see directly that $\left(\xi_{\chi}\right)_{P^{\prime-}, w}$ is zero. What is easily seen in that case, from the cuspidality of $\eta$, is that $\check{j}_{M \cap w^{-1} . P^{\prime}} \circ \xi_{\chi}$ vanishes on the open $\left(\tilde{P}_{w}, H\right)_{\chi}$-double cosets, hence its support has an empty interior.

The second key lemma (cf. Lemma 5.4) describes the effect of intertwining integrals on the support of certain $H$-forms. It was suggested by a lemma of Matsuki (cf. [24, Lemma 3]) on the orbit closures of orbits of parabolic subgroups on a real reductive symmetric space. Then one sees, using the second key lemma (see the proof of Theorem 7.4 (iii)), that for $\chi$ in an open dense subset of $X(M)_{\sigma}$, the element

$$
{ }^{t} A\left(w \cdot \tilde{P}_{w}, \tilde{P}_{w}^{\prime}, w j_{M \cap w^{-1} . P^{\prime}} \delta\right) \circ \lambda(w) \circ \check{j}_{M \cap w^{-1} . P^{\prime}-} \circ \xi_{\chi}
$$

of $\left(i_{\tilde{P}_{w}^{\prime}}^{G}\left(w j_{M \cap w^{-1} . P^{\prime}} \delta_{\chi}\right)\right)^{\prime}$ has support with an empty interior.

Applying $\check{r}_{M^{\prime}}$ to something which vanishes on all open $\left(\tilde{P_{w}^{\prime}}, H\right)$-double cosets, you get something which vanishes on all open $\left(M^{\prime} \cap w \cdot P, M^{\prime} \cap H\right)$-double cosets. The last ingredient to prove assertion (a) of the above Remark is that for $\chi$-generic, an $H$-form on $i_{P}^{G} E_{\chi}$ is determined by its restriction to open $(P, H)$-double cosets (cf. [3]). This is applied to $M^{\prime}$ instead of $G$.

The computation of $\left(\xi_{\chi}\right)_{P^{\prime}-, w}$ when $M \cap w^{-1} \cdot P^{\prime}=M$ follows from Theorem 1 . The last statement, which says that $B$-matrices preserve cuspidal $M \cap H$-forms, comes from the hereditary properties of $j_{P^{\prime}}$ and the fact observed in the Remark, that for $P^{\prime}$ small enough, $j_{P^{\prime}-} \xi=0$.

Theorem 2, together with the determination of part of the Casselman pairing for parabolically induced representations (cf. Lemma 8.3), leads to the determination 
of the constant term and the weak constant term of Eisenstein integrals for $p$-adic reductive symmetric spaces in terms of the corresponding $C$-functions (cf. Theorem 8.4).

The role of this type of result might be seen in the $p$-adic case in 29] for the work of Harish-Chandra on the Plancherel formula for $p$-adic groups, and in [13, 14 for Whittaker functions.

\section{NotATION}

2.1. Reductive $p$-adic groups. If $E$ is a vector space, $E^{\prime}$ will denote its dual. If $E$ is real, $E_{\mathbb{C}}$ will denote its complexification.

If $G$ is a group, $g \in G$ and $X$ is a subset of $G, g . X$ will denote $g X g^{-1}$. If $J$ is a subgroup of $G, g \in G$ and $(\pi, V)$ is a representation of $J, V^{J}$ will denote the space of invariant elements of $V$ under $J$ and $(g \pi, g V)$ will denote the representation of $g . J$ on $g V:=V$ defined by:

$$
(g \pi)\left(g x g^{-1}\right):=\pi(x), x \in J .
$$

We will denote the dual representation of a representation $(\pi, V)$ of $G$ in the algebraic dual vector space $V^{\prime}$ of $V$ by $\left(\pi^{\prime}, V^{\prime}\right)$. If $V$ is a vector space of vector-valued functions on $G$ which is invariant under right (resp., left) translations, we will denote the right regular representation of $G$ in $V$ by $\rho$ (resp., $\lambda$ ).

If $G$ is locally compact, $d_{l} g$ will denote a left invariant Haar measure on $G$ and $\delta_{G}$ will denote the modulus function.

Let $\mathbf{F}$, be a nonarchimedean local field. Unless specified we assume:

The characteristic of $\mathbf{F}$ is different from 2 .

Let $|\cdot|_{\mathbf{F}}$ be the absolute value of $\mathbf{F}$.

One considers various algebraic groups defined over $\mathbf{F}$, and a sentence like:

"let $A$ be a split torus" will mean "let $A$ be the group of $\mathbf{F}$-points of a torus, $\underline{A}$, defined and split over $\mathbf{F} "$.

With these conventions, let $G$ be a connected reductive linear algebraic group.

Let $A$ be a split torus of $G$. Let $X_{*}(A)$ be the group of one-parameter subgroups of $A$. This is a free abelian group of finite type. Such a group will be called a lattice. One fixes a uniformizing element $\varpi$ of $\mathbf{F}$ :

One denotes by $\Lambda(A)$ the image of $X_{*}(A)$ in $A$ under the morphism of groups $\underline{\lambda} \mapsto \underline{\lambda}(\varpi)$.

Under this morphism $\Lambda(A)$ is isomorphic to $X_{*}(A)$.

If $\left(P, P^{-}\right)$are two opposite parabolic subgroups of $G$, we will denote their common Levi subgroup by $M$ and the maximal split torus of its center by $A_{M}$ or $A$. We denote the unipotent radical of $P$ (resp., $P^{-}$) by $U$ (resp., $U^{-}$).

Let $A^{-}$(or $A_{P}^{-}$) be the set of $P$-antidominant elements in $A$. More precisely, if $\Sigma(P, A)$ is the set of roots of $A$ in the Lie algebra of $P$, and $\Delta(P, A)$ is the set of simple roots, one has:

$$
A^{-}=\left\{a \in A \|\left.\alpha(a)\right|_{\mathbf{F}} \leq 1, \quad \alpha \in \Delta(P, A)\right\} .
$$

Also one defines for $\varepsilon>0$ :

$$
A^{-}(\varepsilon)=\left\{\left.a \in A|| \alpha(a)\right|_{\mathbf{F}} \leq \varepsilon, \alpha \in \Delta(P, A)\right\} .
$$


If $J$ is an algebraic group, one denotes by $\operatorname{Rat}(J)$ the group of its rational characters defined over $F$. Let us define:

$$
\mathfrak{a}_{G}=\operatorname{Hom}_{\mathbb{Z}}(\operatorname{Rat}(G), \mathbb{R}) .
$$

The restriction of rational characters from $G$ to $A_{G}$ induces an isomorphism:

$$
\operatorname{Rat}(G) \otimes_{\mathbb{Z}} \mathbb{R} \simeq \operatorname{Rat}\left(A_{G}\right) \otimes_{\mathbb{Z}} \mathbb{R} .
$$

Notice that $\operatorname{Rat}\left(A_{G}\right)$ appears as a generating lattice in the dual space $\mathfrak{a}_{G}^{\prime}$ of $\mathfrak{a}_{G}$ and

$$
\mathfrak{a}_{G}^{\prime} \simeq \operatorname{Rat}(G) \otimes_{\mathbb{Z}} \mathbb{R} .
$$

One has the canonical map $H_{G}: G \rightarrow \mathfrak{a}_{G}$ which is defined by:

$$
e^{\left\langle H_{G}(x), \chi\right\rangle}=|\chi(x)|_{\mathbf{F}}, x \in G, \chi \in \operatorname{Rat}(G) .
$$

The kernel of $H_{G}$, which is denoted by $G^{1}$, is the intersection of the kernels of the characters of $G,|\chi|_{\mathbf{F}}, \chi \in \operatorname{Rat}(G)$. One defines

$$
X(G)=\operatorname{Hom}\left(G / G^{1}, \mathbb{C}^{*}\right),
$$

which is the group of unramified characters of $G$. One will use similar notation for Levi subgroups of $G$. One denotes by $\mathfrak{a}_{G, \mathbf{F}}$, resp., $\tilde{\mathfrak{a}}_{G, \mathbf{F}}$, the image of $G$, resp., $A_{G}$, under $H_{G}$. Then $G / G^{1}$ is isomorphic to the lattice $\mathfrak{a}_{G, \mathbf{F}}$.

There is a surjective map:

$$
\left(\mathfrak{a}_{G}^{\prime}\right)_{\mathbb{C}} \rightarrow X(G) \rightarrow 1
$$

denoted by $\nu \mapsto \chi_{\nu}$ which associates to $\chi \otimes s$, with $\chi \in \operatorname{Rat}(G), s \in \mathbb{C}$, the character $g \mapsto|\chi(g)|_{\mathbf{F}}^{s}$ (cf. [29, I.1.(1)]). In other words,

$$
\chi_{\nu}(g)=e^{\left\langle\nu, H_{G}(g)\right\rangle}, g \in G, \nu \in\left(\mathfrak{a}_{G}^{\prime}\right)_{\mathbb{C}} .
$$

The kernel is a lattice and it defines a structure of a complex algebraic variety on $X(G)$ of dimension $\operatorname{dim}_{\mathbb{R}} \mathfrak{a}_{G}$. Moreover $X(G)$ is an abelian complex Lie group whose Lie algebra is equal to $\left(\mathfrak{a}_{G}^{\prime}\right)_{\mathbb{C}}$.

If $\chi$ is an element of $X(G)$, let $\nu$ be an element of $\mathfrak{a}_{G, \mathbb{C}}^{\prime}$ such that $\chi_{\nu}=\chi$. The real part $\operatorname{Re}(\nu) \in \mathfrak{a}_{G}^{\prime}$ is independent from the choice of $\nu$. We will denote it by $\operatorname{Re}(\chi)$. If $\chi \in \operatorname{Hom}\left(G, \mathbb{C}^{*}\right)$ is continuous, the character $|\chi|$ of $G$ belongs to $X(G)$. One sets $\operatorname{Re}(\chi)=\operatorname{Re}(|\chi|)$. Similarly, if $\chi \in \operatorname{Hom}\left(A_{G}, \mathbb{C}^{*}\right)$ is continuous, the character $|\chi|$ of $A_{G}$ extends uniquely to an element of $X(G)$ with values in $\mathbb{R}^{*+}$, that we will denote again by $|\chi|$, and one sets $\operatorname{Re}(\chi)=\operatorname{Re}(|\chi|)$.

If $P$ is a parabolic subgroup of $G$ with Levi subgroup $M$ we set:

$$
\mathfrak{a}_{P}=\mathfrak{a}_{M}, H_{P}=H_{M} .
$$

The inclusions $A_{G} \subset A_{M} \subset M \subset G$ determine a surjective morphism $\mathfrak{a}_{M, \mathbf{F}} \rightarrow \mathfrak{a}_{G, \mathbf{F}}$, resp., an injective morphism $\tilde{\mathfrak{a}}_{G, \mathbf{F}} \rightarrow \tilde{\mathfrak{a}}_{M, \mathbf{F}}$, which extends uniquely to a surjective linear map between $\mathfrak{a}_{M}$ and $\mathfrak{a}_{G}$, resp., an injective map between $\mathfrak{a}_{G}$ and $\mathfrak{a}_{M}$. The second map allows us to identify $\mathfrak{a}_{G}$ with a subspace of $\mathfrak{a}_{M}$, and the kernel of the first one, $\mathfrak{a}_{M}^{G}$, satisfies:

$$
\mathfrak{a}_{M}=\mathfrak{a}_{M}^{G} \oplus \mathfrak{a}_{G} .
$$

Let us denote the set of restrictions to $M$ of elements of $X(G)$ by $X(G \mid M)$. Then $X(G \mid M)$ is the analytic subgroup of $X(M)$ with Lie algebra $\left(\mathfrak{a}_{G}^{\prime}\right)_{\mathbb{C}} \subset\left(\mathfrak{a}_{M}^{\prime}\right)_{\mathbb{C}}$. This follows easily from (2.9) and (2.10). Moreover:

$$
X(G \mid M) \text { is a closed subgroup of } X(M) .
$$


This will be seen by writing $X(G)=X(G)_{u} X(G)^{+}$, where $X(G)_{u}$ is the compact group of unitary characters in $X(G)$ and $X(G)^{+}$is the group of elements in $X(G)$ which are strictly positive. The group $X(G)_{u}$ is compact and has compact image. The group $X(G)^{+}$is isomorphic to a vector subgroup, and the restriction, which is a morphism of Lie groups, determines an isomorphism of $X(G)^{+}$to a connected Lie subgroup of $X(M)^{+}$: the restriction of an element of $X(G)^{+}$trivial on $A_{G}$ is trivial by what has been said above. Hence the image of $X(G)^{+}$is closed. This implies our claim on $X(G \mid M)$.

One has (cf. [12, (4.5))

The map $\Lambda\left(A_{G}\right) \rightarrow G / G^{1}$ is injective and allows us to identify $\Lambda\left(A_{G}\right)$ with a subset of $\mathfrak{a}_{G}$.

Let $A$ be a maximal split torus of $G$ and let $M_{0}$ be the centralizer of $A$ in $G$. We fix a $W$-invariant scalar product on $\mathfrak{a}:=\mathfrak{a}_{M_{0}}$, where $W$ is the Weyl group of $(G, A)$. Then $\mathfrak{a}_{G}$ is identified with the fixed point set of $\mathfrak{a}$ under $W$ and $\mathfrak{a}^{G}$ is an invariant subspace of $\mathfrak{a}$ under $W$ which is supplementary to $\mathfrak{a}_{G}$. Hence it is the orthogonal subspace to $\mathfrak{a}_{G}$ in $\mathfrak{a}$. The space $\mathfrak{a}_{G}^{\prime}$ might be viewed as a subspace of $\mathfrak{a}^{\prime}$ by (2.10). More generally let $M$ be a Levi subgroup of $G$ which contains $A$. From (2.10) applied to $M$ instead of $G$ and to $M_{0}$ instead of $M$, one gets a decomposition $\mathfrak{a}=\mathfrak{a}_{M_{0}}^{M} \oplus \mathfrak{a}_{M}$. From the $W$-invariance of the scalar product one gets:

The decomposition $\mathfrak{a}=\mathfrak{a}_{M_{0}}^{M} \oplus \mathfrak{a}_{M}$ is an orthogonal decomposition. The

space $\mathfrak{a}_{M}^{\prime}$ appears as a subspace of $\mathfrak{a}^{\prime}$ and, in the identification of $\mathfrak{a}$ with

$\mathfrak{a}^{\prime}$ given by the scalar product, $\mathfrak{a}_{M}^{\prime}$ is identified with $\mathfrak{a}_{M}$.

If $\nu \in \mathfrak{a}^{\prime}$, the parabolic subgroup of $G$ whose Lie algebra is equal to the sum of the $A$-weight spaces for the weights $\alpha$ which are either equal to zero or to a root $\alpha$ such that $(\nu, \alpha) \geq 0$, is denoted by $P_{\nu}$. Then one has:

The parabolic subgroups of $G, P_{\nu}$ and $P_{-\nu}$ are opposite.

If $\rho_{P} \in \mathfrak{a}^{\prime}$ is the half sum of the $A$-roots of $A$ in the Lie algebra of $P$, then the following is clear:

$$
P=P_{\rho_{P}} .
$$

Let $\underline{G}$ be the algebraic group defined over $\mathbf{F}$ whose group of $\mathbf{F}$-points is $G$. Let $\sigma$ be a rational involution of $\underline{G}$ defined over $\mathbf{F}$. Let $H$ be the group of $\mathbf{F}$-points of an open $\mathbf{F}$-subgroup of the fixed point set of $\sigma$. We will also denote the restriction of $\sigma$ to $G$ by $\sigma$.

A split torus of $G, A$, is said to be $\sigma$-split if $A$ is contained in the set of elements of $G$ which are antiinvariant under $\sigma$.

Notice that our terminology differs from other authors ([18, 17, 20]). Let $A$ be a $\sigma$-invariant maximal split torus of $G$. We fix a scalar product on $\mathfrak{a}$ which is invariant under $\sigma$ and the action of the Weyl group of $G$ with respect to $A$. This is possible because $\sigma$ normalizes $A$, hence its normalizer and the Weyl group of $G$ with respect to $A$.

Let $A^{\sigma}$ (resp., $A_{\sigma}$ ) be the maximal split torus in the group of elements of $A$ which are invariant (resp., antiinvariant) under $\sigma$. Then $\mathfrak{a}^{\sigma}$ (resp., $\mathfrak{a}_{\sigma}$ ) is identified with the set $\mathfrak{a}^{\sigma}$ (resp., $\mathfrak{a}^{-\sigma}$ ) of invariant (resp., antiinvariant) elements of $\mathfrak{a}$ under $\sigma$ and $A_{\sigma}$ is the maximal $\sigma$-split torus of A. 
If $M$ is a $\sigma$-invariant Levi subgroup of $G$ which contains $A, \mathfrak{a}_{M}$ is a $\sigma$-invariant subspace of $\mathfrak{a}$ and $\mathfrak{a}_{M}=\mathfrak{a}_{M}^{\sigma} \oplus \mathfrak{a}_{M}^{-\sigma}$ where $\mathfrak{a}_{M}^{\sigma}=\mathfrak{a}_{M} \cap$ $\mathfrak{a}^{\sigma}, \mathfrak{a}_{M}^{-\sigma}=\mathfrak{a}_{M} \cap \mathfrak{a}^{-\sigma}$. This is an orthogonal decomposition and, in the identification of $\mathfrak{a}$ to $\mathfrak{a}^{\prime},\left(\mathfrak{a}_{M}\right)^{-\sigma}$ is identified with the space $\left(\mathfrak{a}_{M}^{\prime}\right)^{-\sigma}$ of $\sigma$-antiinvariant elements of $\mathfrak{a}_{M}^{\prime}$.

Moreover the Lie algebra of the connected component, $X(M)_{\sigma}$, of the group of antiinvariant elements of $X(M)$, with the identification of $\mathfrak{a}_{M}$ and $\mathfrak{a}_{M}^{\prime}$, is equal to $\mathfrak{a}_{M}^{-\sigma}$.

A parabolic subgroup of $G, P$, is called a $\sigma$-parabolic subgroup if $P$ and $\sigma(P)$ are opposite parabolic subgroups. Then $M:=P \cap \sigma(P)$ is

(2.19) the $\sigma$-stable Levi subgroup of $P$. If $P$ is such a parabolic subgroup, $P^{-}$will denote $\sigma(P)$. Then the maximal split torus of the center of $M, A_{M}$ is $\sigma$-stable.

The sentence "Let $P=M U$ be a parabolic subgroup of $G$ " will mean that $U$ is the unipotent radical of $P$ and $M$ is a Levi subgroup of $G$. If moreover $P$ is a $\sigma$-parabolic subgroup of $G, M$ will denote its $\sigma$-stable Levi subgroup.

One deduces from [18, Proposition 13.4]:

$P H$ and $P^{-} H$ are open in $G$. Let $M$ be equal to $P \cap \sigma(P)$. The restriction to $H \cap M$ of the modulus function of $P, \delta_{P}$, is trivial as it is positive and equal to its inverse on $M \cap H$.

We also recall that (cf. loc. cit. Corollary 6.16):

There are only a finite number of $H$-conjugacy classes of parabolic subgroups of $G$.

Let $M_{\emptyset}$ be the $\sigma$-stable Levi subgroup of a minimal $\sigma$-parabolic subgroup of $G$. Let $A_{\emptyset}$ be the maximal $\sigma$-split torus of the center of $M_{\emptyset}$.

Definition 2.1. An element $x$ of $G$ is said to be $A_{\emptyset}$-good if and only if $x^{-1} \cdot A_{\emptyset}$ is a $\sigma$-split torus.

From [3, Lemma 2.4] one sees:

There exists a finite set of representatives of the $\left(P_{\emptyset}, H\right)$-double cosets open in $G, \mathcal{W}_{M_{\emptyset}}^{G}$, whose elements are $A_{\emptyset}$-good.

Moreover if $M$ is the $\sigma$-stable Levi subgroup of a $\sigma$-parabolic subgroup of $G$, with $M_{\emptyset}$ (or $A_{\emptyset}$ ) contained in $M$, there exists a subset, $\mathcal{W}_{M}^{G}$, of $\mathcal{W}_{M_{\emptyset}}^{G}$ such that for all $\sigma$-parabolic subgroups $P$ of $G$ with Levi subgroup $M, \mathcal{W}_{M}^{G}$ is a set of representatives of the $(P, H)$-double open cosets.

Lemma 2.2. Let the notation be as above. Let $P=M U$ be a $\sigma$-parabolic subgroup of $G$ with $M_{\emptyset} \subset M$ and let $x \in G$ be $A_{\emptyset}$-good. Then one has:

(i) The group $x^{-1} . P$ is a $\sigma$-parabolic subgroup of $G$ with $\sigma$-stable Levi subgroup $x^{-1}$.M. Moreover $\sigma\left(x^{-1} \cdot P\right)=x^{-1} \cdot P^{-}$, where $P^{-}=\sigma(P)$.

(ii) The set $P x H$ is open in $G$.

(iii) One defines an involution of $G, \sigma_{x}$, by:

$$
\sigma_{x}(g):=x \sigma\left(x^{-1} g x\right) x^{-1}, g \in G,
$$

whose fixed point set contains $x . H$. Then $P$ is a $\sigma_{x}$-parabolic subgroup of $G$, $\sigma_{x}(M)=M$ and $\sigma_{x}(P)=P^{-}$. 
(iv) For all $y \in P x H, y^{-1} . P$ is a $\sigma$-parabolic subgroup of $G$ and $P$ is a $\sigma_{y^{-}}$ parabolic subgroup of $G$. Let $M_{y}$ be the $\sigma_{y}$-stable Levi subgroup of $P$. Then $M_{y} \cap$ $y . H=P \cap y . H$. Moreover if $y=p x$ with $p \in P$, one has $M_{y}=p . M$.

Proof. (i) and (ii) follow from [3, Lemma 2.4].

(iii) follows immediately from (i).

(iv) If $y=p x h$ with $h \in H, p \in P$, then $y^{-1} \cdot P=h^{-1} \cdot\left(x^{-1} \cdot P\right)$. Then the first part of (iv) follows from (i). One also has $\sigma_{y}=\sigma_{p x}$. Hence one is reduced to prove the second part of (iv) for $h=1$ and $y=p x$. A simple computation shows that $\sigma_{y}(P)=p . P^{-}$. Hence $\sigma_{y}(P) \cap P=p . M$, which proves (iv).

Let $A_{\emptyset}$ be a maximal $\sigma$-split torus of $G$ and let $A_{0}$ be a $\sigma$-stable maximal split torus of $G$ which contains $A_{\emptyset}$. Let $P_{\emptyset}=M_{\emptyset} U_{\emptyset}$ be a minimal $\sigma$-parabolic subgroup of $G$, whose $\sigma$-stable Levi subgroup is equal to the centralizer, $M_{\emptyset}$, of $A_{\emptyset}$ in $G$.

Proposition 2.3. There exists a decreasing sequence of $\sigma$-stable compact open subgroups of $G,\left(J_{n}\right)_{n \in \mathbb{N}^{*}}$, which forms a basis of neighborhoods of 1 in $G$ and such that for each $n \geq 1, J:=J_{n}$ satisfies:

(i) For every $\sigma$-parabolic subgroup of $G, P=M U$ which contains $P_{\emptyset}$, the product map $J_{U^{-}} \times J_{M} \times J_{U} \rightarrow J$ is bijective, where $J_{U^{-}}=J \cap U^{-}, J_{M}=J \cap M, J_{U}=J \cap U$.

(ii) Let $A \subset A_{\emptyset}$ be the maximal $\sigma$-split torus of the center of $M$ and let $A^{-}$be the set of its $P$-antidominant elements. For all a belonging to $A^{-}$, one has

$$
a J_{U} a^{-1} \subset J_{U}, a^{-1} J_{U^{-}} a \subset J_{U^{-}} .
$$

(iii) One has $J=J_{H} J_{P}$, where $J_{H}=J \cap H, J_{P}=J \cap P$.

(iv) For each $\sigma$-parabolic subgroup of $G$ which contains $P_{\emptyset}, P=M U$, the sequence $\left(J_{n} \cap M\right)$ enjoys the same properties as those of $\left(J_{n}\right)$ for $M, P_{\emptyset} \cap M$.

Remark 2.4. If the characteristic of the residue field of $\mathbf{F}$ is different from 2, the proposition is due to Katano and Takano (cf. [20, Lemma 4.3]). In that case, their result is stronger, as their $J_{n}$ satisfy more properties.

Proof. We use the terminology and notation of Section 10 (cf. also Lemma 10.2). We fix a basis of the Lie algebra of $G, g,\left(X_{j}\right)$, which is the union of a basis $\left(U_{k}\right)$ of $u_{\emptyset}$ made of weight vectors for $A_{\emptyset}$, of a basis of $m_{\emptyset} \cap \underline{h}$, a basis of the space of $\sigma$-antiinvariant elements of $\underline{m_{\emptyset}}$ and the basis $\left(\sigma\left(U_{k}\right)\right)$ of $\sigma\left(\underline{u_{\emptyset}}\right)$. We use Lemma 10.1 (iv) for $G_{1}=U, G_{2}=M, \overline{G_{3}}=\sigma(U)$ to prove (i).

Let $A^{0}$ be the maximal compact open subgroup of $A$. One can choose a finite family $\left(\lambda_{l}\right)$ such that the family $\left(A^{0} \lambda_{l}\right)$ generates the monoid $A^{-}$. We apply Lemma 10.1 (iii) to the family of automorphisms of $G$ induced by the conjugation by elements of $A^{0} \lambda_{l}$. The fact that $J_{n}$ can be chosen $\sigma$-stable is proven similarly.

(iii) We apply Lemma 10.1 to $G_{1}=U, G_{2}=M, G_{3}=H$. Here $\underline{g}_{1}^{\prime}=\underline{u}, \underline{g}_{2}^{\prime}=\underline{m}$ and $\underline{g}_{3}^{\prime}$ is the subspace generated by $U_{i}+\sigma\left(U_{i}\right)$, where $U_{i}$ are those which belong to $\underline{u}$.

(iv) The results from the fact that $J_{M}$ is defined like $J$, in view of Lemma 10.1 (iv).

Remark 2.5. In [12, Remark 3.2], it was stated incorrectly, although not used, that in (ii) one could replace $A$ by the maximal split torus of the center of $M$. 
If a compact open subgroup of $G$ satisfies the properties of Proposition

(2.23) $\quad 2.3$, it will be said to have a $\sigma$-factorization. These are the $(P, H)$-good subgroups from the introduction.

If $\left(P=M U, P^{-}=M U^{-}\right)$is a pair of opposite parabolic subgroups of $G$, we will say that a compact open subgroup $J$ of $G$ has an Iwahori factorization with respect to $\left(P, P^{-}\right)$if the product map $J_{U^{-}} \times J_{M} \times$ $J_{U} \rightarrow J$ is bijective, where $J_{U^{-}}=J \cap U^{-}, J_{M}=J \cap M, J_{U}=J \cap U$.

3. TWO OPERATIONS ON $H$-FORMS ON INDUCED REPRESENTATIONS

3.1. Second Adjointness Theorem and $H$-forms. In the sequel, the smooth representations of $G$ and of its closed subgroups will have complex coefficients.

Let $(\pi, V)$ be a smooth representation of $G$ and let $P=M U$ be a parabolic subgroup of $G$. One denotes by $\left(\pi_{P}, V_{P}\right)$ the tensor product of the quotient of $V$ by the $M$-submodule generated by $\pi(u) v-v, u \in U, v \in V$, with the representation of $M$ on $\mathbb{C}$ given by $\delta_{P}^{-1 / 2}$. We call it the normalized Jacquet module of $V$ along $P$. We denote the natural projection map from $V$ to $V_{P}$ by $j_{P}$ and sometimes $\pi_{P}$ will be denoted $j_{P}(\pi)$. For further reference, we state the following fact:

Let $P=M U$ be a parabolic subgroup of $G$, let $Q=L V$ be a parabolic subgroup of $M$ and let $(\delta, E)$ be a smooth representation of $M$. If $x$ is an element of $G$, one has the equality of vector spaces $(x E)_{x \cdot Q}=E_{Q}$, and the natural representation of $x . Q$ on $(x E)_{x . Q}$ is simply $x . \delta_{Q}$.

The following result is due to J. Bernstein for smooth representations (cf. 2], [25, Chapter V.9]; see also [7] for the first published proof). Here we present a slight reformulation of his result (cf. [12, Lemma 2.1]). This is a generalization of a result of W. Casselman for admissible representations (cf. [8]). Let $\left(P, P^{-}\right)$be a pair of opposite parabolic subgroups of $G$ with common Levi subgroup $M$. Let $A_{0}$ be a maximal split torus of $M$ and let $P_{0}$ be a minimal parabolic subgroup $P_{0}$ such that $A_{0} \subset P_{0} \subset P$. We define:

and, for $\varepsilon>0$, we set:

$$
\Theta_{P}:=\Delta\left(P_{0} \cap M, A_{0}\right)
$$

$$
A_{0}^{-}(P,<\varepsilon):=\left\{\left.a \in A_{0}^{-}|| \alpha(a)\right|_{\mathbf{F}}<\varepsilon, \alpha \in \Delta\left(P_{0}, A_{0}\right) \backslash \Theta_{P}\right\} .
$$

\section{Second Adjointness Theorem.}

Let $(\pi, V)$ be a smooth representation of $G$. Let $j_{P}$ (resp., $\left.j_{P^{-}}\right)$denote the canonical projection of $V$ (resp., of the smooth dual $\tilde{V}$ of $V$ ) onto $V_{P}$ (resp., $\left.(\check{V})_{P^{-}}\right)$.

Then there exists a unique nondegenerate $M$-invariant bilinear form $\langle., .\rangle_{P}$ on $(\check{V})_{P^{-}} \times V_{P}$ such that for all compact open subgroups, $J$, of $G$, there exists $\varepsilon_{J}<1$ such that:

$$
\delta_{P}^{1 / 2}(a)\left\langle j_{P^{-}}(\check{v}), \pi_{P}(a) j_{P}(v)\right\rangle_{P}=\langle\check{v}, \pi(a) v\rangle,
$$

for $a \in A_{0}^{-}\left(P,<\varepsilon_{J}\right), v \in V^{J}, \check{v} \in \check{V}^{J}$. It is part of the statement that $\varepsilon_{J}$ does not depend on $V$.

In particular there is a canonical isomorphism between $\left(V_{P}\right)^{\check{\nu}},(\check{V})_{P^{-}}$.

(3.4) An $H$-form on a smooth module of $G$ is an $H$-fixed linear form on $V$. 
One denotes by $e_{J}$ the normalized Haar measure on $J$ that we view as a compactly supported distribution on $G$. Using the same argument as in [23. Lemma 2], one sees:

Let $P=M U$ be a $\sigma$-parabolic subgroup of $G$. Let $A$ be the maximal split torus of the center of $M$. Let $A_{\sigma}$ be the maximal $\sigma$-split torus of the maximal split torus, $A$, of the center of $M$. Let $J$ be a compact open subgroup of $G$ with a $\sigma$-factorization with respect to $\left(P, P^{-}\right)$. Then for every smooth module $(\pi, V), \xi \in V^{\prime} H$ :

$$
\langle\xi, \pi(a) v\rangle=\left\langle e_{J} \xi, \pi(a) v\right\rangle, v \in V^{J}, a \in A^{-} \cap A_{\sigma},
$$

where $e_{J} \xi$ is the element of $\check{V}$ defined by:

$$
\left\langle e_{J} \xi, v\right\rangle=\left\langle\xi, \pi\left(e_{J}\right) v\right\rangle, v \in V .
$$

From our hypothesis on $J$, one sees that one can take $J^{\prime}=J$ in the proof of Lemma 2 of 23 .

The following result has been proved independently on one hand by Kato and Takano ([20, Proposition 6]) and on the other by Lagier ([23, Theorem 1 (ii)]) for admissible modules. Later, it was remarked (cf. [12]) that it works for general smooth modules. Let $A_{M}$, or simply $A$, be the maximal split torus of the center of $M$. For every smooth module $(\pi, V)$ of $G$ and $\xi$ an $H$-form on $V$, there exists a unique $M \cap H$-form $j_{P^{-}} \xi$ on $V_{P}$ such that for each compact open subgroup $J$ of $G$, there exists $\varepsilon_{J}^{\prime}>0$, such that $\varepsilon_{J}^{\prime} \leq \varepsilon_{J}$, depending only on $J$ and not on $V$ and $\xi$. Thus one has:

$$
\langle\xi, \pi(a) v\rangle=\delta_{P}^{1 / 2}(a)\left\langle j_{P^{-}} \xi, \pi_{P}(a) j_{P}(v)\right\rangle, v \in V^{J}, a \in A^{-}\left(\varepsilon_{J}^{\prime}\right) .
$$

From (3.5), one deduces from the above that if $J$ is a compact open subgroup of $G$ with a $\sigma$-factorization, one has

$$
\left\langle e_{J} \xi, \pi(a) v\right\rangle=\delta_{P}^{1 / 2}(a)\left\langle j_{P^{-}} \xi, \pi_{P}(a) j_{P}(v)\right\rangle_{P}, v \in V^{J}, a \in A_{\sigma} \cap A^{-}\left(\varepsilon_{J}^{\prime}\right) .
$$

From the Second Adjointness Theorem, one deduces from this that for $a \in A_{\sigma} \cap$ $A^{-}\left(\varepsilon_{J}^{\prime}\right), v \in V^{J}:$

$$
\left\langle j_{P^{-}} \xi, \pi_{P}(a) j_{P}(v)\right\rangle=\left\langle j_{P^{-}} e_{J} \xi, \pi_{P}(a) j_{P}(v)\right\rangle .
$$

If $\pi$ is admissible, the two sides of this equality are $A_{\sigma}$-finite functions on $A_{\sigma}$, hence they are equal. In particular, in $a=1$ one gets:

Let $J$ be a compact open subgroup of $G$ with a $\sigma$-factorization with respect to $\left(P, P^{-}\right)$. Then, if $\pi$ is admissible, one has

$$
\left\langle j_{P^{-}} \xi, v\right\rangle=\left\langle j_{P^{-}}\left(e_{J} \xi\right), v\right\rangle_{P}, v \in V_{P}^{J_{M}} .
$$

\subsection{Induced.}

Let $A$ be a maximal split torus of $G$. Let $K$ be a maximal compact open subgroup of $G$ which is the stabilizer of a special point of the apartment of the extended building of $G$. It fixes a choice of a left invariant measure on each algebraic closed subgroup of $G$ such that the measure of its intersection with $K$ is equal to 1 . It depends on $A$ and $K$.

Let $P=M U$ be a parabolic subgroup of $G$ with Levi subgroup $M$ containing $A$ and with unipotent radical $U$. Let $\chi$ be an element of $X(M)$. One denotes by $E_{\chi}$ the space of the representation $\delta_{\chi}:=\delta \otimes \chi$. Let $i_{P}^{G} E_{\chi}$ be the space of maps $v$ from $G$ to $E$, right invariant under a compact open subgroup of $G$ and such that 
$v(m u g)=\delta_{P}(m)^{1 / 2} \delta_{\chi}(m) f(g)$ for all $m \in M, u \in U, g \in G$. Let $i_{P}^{G} \delta_{\chi}$ be the representation of $G$ in $i_{P}^{G} E_{\chi}$ by right translations.

If $(\delta, E)$ is a smooth representation of $M$, one extends it to a representation of $P$ trivial on $U$, denoted in the same way. One denotes by $i_{P \cap K}^{K} E$ the space of maps $v$ from $K$ in $E$, which are right invariant under a compact open subgroup of $K$ and such that $v(p k)=\delta(p) v(k)$ for all $k \in K$ and $p \in P \cap K$. The restriction of functions to $K$ determines an isomorphism from $i_{P}^{G} E_{\chi}$ to $i_{P \cap K}^{K} E$. We will denote the representation of $G$ in $i_{P \cap K}^{K} E$ deduced from $i_{P}^{G} \delta_{\chi}$ "par transport de structure" by $\bar{i}_{P}^{G}\left(\delta_{\chi}\right)$. This representation will be called the compact realization of $i_{P}^{G} \delta_{\chi}$ in this space independent from $\chi$. If $v \in i_{P \cap K}^{K} E$, one denotes by $v_{\chi}$ the element of $i_{P}^{G} E_{\chi}$ whose restriction to $K$ is equal to $v$. If $\delta$ is unitary, one defines a scalar product on $i_{P \cap K}^{K} E$ by:

$$
\left(v, v^{\prime}\right)=\int_{K}\left(v(k), v^{\prime}(k)\right) d k, v, v^{\prime} \in i_{P \cap K}^{K} E .
$$

The representation $\bar{i}_{P}^{G} \delta_{\chi}$ is unitary for this scalar product when $\chi$ is unitary. Consequently, "par transport de structure", $i_{P}^{G} \delta_{\chi}$ is also unitary.

If $g \in G$, one chooses $u_{P}(g) \in U, m_{P}(g) \in M$ and $k_{P}(g) \in K$ such that $g=$ $u_{P}(g) m_{P}(g) k_{P}(g)$. Then $\delta_{P}\left(m_{P}(g)\right)$ does not depend on the choice of $m_{P}(g)$. Let $P^{-}=M U^{-}$be the opposite parabolic subgroup of $P$ with respect to $M$.

We will identify $i_{P}^{G} \check{E}$ with $\left(i_{P}^{G} E\right)^{\swarrow}$ by associating to $\check{v} \in i_{P}^{G} \check{E}$ the linear form $\phi$ on $i_{P}^{G} E$ defined by

$$
\langle\phi, v\rangle=\int_{K}\langle\check{v}(k), v(k)\rangle d k, v \in i_{P}^{G} E .
$$

Let $e$ be an element of $E$ and let $J$ be a compact open subgroup of $G$ such that $e$ is invariant under $J \cap P$ under $\delta$. One defines a map $v_{e, \delta}^{P, J}$ from $G$ to $E$ by:

$$
\begin{gathered}
v_{e, \delta}^{P, J}(p j)=\delta_{P}^{1 / 2}(p) \delta(p) e, j \in J, p \in P, \\
v_{e, \delta}^{P, J}(g)=0, g \notin P J,
\end{gathered}
$$

the definition making sense due to our hypothesis on $J$ and $e$. Notice that this hypothesis is satisfied if $J$ has an Iwahori factorization with respect to $\left(P, P^{-}\right)$ (resp., if $P$ is a $\sigma$-parabolic subgroup of $G$ and $J$ has a $\sigma$-factorization for $\left(P, P^{-}\right)$) and $e$ is $J_{M}$-invariant.

In all cases $v_{e, \delta}^{P, J}$ is invariant on the right by $J$ and defines an element of $i_{P}^{G} E$.

3.3. The operation $\check{r}_{M}$. Let $P=M U$ be a parabolic subgroup of $G$ such that $A \subset M$ and $(\delta, E)$ is a smooth representation of $M$. Let $\pi=i_{P}^{G} \delta$.

Then with our choice of Haar measures, the left invariant Haar measure $d_{l} p$ on $P$ satisfies:

$$
\int_{G} f(g) d g=\int_{P \times K} f(p k) d_{l} p d k, f \in C_{c}^{\infty}(G) .
$$

Let $d_{r} p=\delta_{P} d_{l} p$, which is a right invariant measure on $P$. One defines a linear map $M_{\delta, P}$ from $C_{c}^{\infty}(G) \otimes E$ to $i_{P}^{G} E$ by:

$$
\left(M_{\delta, P}(f)\right)(g)=\int_{P} \delta_{P}^{1 / 2}\left(p^{-1}\right) \delta\left(p^{-1}\right) f(p g) d_{r} p, f \in C_{c}^{\infty}(G) \otimes E, g \in G,
$$


where we have identified $C_{c}^{\infty}(G) \otimes E$ with $C_{c}^{\infty}(G, E)$. This map goes through the quotient to an isomorphism between $H_{0}\left(P, C_{c}^{\infty}(G) \otimes E\right)$ ) and $i_{P}^{G} E$ ([3. Prop. 1.13 (iv)]), where $H_{0}$ stands for the 0-homology.

Lemma 3.1. We recall our choice of a left invariant measure on $P$ (cf. (3.12)). $A$ linear form $\xi$ on $i_{P}^{G} E$ determines an E-distribution on $G, \tilde{\xi}$, which is defined by

$$
\tilde{\xi}(f)=\left\langle\xi, M_{\delta, P}(f)\right\rangle, f \in C_{c}^{\infty}(G) \otimes E .
$$

The distribution $\tilde{\xi}$ is P-covariant for the representation $\pi=\delta \otimes \delta_{P}^{-1 / 2}$ (cf. Section 9.1 for the definitions).

Proof. Here we denote the left regular representation of $G$ on $C^{\infty}(G) \otimes E$ by $\lambda$. The lemma follows from the obvious equality:

$$
\langle\tilde{\xi}, \lambda(p)(\pi(p) f)\rangle=\left\langle\xi, M_{\delta, P}(\lambda(p)(\pi(p) f))\right\rangle, f \in C_{c}^{\infty}(G) \otimes E
$$

and from the equality:

$$
M_{\delta, P}(\lambda(p)(\pi(p) f))=M_{\delta, P}(f), f \in C_{c}^{\infty}(G) \otimes E,
$$

that we are going to prove. Let $p_{0}$ be an element of $P$. Taking into account the equality $\delta_{P}\left(p_{0}\right)^{-1 / 2}$ with $\delta_{P}\left(p_{0}\right)^{1 / 2} \delta_{P}^{-1}\left(p_{0}\right)$, one has, for $g \in G$ :

$$
\left[M_{\delta, P}\left(\lambda\left(p_{0}\right)\left(\pi\left(p_{0}\right) f\right)\right)\right](g)=\int_{P} \delta_{P}^{1 / 2}\left(p^{-1} p_{0}\right) \delta\left(p^{-1} p_{0}\right) f\left(p_{0}^{-1} p g\right) \delta_{P}\left(p_{0}\right)^{-1} d_{r} p .
$$

Using the definition of $d_{r} p$, the change of variables $p^{\prime}=p_{0}^{-1} p$ leads to the required identity.

The support of E-distributions is given in Section 9.1. We define the support of $\xi$ as follows:

The support of $\xi$ is by definition the support of $\tilde{\xi}$. As $\tilde{\xi}$ is left $P$ covariant, one has:

$\operatorname{Supp}(\xi)$ is left $P$-invariant and is equal to the complement of the largest left $P$-invariant open subset of $G, O$, such that $\langle\xi, v\rangle=0$ if the support of $v \in i_{P}^{G} E$ is contained in $O$.

If moreover $\xi$ is invariant under the right action of $H$, then the same is true for the E-distribution $\tilde{\xi}$.

Let us assume that $P H$ is open in $G$ and that $\xi$ is right invariant under $H$. The group $P \times H$ acts on $P H$ by

$$
(p, h) g=p g h^{-1}, g \in P H, p \in P, h \in H .
$$

Hence $X=P H$ is a homogeneous space under $P \times H$ homeomorphic to $X=$ $(P \times H) / \operatorname{Diag}(P \cap H)$ by the map $(p, h) \rightarrow p h^{-1}($ cf. [3. Lemma 3.1 (iii)]). One remarks that the Haar measure on $G$ restricted to $P H$ is left invariant under $P$ and right invariant under $H$. One applies Lemma 9.1 to $P \times H($ instead of $G)$ acting on $P H$ and to the $\delta \otimes \delta_{P}^{-1 / 2}$-covariant distribution $\tilde{\xi}$ restricted to $P H$. From above, $X$ has a $P \times H$-invariant measure. The dual representation of $\delta_{P}$ is $\delta_{P}^{-1}$. Hence there exists a unique $H \cap P$-invariant linear form $\eta$ on $E$ such that one can define:

$$
f_{\xi}(g):=\left(\delta_{P}\right)(p)^{1 / 2} \delta^{\prime}(p) \eta, g=p h, p \in P, h \in H,
$$


which verifies:

$$
\begin{aligned}
& \langle\tilde{\xi}, f\rangle=\int_{P H}\left\langle f_{\xi}(g), f(g)\right\rangle d g \text { if } f \in C_{c}^{\infty}(G) \otimes E \text { has its support contained } \\
& \text { in } P H .
\end{aligned}
$$

Let us assume that moreover $f$ has its support contained in $P(K \cap H)$. The set of $(p, k) \in P \times K$ such that $p k \in P(K \cap H)$ is equal to $P \times(K \cap P)(K \cap H)$. Thus, from (3.12), one gets:

$$
\int_{P(K \cap H)}\left\langle f_{\xi}(g), f(g)\right\rangle d g=\int_{P \times(K \cap P)(K \cap H)}\left\langle f_{\xi}(p k), f(p k)\right\rangle d_{l} p d k .
$$

Then one integrates over $P$, taking into account the covariance property of $\xi$. Then, as $\delta_{P} d_{l} p=d_{r} p$, one gets:

$$
\langle\xi, v\rangle=\int_{(K \cap P)(K \cap H)}\left\langle f_{\xi}(k), v(k)\right\rangle d k,
$$

where $v=M_{\delta, P}(f)$.

One remarks that if $e$ is fixed by $J \cap P$, using the notation of (3.11), one has:

$$
v_{e, \delta}^{P, J}=\operatorname{Vol}(J \cap P)^{-1} M_{\delta, P}\left(1_{J} \otimes e\right),
$$

where $\operatorname{Vol}(J \cap P)$ denotes the volume of $J \cap P$ for the measure $d_{l} p$.

Let $x$ in $G$ be such that $P x H$ is open in $G$. Applying (3.16) and (3.17) to each $\pi^{\prime}(x) f$, which is $x H x^{-1}$-invariant, one can define a function on the union $\Omega$ of all $(P, H)$ open double cosets, $f_{\xi}$, with values in $E^{\prime}$, right invariant under $H$, and left covariant under $P$ by $\delta^{\prime} \otimes \delta_{P}^{-1 / 2}$, such that:

$$
\text { If } f \in C_{c}^{\infty}(G) \otimes E \text { has its support contained in } \Omega \text {, }
$$

$$
\langle\tilde{\xi}, f\rangle=\int_{\Omega}\left\langle f_{\xi}(g), f(g)\right\rangle d g
$$

Moreover if $x \in G$ is such that $P x H$ is open in $G$ :

$$
f_{\xi}(x) \in E^{P \cap x \cdot H} .
$$

Notice that $f_{\xi}$ depends on our choice of $d g$.

Let us assume moreover that $P$ is a $\sigma$-parabolic subgroup of $G$. Let $J$ be a compact open subgroup of $K$ which has a $\sigma$-factorization for $\left(P, P^{-}\right)$. Let $e \in E$ be fixed by $J_{M}$. Let us prove:

$$
\left\langle\xi, v_{e, \delta}^{P, J}\right\rangle=\operatorname{Vol}\left((K \cap P) J_{H}\right)\langle\eta, e\rangle .
$$

One can apply (3.18) with $v=v_{e, \delta}^{P, J}$ by taking into account (3.19). As the support of $v$ is contained $P J=P J_{H}$ where $J_{H}=J \cap H$, one gets from (3.18):

$$
\langle\xi, v\rangle=\int_{K \cap\left(P J_{H}\right)}\left\langle f_{\xi}(k), v(k)\right\rangle d k .
$$

But $K \cap\left(P J_{H}\right)=(K \cap P) J_{H}$. So, one has:

$$
\langle\xi, v\rangle=\int_{(K \cap P) J_{H}}\left\langle f_{\xi}(k), v(k)\right\rangle d k .
$$


The function under the integral sign is left invariant under $K \cap P$, due to (3.16) and to the properties of the induced representation. It is right invariant under $J_{H}$ due to the fact that $\xi$ is $H$-invariant and that $v$ is $J$-invariant. Hence (3.21) follows.

Let $\check{v}$ be a smooth (resp., $\xi$ be an $H$-fixed) linear form on $i_{P}^{G} E$, where $P=M U$ is a parabolic subgroup of $G$ (resp., a parabolic subgroup of $G$ such that $P H$ is open in $G)$ such that $A \subset M$ and $(\delta, E)$ is a smooth representation of $M$. We have identified $\check{v}$ with an element of $i_{P}^{G} \check{E}$ (cf $(3.10)$ ), and we will denote its value at 1 by $\check{r}_{M} \check{v} \in \check{E}$. Similarly $f_{\xi}(1) \in E^{\prime M \cap H}$ is well defined. We will denote it by $\check{r}_{M} \xi$.

Proposition 3.2. Let us assume that $A$ is a $\sigma$-stable maximal split torus which contains a maximal $\sigma$-split torus.

(i) If $P=M U$ is a $\sigma$-parabolic subgroup of $G$ such that $A \subset M$, for all compact open subgroups of $K, J$, with a $\sigma$-factorization with respect to $\left(P, P^{-}\right)$, one has:

$$
\check{r}_{M}\left(e_{J} \xi\right)=e_{J_{M}}\left(\check{r}_{M} \xi\right) .
$$

(ii) Let $Q=L V$ be a a parabolic subgroup of $G$, such that $Q H$ is open and let $P=M U$ be a parabolic subgroup, such that $P \subset Q$ and $A \subset M \subset L$. Let $(\delta, E)$, $\check{v}, \xi$ be as above. Applying induction in stages, $i_{P}^{G} E$ is isomorphic to $i_{Q}^{G}\left(i_{P \cap L}^{L} E\right)$. From (3.22), one gets an element $\check{r}_{L} \check{v}$ of $\left(i_{P \cap L}^{L} E\right)^{\sim}\left(\right.$ resp. $\check{r}_{L} \xi$ of $\left.\left(i_{P \cap L}^{L} E\right)^{\prime L \cap H}\right)$.

(iii) Let us assume that $P$ and $Q$ are $\sigma$-parabolic subgroups of $G$. Then:

$$
\check{r}_{M} \xi=\check{r}_{M}\left(\check{r}_{L} \xi\right) \text {. }
$$

Proof. (i) One reduces easily to compare the evaluation on any element $e$ of $E^{J_{M}}$ of both sides of the equality that we want to prove. One introduces $v:=v_{e, \delta}^{P, J}$. From (3.21), one gets, on one hand:

$$
\langle\xi, v\rangle=\operatorname{Vol}\left((K \cap P) J_{H}\right)\left\langle f_{\xi}(1), e\right\rangle .
$$

As $v$ is $J$-invariant, this implies:

$$
\left\langle e_{J} \xi, v\right\rangle=\operatorname{Vol}\left((K \cap P) J_{H}\right)\left\langle f_{\xi}(1), e\right\rangle .
$$

On the other hand:

$$
\left\langle e_{J} \xi, v\right\rangle=\int_{K}\left\langle\left(e_{J} \xi\right)(k), v(k)\right\rangle d k .
$$

Again, we use the fact that the support of $v$ is contained in $P J=P J_{H}$ :

$$
\left\langle e_{J} \xi, v\right\rangle=\int_{(K \cap P) J_{H}}\left\langle\left(e_{J} \xi\right)(k), v(k)\right\rangle d k .
$$

The function under the integral sign is left invariant under $K \cap P$, by the properties of the induced representations, and right invariant under $J_{H}$ as $v$ and $e_{J} \xi$ are invariant under $J$. So one gets:

$$
\left\langle e_{J} \xi, v\right\rangle=\operatorname{Vol}\left((K \cap P) J_{H}\right)\left\langle\left(e_{J} \xi\right)(1), e\right\rangle .
$$

The equality needed to prove that (i) follows from this and from (3.23).

(ii) is a simple consequence of (i).

(iii) It is easy to see that for all $\check{v} \in\left(i_{P}^{G} E\right)^{\sim}$ the equality $\check{r}_{M} \check{v}=\check{r}_{M}\left(\check{r}_{L} \check{v}\right)$ holds. Then (iii) follows from the last assertion of (i). 
3.4. The operation $\check{j}_{Q^{-}}$. . Our second operation needs some preparation.

Let $P=M U$ be a parabolic subgroup of $G$. Let $\left(Q, Q^{-}\right)$be a pair of opposite parabolic subgroups of $M$ with $L:=Q \cap Q^{-}$. Let us assume that $A \subset L \subset M$. Let $(\delta, E)$ be a smooth representation of $M$. We denote the parabolic subgroup of $G$ equal to $Q U$ (resp., $Q^{-} U$ ) by $P_{Q}$ (resp., $P_{Q^{-}}$). We define a $G$-homomorphism $\breve{j}_{Q^{-}}$from $\left(i_{P}^{G} E\right)^{\llcorner}$to $\left(i_{P_{Q^{-}}}^{G} E_{Q}\right)^{\sim}$ as follows. Let $\check{v} \in\left(i_{P}^{G} E\right)^{\llcorner}$. As in (3.10) we identify $\left(i_{P}^{G} E\right)^{\swarrow}$ with $i_{P}^{G} \check{E}$ and we denote the element of $\left.i_{P_{Q^{-}}}^{G}\left((\check{E})_{Q^{-}}\right)\right)$obtained by the composition of $\check{v}$ with the projection $j_{Q^{-}}$by $j_{Q^{-}} \circ \check{v}$. By the Second Adjointness Theorem (cf. $(3.3),(\check{E})_{Q^{-}}$is canonically isomorphic to $\left(E_{Q}\right)^{2}$. Let us denote the image of $j_{Q^{-}} \circ \check{v}$

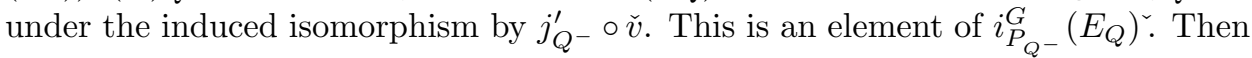
one defines $\check{j}_{Q^{-}} \circ \check{v}$ as the image of $j_{Q^{-}}^{\prime} \circ \check{v}$ under the isomorphism defined by (3.10) of $i_{P_{Q^{-}}}^{G}\left(E_{Q}\right)^{\llcorner}$with $\left(i_{P_{Q^{-}}}^{G} E_{Q}\right)^{\llcorner}$. Summarizing, $\check{j}_{Q^{-}}$. appears as the composition of the homomorphisms of $G$-modules:

$$
\left(i_{P}^{G} E\right)^{\llcorner} \rightarrow i_{P}^{G} \check{E} \stackrel{f}{\rightarrow} i_{P_{Q^{-}}}^{G}\left((\check{E})_{Q^{-}}\right) \stackrel{g}{\rightarrow} i_{P_{Q^{-}}^{G}}^{G}\left(E_{Q}\right)^{\llcorner} \rightarrow\left(i_{P_{Q^{-}}}^{G} E_{Q}\right)^{\llcorner}
$$

where the first arrow is the isomorphism (3.10) between $\left(i_{P}^{G} E\right)^{-}$and $i_{P}^{G} \check{E}$, the last arrow is the isomorphim (3.10) between $i_{P_{Q^{-}}}^{G}\left(E_{Q}\right)^{2}$ and $\left(i_{P_{Q^{-}}}^{G} E_{Q}\right)^{2}$, the arrow $f$ is given by composition of functions with the projection $j_{Q^{-}}$, and the arrow $g$ is the induced morphism from the canonical isomorphism between $(\check{E})_{Q^{-}}$and $\left(E_{Q}\right)^{-}$given by the Second Adjointness Theorem. Hence $\breve{j}_{Q^{-}} \circ$ is a $G$-module homomorphism. One sees easily that:

For $\check{v} \in\left(i_{P}^{G} E\right) \check{\text {, one has: }}$

$$
\left(\check{j}_{Q^{-}} \circ \check{v}\right)(1)=j_{Q^{-}}(\check{v}(1)),
$$

where on the left hand side of the equality $\breve{j}_{Q^{-}} \circ \check{v}$ is viewed as an element of $i_{P_{Q^{-}}}^{G}\left(E_{Q}\right)^{r}$ and where on the right side $\check{v}$ is viewed as an element of $i_{P}^{G} \check{E}$ (cf. (3.10) ) and $j_{Q^{-}}(\check{v}(1))$ is viewed as an element of $\left(E_{Q}\right)^{`}$ by the Second Adjointness Theorem. In other words:

$$
\check{r}_{L}\left(\check{j}_{Q^{-}} \circ \check{v}\right)=j_{Q^{-}}\left(\check{r}_{M}(\check{v})\right) \text {. }
$$

Proposition 3.3. Let $P=M U$ be a parabolic subgroup of $G$. Let $\left(Q, Q^{-}\right)$be a pair of opposite parabolic subgroups of $M$. Let us assume that $A \subset L:=Q \cap Q^{-}$. Let $(\delta, E)$ be a smooth representation of $M$ and let $\xi$ be an $H$-form on $V=i_{P}^{G} E$.

(i) Let $v_{1} \in V_{1}:=i_{P_{Q^{-}}}^{G} E_{Q}$. The number $\left\langle\check{j}_{Q^{-}} \circ\left(e_{J} \xi\right), v_{1}\right\rangle$ does not depend on the compact open subgroup $J$ of $G$ such that $v_{1}$ is fixed by $J$.

(ii) This allows us to define a linear form on $V_{1}$, denoted by $\check{j}_{Q^{-}} \circ \xi$, as follows.

If $v_{1} \in V_{1}$ is fixed by the compact open subgroup $J$ of $G$, one defines

$$
\left\langle\check{j}_{Q^{-}} \circ \xi, v_{1}\right\rangle:=\left\langle\check{j}_{Q^{-}} \circ\left(e_{J} \xi\right), v_{1}\right\rangle .
$$

Then $\check{j}_{Q^{-}} \circ \xi$ is an $H$-form on $V_{1}$.

(iii) For every compact open subgroup $J$ of $G$, one has:

$$
e_{J}\left(\check{j}_{Q^{-}} \circ \xi\right)=\check{j}_{Q^{-}} \circ\left(e_{J} \xi\right) \text {. }
$$

(iv) The support of $\check{j}_{Q^{-}} \circ \xi$ is contained in the support of $\xi$. 
Proof. (i) Let $v_{1} \in V_{1}$. It is enough to prove that if $J^{\prime} \subset J$ are two compact open subgroups of $G$ which leave $v_{1}$ invariant, one has:

$$
\left\langle\check{j}_{Q^{-}} \circ\left(e_{J^{\prime}} \xi\right), v_{1}\right\rangle=\left\langle\check{j}_{Q^{-}} \circ\left(e_{J} \xi\right), v_{1}\right\rangle \text {. }
$$

As $e_{J} v_{1}=v_{1}$, one has:

$$
\begin{gathered}
\left\langle\check{j}_{Q^{-}} \circ\left(e_{J^{\prime}} \xi\right), v_{1}\right\rangle=\left\langle\check{j}_{Q^{-}} \circ\left(e_{J^{\prime}} \xi\right), e_{J} v_{1}\right\rangle=\left\langle e_{J} \check{j}_{Q^{-}} \circ\left(e_{J^{\prime}} \xi\right), v_{1}\right\rangle \\
=\left\langle\check{j}_{Q^{-}} \circ\left(e_{J} \xi\right), v_{1}\right\rangle,
\end{gathered}
$$

which proves (i).

(ii) Let $\pi$ (resp., $\pi_{1}$ ) be the induced representation of $G$ on $V=i_{P}^{G} E$ (resp., $\left.V_{1}=i_{P_{Q^{-}}}^{G} E_{Q}\right)$. Let $v_{1}$ be an element of $V_{1}$ and let $h$ be an element of $H$. One may choose a small enough compact open subgroup $J$ of $G$, such that h.J and $h^{-1} . J$ also leave $v_{1}$ under $\pi_{1}$ invariant. This implies that $\pi_{1}(h) v_{1}$ is also fixed by $J$. Then, one has, from the definition of $\check{j}_{Q^{-}} \circ \xi$ :

$$
\left\langle\check{j}_{Q^{-}} \circ \xi, \pi_{1}(h) v_{1}\right\rangle=\left\langle\check{j}_{Q^{-}} \circ\left(e_{J} \xi\right), \pi_{1}(h) v_{1}\right\rangle .
$$

By elementary operations one sees that

$$
\begin{aligned}
\left\langle\check{j}_{Q^{-}} \circ \xi, \pi_{1}(h) v_{1}\right\rangle=\left\langle\check{j}_{Q^{-}} \circ\right. & \left.\left(\pi^{\prime}\left(h^{-1}\right) e_{J} \xi\right), v_{1}\right\rangle=\left\langle\check{j}_{Q^{-}} \circ\left(\pi^{\prime}\left(h^{-1}\right) e_{J} \pi^{\prime}(h) \xi\right), v_{1}\right\rangle \\
& =\left\langle\check{j}_{Q^{-}} \circ\left(e_{h^{-1} . J} \xi\right), v_{1}\right\rangle .
\end{aligned}
$$

Hence one gets, from the definition of $\check{j}_{Q^{-}} \circ \xi$ and the fact that $v_{1}$ is $h^{-1} . J$ fixed, the equality:

$$
\left\langle\check{j}_{Q^{-}} \circ \xi, \pi_{1}(h) v_{1}\right\rangle=\left\langle\check{j}_{Q^{-}} \circ \xi, v_{1}\right\rangle,
$$

which proves the $H$-invariance of $\check{j}_{Q^{-}} \circ \xi$.

The linearity is proved in the same way. This proves (ii).

(iii) is an immediate corollary of (ii).

(iv) Let $F$ be the support of $\xi$ and let $v_{1} \in V_{1}$ whose support, $F_{1}$, is contained in the complement of $F$ in $G$. Let us choose a compact open subgroup $J$ of $G$ which fixes $v_{1}$ and such that $F J$ is disjoint from $F_{1}$, which might be achieved from the compactness of $P_{Q} \backslash G$.

Then the support of $e_{J} \xi$ is disjoint from the support of $v_{1}$. As the composition by $j_{Q^{-}}$does not increase support, one concludes, from the definition in (ii), that:

$$
\left\langle\check{j}_{Q^{-}} \circ \xi, v_{1}\right\rangle=0 \text {. }
$$

This implies (iv).

Lemma 3.4. We keep the notation of the preceding proposition, but we assume that $P$ is a $\sigma$-parabolic subgroup with a $\sigma$-stable Levi subgroup $M$ and that $Q$ is a $\sigma$-parabolic subgroup of $M$ with a $\sigma$-stable Levi subgroup $L$. We assume moreover that $A$ is $\sigma$-stable, contained in $L$, and that it contains a maximal $\sigma$-split torus $A_{\emptyset}$ of $L$. Let $x$ be an element of $G$ which is $A_{\emptyset}$-good. Then $\pi^{\prime}(x) \xi$ is an $x . H$-form, $P$, $Q$ are $\sigma_{x}$-parabolic subgroups (see Lemma 2.2) and one has:

$$
\check{r}_{L}\left(\pi_{1}^{\prime}(x)\left(\check{j}_{Q^{-}} \circ \xi\right)\right)=j_{Q^{-}}\left(\check{r}_{M}\left(\pi^{\prime}(x) \xi\right)\right) .
$$

Proof. We first treat the case where $x=1$.

Notice that $\sigma\left(P_{Q^{-}}\right)=\left(P^{-}\right)_{Q}$ is opposite to $P_{Q^{-}}$. Hence $P_{Q^{-}}$is a $\sigma$-parabolic subgroup of $G$.

Let $e$ be an element of $E_{Q}$. From Proposition 2.3, one can choose a compact open subgroup of $K, J$, arbitrarily small, which has a $\sigma$-factorization with respect 
to $\left(P, P^{-}\right)$and $\left(P_{Q^{-}},\left(P^{-}\right)_{Q}\right)$, such that $J_{M}$ has a $\sigma$-factorization with respect to $\left(Q, Q^{-}\right)$. Hence we can choose $J$ such that $J_{L}$ fixes $e$. One has:

$$
\begin{gathered}
J=\left(J \cap P_{Q^{-}}\right)(J \cap H), \\
J_{M}=\left(J_{M} \cap Q^{-}\right)\left(J_{M} \cap H\right) .
\end{gathered}
$$

Let us prove

$$
\left\langle\check{r}_{L}\left(e_{J}\left(\check{j}_{Q^{-}} \circ \xi\right)\right), e\right\rangle=\left\langle\check{r}_{L}\left(\check{j}_{Q^{-}} \circ \xi\right), e\right\rangle .
$$

Let $P^{\prime}$ denote $P_{Q^{-}}$. As $e$ is fixed by $J_{L}, v:=v_{e, j_{Q}(\delta)}^{P^{\prime}, J}$ is well defined.

One will compute $\left\langle e_{J} \xi^{\prime}, v\right\rangle$ in two ways, where $\xi^{\prime}=\check{j}_{Q^{-}} \circ \xi$. First $v$ is invariant under $J$ so that one has:

$$
\left\langle e_{J} \xi^{\prime}, v\right\rangle=\left\langle\xi^{\prime}, v\right\rangle
$$

Using (3.18) and (3.19), one deduces from the preceding equality, as in the proof of (3.21), that:

$$
\left\langle e_{J} \xi^{\prime}, v\right\rangle=\int_{\left(P^{\prime} \cap K\right) J_{H}}\left\langle f_{\xi^{\prime}}(k), v(k)\right\rangle d k .
$$

The function under the integral sign is left invariant under $K \cap P^{\prime}$. Moreover, if $j \in J_{H}, f_{\xi^{\prime}}(j)=f_{\xi^{\prime}}(1)$ by the right $H$-invariance of $f_{\xi^{\prime}}$ and $v(j)=e$ by the right invariance by $J$ of $v$. So one gets:

$$
\left\langle e_{J} \xi^{\prime}, v\right\rangle=\operatorname{Vol}\left(\left(P^{\prime} \cap K\right) J_{H}\right)\left\langle f_{\xi^{\prime}}(1), e\right\rangle .
$$

Our second computation of $\left\langle e_{J} \xi^{\prime}, v\right\rangle$ starts with:

$$
\left\langle e_{J} \xi^{\prime}, v\right\rangle=\int_{K}\left\langle\left(e_{J} \xi^{\prime}\right)(k), v(k)\right\rangle d k .
$$

As $v$ is supported on $P^{\prime} J$, one gets:

$$
\left\langle e_{J} \xi^{\prime}, v\right\rangle=\int_{\left(K \cap P^{\prime}\right) J}\left\langle\left(e_{J} \xi^{\prime}\right)(k), v(k)\right\rangle d k
$$

As the function to integrate is invariant under $P^{\prime} \cap K$ on the left and by $J$ on the right, one has

$$
\left\langle e_{J} \xi^{\prime}, v\right\rangle=\operatorname{Vol}\left(\left(P^{\prime} \cap K\right) J\right)\left\langle\left(e_{J} \xi^{\prime}\right)(1), e\right\rangle
$$

Notice that:

$$
f_{\xi^{\prime}}(1)=\check{r}_{L}\left(\check{j}_{Q^{-}} \circ \xi\right),\left(e_{J} \xi^{\prime}\right)(1)=\check{r}_{L}\left(\left(e_{J} \xi^{\prime}\right)\right) .
$$

Then, taking into account the equality $J=\left(J \cap P^{\prime}\right) J_{H}$, one sees that $\left(P^{\prime} \cap K\right) J=$ $\left(P^{\prime} \cap K\right) J_{H}$. Then (3.26) follows from these two computations of $\left\langle e_{J} \xi^{\prime}, v\right\rangle$ (cf. (3.27) and (3.28) $)$.

From the fact that the composition by $j_{Q^{-}}$commutes with right translations by elements of $G$, one sees:

$$
e_{J}\left(\check{j}_{Q^{-}} \circ \xi\right)=\check{j}_{Q^{-}} \circ\left(e_{J} \xi\right)
$$

hence:

$$
\left\langle\check{r}_{L}\left(e_{J}\left(\check{j}_{Q^{-}} \circ \xi\right)\right), e\right\rangle=\left\langle\left(\check{r}_{L}\left(\check{j}_{Q^{-}} \circ\left(e_{J} \xi\right)\right), e\right\rangle .\right.
$$

From this and (3.24), one deduces:

$$
\left\langle\check{r}_{L}\left(e_{J}\left(\check{j}_{Q^{-}} \circ \xi\right)\right), e\right\rangle=\left\langle j_{Q^{-}}\left(\check{r}_{M}\left(e_{J} \xi\right)\right), e\right\rangle .
$$

As $J$ has a $\sigma$-factorization for $\left(P, P^{-}\right)$, one deduces from Proposition 3.2 (i), that

$$
\check{r}_{M}\left(e_{J}(\xi)\right)=e_{J_{M}} \check{r}_{M}(\xi) .
$$


Replacing it in the above equality, one gets:

$$
\left\langle\check{r}_{M}\left(e_{J}\left(\check{j}_{Q^{-}} \circ \xi\right)\right), e\right\rangle=\left\langle j_{Q^{-}}\left(e_{J_{M}} \check{r}_{M} \xi\right), e\right\rangle .
$$

From (3.7), and using the fact that $J_{M}$ has a $\sigma$-factorization for $\left(Q, Q^{-}\right)$, this implies:

$$
\left\langle\check{r}_{M}\left(e_{J}\left(\check{j}_{Q^{-}} \circ \xi\right)\right), e\right\rangle=\left\langle j_{Q^{-}}\left(\check{r}_{M} \xi\right), e\right\rangle .
$$

Together with (3.26), this shows that:

$$
\left\langle\check{r}_{L}\left(\check{j}_{Q^{-}} \circ \xi\right), e\right\rangle=\left\langle j_{Q^{-}}\left(\check{r}_{M} \xi\right), e\right\rangle
$$

which proves the assertion for $x=1$.

Let us treat the general case. Then (see Lemma 2.2), $P$ is a $\sigma_{x}$-parabolic subgroup of $G, M$ is $\sigma_{x}$-stable and $Q$ is a $\sigma_{x}$-parabolic subgroup of $M$. One may apply the first part of the proof to $\xi_{x}:=\pi^{\prime}(x) \xi$ which is fixed by $x . H$. The result follows from the fact that $\breve{j}_{Q^{-}}$○ is a $G$-module homomorphism.

\section{Generic Basic Geometric Lemma}

In the next two subsections, we make no assumptions on the characteristic of the residue field of $\mathbf{F}$.

4.1. Intertwining integrals. We keep the choices of (3.8). Let $P=M U$ be a parabolic subgroup of $G$ such that $A \subset M$, and let $(\delta, E)$ be a smooth representation of $M$ with finite length. Let $Q=M V$ be another parabolic subgroup of $G$ with $M$ as a Levi subgroup. We denote the set of roots of $A_{M}$ in the Lie algebra of $Q$ which are not roots of $A_{M}$ in the Lie algebra of $P$ by $\Sigma(Q, P)$. Let $P^{-}=M U^{-}$ be the opposite parabolic to $P$ which admits $M$ as a Levi subgroup. We have a right $V$-invariant measure on $V \cap U \backslash V$ which follows from our choice of measures (cf. (3.8)). We have a canonical identification of $V \cap U \backslash V$ with $V \cap U^{-}$.

We have $A_{M} \subset A$ and we fix a scalar product on $\mathfrak{a}^{\prime}$ which is invariant under the Weyl group of the pair $(G, A)$. It induces a scalar product on $\mathfrak{a}_{M}^{\prime}$ (cf. (2.13)).

One has (cf. [29, Theorem IV.1.1 and Proposition IV.2.1]):

There exists $R>0$ such that, for all $\chi \in X(M)$ which satisfies

$$
(\operatorname{Re}(\chi), \alpha)>R, \alpha \in \Sigma(Q, P),
$$

there exists an intertwining operator, $A\left(Q, P, \delta_{\chi}\right)$, between $i_{P}^{G} \delta_{\chi}$ and $i_{Q}^{G} \delta_{\chi}$ satisfying:

$$
\left(A\left(Q, P, \delta_{\chi}\right) f\right)(g)=\int_{V \cap U \backslash V} f(v g) d v, f \in i_{P}^{G} E_{\chi},
$$

the integral being absolutely convergent. This family of operators viewed in the compact realization admits an extension to a rational family in $\chi \in X(M)$ denoted in the same way. More precisely, there is a nonzero polynomial function $b$ on $X(M)$ such that for all $f$ in $i_{K \cap P}^{K} E$, the family $b(\chi)\left(A\left(Q, P, \delta_{\chi}\right) f\right)$ is polynomial, in the compact realization. 
From this characterization, one deduces:

The intertwining integrals commute with induced operators from intertwining operators between smooth representations of finite length of $M$. Namely let $\left(\delta^{1}, E^{1}\right)$ be another smooth admissible representation of $M$, and let us assume that $T$ is an intertwining operator between the representations $(\delta, E)$ and $\left(\delta_{1}, E_{1}\right)$. For $\chi \in X(M)$, the composition with $T$ determines an induced map $T_{\chi}^{P}$ between $i_{P}^{G} E_{\chi}$ and $i_{P}^{G} E_{\chi}^{1}$. With the notation above, one has:

$$
T_{\chi}^{Q} \circ A\left(Q, P, \delta_{\chi}\right)=A\left(Q, P, \delta_{\chi}^{1}\right) \circ T_{\chi}^{P} .
$$

Let us show that:

(4.3) When $A\left(Q, P, \delta_{\chi}\right)$ is defined, this operator is nonzero.

Let $P^{-}$be the opposite parabolic subgroup to $P$ with Levi subgroup $M$. Let $e \in E$ and let $J$ be a compact open subgroup with Iwahori factorization with respect to $\left(P, P^{-}\right)$and such that $e$ is invariant under $J_{M}$. Let $f=v_{e, \delta_{\chi}}^{P, J}$ whose support is $P J$. As $\left(V \cap U^{-}\right) \cap P J=V \cap\left(U^{-} \cap J\right)$ one sees that

$$
\left(A\left(Q, P, \delta_{\chi}\right) f\right)(1)=\operatorname{Vol}\left(V \cap U^{-} \cap J\right) e,
$$

which proves our claim.

The following lemma is an immediate consequence of the induction in stages and of the definitions.

Lemma 4.1. Let $P=M U$ be a parabolic subgroup of $G$ and let $Q_{1}=L V_{1}, Q_{2}=$ $L V_{2}$ be two parabolic subgroups of $M$. Let us assume $A \subset L$. Let us define $P_{Q_{1}}:=$ $Q_{1} U, P_{Q_{2}}:=Q_{2} U$. Let $(\omega, F)$ be a finite length smooth representation of $L$. By induction in stages, the representation $i_{P_{Q_{i}}}^{G} \omega$ is identified with $i_{P}^{G}\left(i_{Q_{i}}^{M} \omega\right)$.

Let $v \in i_{P_{Q_{1}}}^{G} E_{1}$. Then, with the identifications defined above, one has the equality of rational functions in $\chi \in X(L)$ :

$$
A\left(Q_{1}, Q_{2}, \omega_{\chi}\right) \circ v=A\left(P_{Q_{1}}, P_{Q_{2}}, \omega_{\chi}\right) v .
$$

From [29, IV.1 (11)], one has the relation:

$$
\langle A(Q, P, \delta) f, \check{f}\rangle=\langle f, A(P, Q, \check{\delta}) \check{f}\rangle, f \in i_{P}^{G} E, \check{f} \in i_{P}^{G} \check{E} .
$$

Let us prove:

Lemma 4.2. Let $\chi \in X(M)$ such that $A\left(Q, P, \delta_{\chi}\right)$ and $A\left(P, Q, \check{\delta_{\chi}}\right)$ are defined.

(i) Let $f \in i_{P}^{G} E_{\chi}$. Then one has:

$$
\operatorname{Supp}\left(A\left(Q, P, \delta_{\chi}\right) f\right) \subset \operatorname{cl}(V(\operatorname{Supp}(f))),
$$

where cl denotes the closure in $G$ and Supp the support.

(ii) Let $T$ be a linear form on $i_{Q}^{G} E_{\chi}$. Let $T^{\prime}=T \circ A\left(Q, P, \delta_{\chi}\right)$. Then one has:

$$
\operatorname{Supp}\left(T^{\prime}\right) \subset \operatorname{cl}(U(\operatorname{Supp}(T))) .
$$

Proof. (i) Let $g \notin \operatorname{cl}(V(\operatorname{Supp}(f)))$. Let us show that $g$ is not an element of the support of $\left(A\left(Q, P, \delta_{\chi}\right) f\right)$. One reduces immediately, by holomorphy, to the case where $A\left(Q, P, \delta_{\chi}\right)$ is defined by a converging integral. If there exists $v \in V$ such that $f(v g)$ does not vanish, $g$ has to be an element of $V \operatorname{Supp}(f)$. As this is not true, this implies that $\left(A\left(Q, P, \delta_{\chi}\right) f\right)(g)=0$. This proves (i). 
(ii) Let $A:=A\left(Q, P, \delta_{\chi}\right)$. Let $g$ be an element of $\operatorname{Supp}\left(T^{\prime}\right)$. Then for any compact open neighborhood $\Omega$ of $g$ in $G$, there exists an $f \in i_{P}^{G} E_{\chi}$ with support in $P \Omega$, such that $\left\langle T^{\prime}, f\right\rangle \neq 0$. Then $\langle T, A f\rangle \neq 0$, so that $\operatorname{Supp} T \cap \operatorname{Supp}(A f)$ is nonempty. By (i), $\operatorname{Supp}(A f) \subset c l(V \operatorname{Supp}(f))$. So one has:

$$
\operatorname{cl}(V P \Omega) \cap \operatorname{Supp}(T) \neq \emptyset .
$$

Let us show that if $X$ is a subset of $G$ and $\left(\Omega_{p}\right)$ is a decreasing sequence of compact open neighborhoods of $g$ in $G$, whose intersection is reduced to $g$,

$$
\bigcap_{p \in \mathbb{N}} c l\left(X \Omega_{p}\right)=\operatorname{cl}(X g) .
$$

In order to see this, one can reduce to the case where $g=e$. If $y \in \bigcap_{p \in \mathbb{N}} \operatorname{cl}\left(X \Omega_{p}\right)$, for all $p, y=\lim x_{n, p} \omega_{n, p}$, where $\omega_{n, p} \in \Omega_{p}$ and $x_{n, p} \in X$. Let $V, V^{\prime}$ be symmetric neighborhoods of $e$ in $G$ with $V^{\prime 2} \subset V$. Let $p \in \mathbb{N}$ such that $\Omega_{p} \subset V^{\prime}$ and let $n \in \mathbb{N}$ such that $y^{-1} x_{n, p} \omega_{n, p} \in V^{\prime}$. Then $y^{-1} x_{n, p}$ is an element of $V$. Hence $y$ is an element of $c l(X)$, which proves our claim. But, as $V P=Q U, V P \Omega_{p}$ is left $Q$-invariant and the image of $c l\left(V P \Omega_{p}\right)$ in $Q \backslash G$ is closed as the projection is open. Hence this projection is compact. It is the same for the projection of $\operatorname{Supp}(T)$. Together with (4.5), an argument of compactness shows that the intersection $\bigcap_{p \in \mathbb{N}} \operatorname{cl}\left(V P \Omega_{p}\right) \cap$ $\operatorname{Supp}(T)$ is nonempty. Together with (4.6), this implies:

$$
c l(V P g) \cap \operatorname{Supp}(T) \neq \emptyset .
$$

Then, one sees that $g \in \operatorname{cl}(P V \operatorname{Supp}(T))$ : if $\left(v_{n} p_{n} g\right)$ admits $t \in \operatorname{Supp}(T)$ as a limit, then $\left(v_{n} p_{n}\right)$ has $t g^{-1}$ as a limit and $\left(\left(v_{n} p_{n}\right)^{-1} t\right)$ has $g$ as a limit.

But, by the $Q$-invariance of $\operatorname{Supp}(T)$ and the equality $P V=U Q$, one has:

$$
P V \operatorname{Supp}(T)=U \operatorname{Supp}(T) .
$$

Hence $g$ is an element of $c l(U \operatorname{Supp}(T))$, which proves (ii).

Definition 4.3. A $(Q, P)$-subset of $X(M)$ is the complement in $X(M)$ of a finite union of sets of the form $\left\{\chi_{\nu} \mid \nu \in\left(\mathfrak{a}_{M}^{\prime}\right)_{\mathbb{C}},(\nu, \alpha)=c\right\}$, where $\alpha$ describes the set $\Sigma(Q, P)$.

It is clear that such a set is open and dense in $X(M)$.

Lemma 4.4. There exists a $(Q, P)$-subset of $X(M)$, such that for the $\chi$ element of this set, $A\left(Q, P, \delta_{\chi}\right)$ is invertible and the map $\chi \mapsto A\left(Q, P, \delta_{\chi}\right)$, viewed in the compact realization, is holomorphic on this set.

Proof. From [29, IV.1.1(12) and (14)], it suffices to prove the statement assuming that $P$ and $Q$ are adjacent and opposite, hence maximal. Let $\alpha$ be the single element of $\Sigma(Q, P)$. Recall that $\mathfrak{a}_{M}=\mathfrak{a}_{M}^{G} \oplus \mathfrak{a}_{G}(\mathrm{cf}$. (2.10) $)$. Here $\mathfrak{a}_{M}^{G}$ is one dimensional. Hence the image in $X(M)$ of $\left(\mathfrak{a}_{M}^{G}\right)_{\mathbb{C}}^{\prime}, X(M)^{G}$, under the map $\lambda \mapsto \chi_{\lambda}$, is a one dimensional torus. Thus the family $\chi \rightarrow A\left(Q, P, \delta_{\chi}\right)$, depending rationally on $\chi \in X(M)^{G}$, has a finite number of poles $\chi_{i}$. One remarks that $A\left(Q, P, \delta_{\chi}\right)$ does not change if $\chi$ is multiplied by an element of $X(G \mid M)$, so that $A\left(Q, P, \delta_{\chi}\right)$ has poles only along the sets $\chi_{i} X(G \mid M)$. This implies the holomorphy statement.

From [29, IV.3], there exists a rational function on $X(M)$ with values in $\mathbb{C}, j$, such that $A\left(P, Q, \delta_{\chi}\right) A\left(Q, P, \delta_{\chi}\right)$ is equal to the multiplication by $j(\chi)$. Moreover (cf. [27, Theorem 3.2]), $i_{P}^{G} \delta_{\chi}$ is irreducible for $\chi$ in an open dense subset of $X(M)$. From (4.3), one deduces that $j$ is not identically zero. Also it is invariant under 
$X(G \mid M)$, by the remark above. Hence, again, its poles and zeros are along a finite number of subtori of $X(M)$ of the form $\chi_{l}^{\prime} X(G \mid M)$ where $\chi_{l}^{\prime} \in X(M)^{G}$. The second part of the lemma follows.

It follows from (4.2) and the proof of the preceding lemma that:

The intertwining integrals and their inverses commute with induced operators from intertwining operators between smooth representations of finite length of $M$.

Let $P^{\prime}=M^{\prime} U^{\prime}$ be a parabolic subgroup of $G$ with $M \subset M^{\prime}, P \subset P^{\prime}$. Let $(\delta, E)$ be a finite length smooth representation of $M$. Let $v$ be an element of $i_{P}^{G} E$. We denote the value at 1 of the element of $i_{P^{\prime}}^{G}\left(i_{M^{\prime} \cap P}^{M^{\prime}} \delta\right)$ associated to $v$ by the induction in stages by $r_{M^{\prime}} v$. Thus for $\chi \in X(M)$, it defines a map denoted again by $r_{M^{\prime}}$ :

$$
r_{M^{\prime}}: i_{P}^{G} E_{\chi} \rightarrow i_{P \cap M^{\prime}}^{M^{\prime}} E_{\chi} .
$$

We will identify $i_{M}^{M} E$ with $E$.

Lemma 4.5. There exists a $(Q, P)$-subset of $X(M), O$, such that for every $\chi \in O$ one has the following property:

For every $G$-submodule, $V$, of $i_{P}^{G} E_{\chi}$ the equality

$$
r_{M}(V)=E
$$

implies the equality

$$
r_{M}\left(A\left(Q, P, \delta_{\chi}\right) V\right)=E .
$$

Proof. Let us take a $(Q, P)$-set as in the previous lemma and let $\chi$ be an element of this $(Q, P)$-set. If $r_{M}\left(A\left(Q, P, \delta_{\chi}\right) V\right)$ is equal to a strict $M$-submodule, $E_{1}$, of $E$, this implies that $A\left(Q, P, \delta_{\chi}\right) V$ is a submodule of $i_{Q}^{G} E_{1}$. By (4.7) one would have $r_{M} V \subset E_{1}$. This leads to a contradiction. This proves that the $(Q, P)$-set of the previous lemma has the required property. The lemma follows.

4.2. Generic Basic Geometric Lemma. Let $P$ (resp., $P^{\prime}$ ) be a parabolic subgroup of $G$ with Levi subgroup $M$ (resp., $M^{\prime}$ ). Let $A$ (resp., $A^{\prime}$ ) be a maximal split torus of $M$ (resp., $M^{\prime}$ ). We choose a maximal compact subgroup $K$ (resp. $K^{\prime}$ ) of $G$ which is the stabilizer of a special point of the apartment associated to $A$ (resp. $\left.A^{\prime}\right)$ in the extended Bruhat-Tits building of $G$. Let us show:

There exists a set of representatives of $P^{\prime} \backslash G / P$ such that for each of its elements, $w$, one has $w \cdot A=A^{\prime}$.

By considering a minimal parabolic subgroup of $G$, contained in $P$ (resp., $P^{\prime}$ ) and containing $A$ (resp., $A^{\prime}$ ), one can reduce to the case where $P$ and $P^{\prime}$ are minimal parabolic subgroups of $G$. Then $P^{\prime}=x . P$ for some element $x$ of $G$. As all maximal split tori in a minimal parabolic subgroup are conjugate by an element of this parabolic subgroup (cf. [5, Theorem 11.6]), one can choose $x$ such that $x . P=P^{\prime}$ and $x . A=A^{\prime}$. On the other hand, by the Bruhat decomposition $G=\bigcup_{w} P w P$ where the $w$ normalize $A$. Hence, $G=\bigcup_{w} P^{\prime} x w P$. Then (4.9) follows from the fact that $x w \cdot A=A^{\prime}$.

We will say that a maximal split torus of $G$ is maximally $\sigma$-split if it contains a maximal $\sigma$-split torus. Such a torus is $\sigma$-stable (cf. [18, Lemma 4.5(ii)]).

We will denote a set of representatives of $P^{\prime} \backslash G / P$ such that for each $w \in W\left(M^{\prime} \backslash G / M\right), w \cdot A=A^{\prime}$ by $W\left(M^{\prime} \backslash G / M\right)$ (although this set is not unique). 
Then $M^{\prime} \cap w \cdot M$ (resp., $M \cap w^{-1} \cdot M^{\prime}$ ) contains $A^{\prime}$ (resp., $A$ ) and is the Levi subgroup of the parabolic subgroup $M^{\prime} \cap w . P$ (resp., $M \cap w^{-1} . P^{\prime}$ ) of $M^{\prime}$ (resp., $M$ ) which contains $A^{\prime}$ (resp., $A$ ).

If $P=M U, P^{\prime}=M^{\prime} U^{\prime}$ are $\sigma$-parabolic subgroups of $G$, one will assume that $A$ (resp., $A^{\prime}$ ) is a maximally $\sigma$-split $\sigma$-stable maximal split torus of $M$ (resp., $M^{\prime}$ ).

Proposition 4.6. Let $P=M U$ and $P^{\prime}=M^{\prime} U^{\prime}$ be parabolic subgroups of $G$ such that $A \subset M, A^{\prime} \subset M^{\prime}$. We denote the set of $A_{M}$-roots in the Lie algebra of $P$ by $\Sigma(P)$. Let $(\delta, E)$ be a smooth representation of $M$ of finite length. Let $w, w^{\prime}$ be two distinct elements of $W\left(M^{\prime} \backslash G / M\right)$. Let $X$ be a complex subtorus of $X(M)$ stable under complex conjugation. We assume that the Lie algebra of $X$ contains at least an element $\nu$ such that $(\nu, \alpha)$ is strictly positive for each element $\alpha$ of the set $\Sigma(P)$. This condition is satisfied in particular if $P=M U$ is a $\sigma$-parabolic subgroup and $X=X(M)_{\sigma}$. Then the following holds:

(i) The set $O_{w, w^{\prime}}$ of elements $\chi$ of $X$ such that:

The $M^{\prime}$-modules $V_{\chi, w}=i_{M^{\prime} \cap w . P}^{M^{\prime}}\left(w j_{M \cap w^{-1} \cdot P^{\prime}} E_{\chi}\right)$ and $V_{\chi, w^{\prime}}=$ $i_{M^{\prime} \cap w^{\prime} . P}^{M^{\prime}}\left(w^{\prime} j_{M \cap w^{\prime-1} P^{\prime}} E_{\chi}\right)$ have disjoint sets of Bernstein parameters (cf. Section 9.2 for the terminology)

is open and dense in $X$. If $(\delta, E)$ is unitary, $O_{w, w^{\prime}} \cap X(M)_{u}$ is dense in $X_{u}:=$ $X \cap X(M)_{u}$.

(ii) If $\chi$ is an element of the open dense subset $O=\bigcap_{w, w^{\prime} \in W\left(M^{\prime} \backslash G / M\right), w \neq w^{\prime}} O_{w, w^{\prime}}$ of $X(M)$, the Jacquet module $j_{P^{\prime}}\left(i_{P}^{G} E_{\chi}\right)$ is isomorphic to the direct sum:

$$
\bigoplus_{w \in W\left(M^{\prime} \backslash G / M\right)} i_{M^{\prime} \cap w . P}^{M^{\prime}}\left(w j_{M \cap w^{-1} \cdot P^{\prime}} E_{\chi}\right) .
$$

Proof. Let $\left\{\Lambda_{1}, \ldots, \Lambda_{p}\right\}$ be the set of Bernstein's parameters of the representation $\left(j_{M \cap w^{-1} \cdot P^{\prime}} \delta, j_{M \cap w^{-1} . P^{\prime}} E\right)$ of $M \cap w^{-1} \cdot M^{\prime}$, where for every $i, \Lambda_{i}=\left(L_{i}, \omega_{i}\right)_{M \cap w^{-1} \cdot M^{\prime}}$, $L_{i}$ is a Levi subgroup of $M \cap w^{-1} \cdot M^{\prime}$ which contains $A$ and $\omega_{i}$ is a cuspidal representation of $L_{i}$, i.e. whose smooth coefficients have a support which is compact modulo the center of $L_{i}$.

We introduce similar data related to $w^{\prime}, L_{j}^{\prime}, \omega_{j}^{\prime}$. Then, using (9.3) and (9.4), one sees:

The set of Bernstein's parameters of the finite length $M^{\prime}$-smooth module $V_{\chi, w}$ is equal to

$$
\left\{\left(w \cdot L_{1}, w\left(\omega_{1} \otimes \chi_{\mid L_{1}}\right)\right)_{M^{\prime}}, \ldots,\left(w \cdot L_{p}, w\left(\omega_{p} \otimes \chi_{\mid L_{p}}\right)\right)_{M^{\prime}}\right\} .
$$

Let us prove that the set $Y=X \backslash O_{w, w^{\prime}}$ is closed in $X$ and has an empty interior. From (4.12), one sees that $\chi \in Y$, if and only if for some $i, j$, one has:

$$
\left(w \cdot L_{i}, w \cdot\left(\omega_{i} \otimes \chi_{\mid L_{i}}\right)\right)_{M^{\prime}} \text { is } M^{\prime} \text {-conjugate to }\left(w^{\prime} \cdot L_{j}^{\prime}, w^{\prime} \cdot\left(\omega_{j} \otimes \chi_{\mid L_{j}^{\prime}}\right)\right)_{M^{\prime}} .
$$

Let $Y_{i, j}$ be the subset of elements of $Y$ satisfying (4.13). Let us assume that $Y_{i, j}$ is nonempty. In particular $w \cdot L_{i}$ is conjugate in $M^{\prime}$ to $w^{\prime} . L_{j}^{\prime}$.

These are two Levi subgroups of $M^{\prime}$ which contain $A^{\prime}$ and which are conjugate under $M^{\prime}$. As two maximal split tori in $w^{\prime} . L_{j}^{\prime}$ are conjugate, these two Levi subgroups of $M^{\prime}$ are conjugate by an element of the normalizer in $M^{\prime}$ of $A^{\prime}$.

By multiplying $w$ by this element of the normalizer in $M^{\prime}$ of $A^{\prime}$, one reduces to the case where these two Levi subgroups of $M^{\prime}, w \cdot L_{i}, w^{\prime} L_{j}^{\prime}$ are equal. Let us 
denote this Levi subgroup of $M^{\prime}$ by $L^{\prime \prime}$. Two cuspidal representations of $L^{\prime \prime}, \omega$, $\omega^{\prime}$ define the same infinitesimal character for $M^{\prime}$ if for some $x$ in the normalizer of $L^{\prime \prime}$ in $M^{\prime}, N_{M^{\prime}}\left(L^{\prime \prime}\right), x \omega$ is equivalent to $\omega^{\prime}$. Hence $\chi \in Y_{i, j}$ if and only if for some $x \in N_{M^{\prime}}\left(L^{\prime \prime}\right)$, that might be chosen to normalize $A^{\prime}$,

$$
x w \omega_{i} \otimes x w \chi_{\mid L^{\prime \prime}} \text { is equivalent to } w^{\prime} \omega_{j}^{\prime} \otimes w^{\prime} \chi_{\mid L^{\prime \prime}} .
$$

For $x$ given, the set $Y_{i, j, x}$ of such $\chi$ is easily seen to be closed because

The characters of these two families of irreducible representations of $L^{\prime \prime}$ vary weakly holomorphically in $\chi$.

As $Y_{i, j, x}$ depends only on the right coset $x L^{\prime \prime}$ and as $N_{M^{\prime}}\left(L^{\prime \prime}\right) / L^{\prime \prime}$ is finite, this implies that $Y_{i, j}$ is closed. Hence $Y$ is closed in $X(M)$ and $O_{w, w^{\prime}}$ is open in $X$.

Let us assume that $O$ is not dense. This implies that $Y$ has a nonempty interior, hence by Baire's Theorem, there exist $w, w^{\prime}, i, j, x$ as above such that $Y_{i, j, x}$ has a nonempty interior.

By multiplying $w^{\prime}$ by $x^{-1}$ one may and one will reduce to the case where $x=1$. From (4.15), one deduces that for all $\chi \in X$, (4.14) holds. In particular it is true for $\chi=1$. Denote by $\omega^{\prime \prime}$ the representation $w \omega_{i}$ of $L^{\prime \prime}$. Then one also concludes that for all $\chi \in X,\left(w^{\prime} \chi_{\mid L^{\prime \prime}}^{-1}\right)\left(w \chi_{\mid L^{\prime \prime}}\right)$ belongs to the finite set of elements $\chi^{\prime \prime}$ of $X\left(L^{\prime \prime}\right)$ such that $\omega^{\prime \prime} \otimes \chi^{\prime \prime}$ is equivalent to $\omega^{\prime \prime}$. Hence, by connectedness,

$$
\text { for all } \chi \in X, w \chi_{\mid L^{\prime \prime}}=w^{\prime} \chi_{\mid L^{\prime \prime}} \text {. }
$$

Let us denote the Lie algebra of $X$ by $\mathfrak{b} \subset\left(\mathfrak{a}_{M}^{\prime}\right)_{\mathbb{C}}$. By differentiation, it implies

$$
w \nu=w^{\prime} \nu, \nu \in \mathfrak{b} .
$$

This might be written:

$$
w^{\prime \prime} \nu=\nu, \nu \in \mathfrak{b}
$$

where $w^{\prime \prime}=w^{\prime-1} w$ is an element of the normalizer of $A$ in $G$. From our hypothesis on $\mathfrak{b}$, one sees that $w^{\prime \prime} \nu=\nu$ for a strictly $P$-dominant element of $\mathfrak{b}, \nu$. But $w^{\prime \prime}$ acts on $\mathfrak{a}_{\mathbb{C}}^{\prime}$ as an element of the Weyl group of $A$, which, by the above, is a product of symmetries with respect to roots orthogonal to $\nu$. The corresponding roots have to be roots of $A$ in the Lie algebra of $M$, by our hypothesis on $\nu$. This implies that $w^{\prime \prime}$ fixes pointwise $\left(\mathfrak{a}_{M}\right)_{\mathbb{C}}^{\prime}$. Hence $w^{\prime \prime}$ is an element of the normalizer of $A$ which fixes pointwise $\mathfrak{a}_{M}$. This implies that it is an element of $M$. As $w=w^{\prime-1} w^{\prime \prime}$, this implies that $w$ and $w^{\prime}$ represent the same element of $P^{\prime} \backslash G / P$. This is a contradiction with our hypothesis. Hence $Y$ has an empty interior and $O$ is dense in $X$. This proves the first statement on $O_{w, w^{\prime}}$. The proof of the statement for $O_{w, w^{\prime}} \cap X_{u}$ is similar.

(ii) By the Basic Geometric Lemma (cf. [25, VI.5.1]) the Jacquet module $j_{P}\left(i_{P}^{G} E_{\chi}\right)$ has a filtration whose associated graded object is the direct sum of the statement. (ii) is an immediate consequence of the definition of $O$ and (9.5).

Lemma 4.7. We keep the notation and the assumptions of the previous lemma.

(i) Let us assume that $(\delta, E)$ is irreducible (resp. irreducible and unitary). There exists an open dense subset of $X$ (resp., $X_{u}$ ) such that for every $\chi$ in this subset, $i_{P}^{G} \delta_{\chi}$ is irreducible.

(ii) Let us assume that $(\delta, E)$ is a finite length smooth representation of $M$. There exists an open dense subset of $X, X^{\prime}$, such that for every $\chi \in X^{\prime}$ and for every $G$-submodule $V$ of $i_{P}^{G} E_{\chi}$ such that $r_{M} V=E_{\chi}$, then $V=i_{P}^{G} E_{\chi}$. 
Proof. (i) follows easily from [27, Theorem 3.2], where no assumption of unitarity on the inducing representation is made, and on our hypothesis on $X$.

(ii) Let $X^{\prime}$ be an open and dense subset of $X$ such that:

1) For every irreducible subquotient of $(\delta, E),(\omega, F)$ and $\chi \in X^{\prime}, i_{P}^{G} \omega_{\chi}$ is irreducible.

2) $X^{\prime}$ is a subset of the set $O$ of the preceding lemma, where we take $P=P^{\prime}$, $M=M^{\prime}$.

The existence of $X^{\prime}$ follows from (i) and from the preceding lemma. We proceed by induction on the length of $E$ to prove that:

An open dense subset, $X^{\prime}$, of $X$ satisfying 1) and 2) above has the properties required by the lemma.

If $E$ is of length one and $\chi \in X^{\prime}, i_{P}^{G} E_{\chi}$ is irreducible. As $V$ is nonzero, one sees that the claim is true in that case.

Now let us assume that (4.17) is true if $E$ is of length $p \geq 1$. Let $E$ be a smooth $M$-module of length $p+1$. Let $\chi$ be an element of $X^{\prime}$. Let $\left(\pi_{1}, V_{1}\right)$ be an irreducible $G$-submodule of $V$. As $\chi \in X^{\prime}, \pi_{1}$ is isomorphic to $i_{P}^{G} \omega_{\chi}$ for some irreducible subquotient $(\omega, F)$ of $(\delta, E)$. This determines a nonzero element, $T$, of $\operatorname{Hom}_{G}\left(i_{P}^{G} F_{\chi}, V\right) \subset \operatorname{Hom}_{G}\left(i_{P}^{G} F_{\chi}, i_{P}^{G} E_{\chi}\right)$. The latter space is isomorphic to $\operatorname{Hom}_{M}\left(j_{P}\left(i_{P}^{G} F_{\chi}\right), E_{\chi}\right)$. But from our hypothesis on $\chi$ and the properties of $X^{\prime}$, $j_{P}\left(i_{P}^{G} F_{\chi}\right)$ splits as a direct sum $\bigoplus_{w \in W(M \backslash G / M)} i_{M \cap w . P}^{M} w j_{M \cap w^{-1} . P} F_{\chi}$. As $\chi$ is an element of $O$, for $w \notin P$, the set of Bernstein parameters of $i_{M \cap w . P}^{M} w j_{M \cap w^{-1} . P} F_{\chi}$ is disjoint from the set of Bernstein parameters of $F_{\chi}$. Hence, one has:

$$
\operatorname{Hom}_{M}\left(j_{P}\left(i_{P}^{G} F_{\chi}\right), E_{\chi}\right) \approx \operatorname{Hom}_{M}\left(F_{\chi}, E_{\chi}\right) .
$$

From this and from the fact that $T$ is nonzero, it follows that $\operatorname{Hom}_{M}\left(F_{\chi}, E_{\chi}\right)$ is nonreduced to zero. This proves that $F$ appears as a submodule of $E$, which we still denote by $F$. Moreover $T$ is the induced map from an element of $\operatorname{Hom}_{M}\left(F_{\chi}, E_{\chi}\right)$ and $V_{1}$ is equal to $i_{P}^{G} F_{\chi}$. Going through the quotient of $V$ by $i_{P}^{G} F_{\chi}$ and applying the induction hypothesis, one gets the result.

We need some notation.

Let $P=M U$ be a parabolic subgroup of $G$, let $(\delta, E)$ be a smooth representation of $M$ and let $x$ be an element of $G$. If there is no ambiguity, we will denote the bijective intertwining operator between $i_{P}^{G} E$ and $i_{x . P}^{G} x E$, which associates to $v \in i_{P}^{G} E$ the element $\lambda(x) v$ of $i_{x . P}^{G} x E$ defined by

$$
\lambda(x) v(g)=v\left(x^{-1} g\right), g \in G,
$$

by $\lambda(x)$. We will also denote, again by abuse of notation, the transpose of the inverse of $\lambda(x)$ by $\lambda(x)$. It intertwines $\left(i_{P}^{G} E\right)^{\prime}$ and $\left(i_{x . P}^{G} x E\right)^{\prime}$.

\section{Proposition 4.8. Generic Basic Geometric Lemma.}

Let $P=M U, P^{\prime}=M^{\prime} U^{\prime}$ be parabolic subgroups of $G$ such that $A \subset M$, $A^{\prime} \subset M^{\prime}$. Let $\Sigma(P)$ be the set of $A_{M}$-roots in the Lie algebra of $P$, let $(\delta, E)$ be a smooth representation of the finite length of $M$ and let $X$ be a complex subtorus of $X(M)$ stable under complex conjugation. We assume that the Lie algebra of $X$, denoted by $\mathfrak{b} \subset\left(\mathfrak{a}_{M}^{\prime}\right)_{\mathbb{C}}$, contains at least an element $\nu$ such that $(\nu, \alpha)$ is strictly positive for each $\alpha$ element of the set $\Sigma(P)$. This in particular is satisfied if $P$ is a $\sigma$-parabolic subgroup and $X=X(M)_{\sigma}$. 
If $w \in W\left(M^{\prime} \backslash G / M\right)$, let us define parabolic subgroups of $G$ by:

$$
P_{w}=\left(M \cap w^{-1} \cdot P^{\prime}\right) U \subset P, P_{w}^{\prime}=\left(M^{\prime} \cap w \cdot P\right) U^{\prime} \subset P^{\prime} .
$$

There exists a dense open subset $O$ of $X$, whose intersection with $X_{u}$ is dense, such that:

(i) For $\chi$ belonging to $O$ and $w$ belonging to $W\left(M^{\prime} \backslash G / M\right)$, the map $\alpha_{\chi, w}$ is well defined from $i_{P}^{G} E_{\chi}$ to $V_{\chi, w}:=i_{M^{\prime} \cap w . P}^{M^{\prime}} w\left(j_{M \cap w^{-1} \cdot P^{\prime}} E_{\chi}\right)$ by:

$$
\alpha_{\chi, w}(v)=r_{M^{\prime}}\left[A\left(P_{w}^{\prime}, w \cdot P_{w}, w j_{M \cap w^{-1} . P^{\prime}} \delta_{\chi}\right)\left(\lambda(w) \circ j_{M \cap w^{-1} \cdot P^{\prime}} \circ v\right)\right],
$$

for $v \in i_{P}^{G} E_{\chi}$. Moreover it goes through the quotient to a surjective morphism of $M^{\prime}$-modules from $j_{P^{\prime}} i_{P}^{G} E_{\chi}$ to $V_{\chi, w}$, that we will denote in the same way. Here we use the normalization of measures as in (3.8) relative to $\left(A^{\prime}, K^{\prime}\right)$.

(ii) For $\chi \in O$, the map

$$
\alpha_{\chi}: j_{P^{\prime}}\left(i_{P}^{G} E_{\chi}\right) \rightarrow \bigoplus_{w \in W\left(M^{\prime} \backslash G / M\right)} V_{\chi, w}
$$

whose components are the $\alpha_{\chi, w}$, is an isomorphism of $M^{\prime}$-modules.

Proof. Let us denote the Levi subgroup of $P_{w}^{\prime}$ which contains $A^{\prime}=w \cdot A$ by $M_{w}^{\prime}$. Then $M_{w}^{\prime}=M^{\prime} \cap w \cdot M$.

From the properties of intertwining integrals (cf. Lemma 4.4), $\alpha_{\chi, w}$ is well defined for a $\chi$ element of $X$ and such that $w \chi_{\mid M_{w}^{\prime}}$ is an element of some $\left(P_{w}^{\prime}, w \cdot P_{w}\right)$ subset $Y_{w}$ of $X\left(M_{w}^{\prime}\right)$.

We denote the set of such $\chi$ by $X_{w}$. Such a set is open in $X$. Let us show that it is dense in $X$. If it was false, the complement of some $\left(P_{w}^{\prime}, w \cdot P_{w}\right)$-subset of $X\left(M_{w}^{\prime}\right)$ would contain the set of $w \chi_{\mid M_{w}^{\prime}}$ when $\chi$ varies in a nonempty open subset of $X$. Thus, by looking to tangent spaces, one would see that $w \mathfrak{b}$ should be contained in the orthogonal subspace of some nonempty collection of roots, $\alpha$, of the maximal split torus of the center of $M_{w}^{\prime}, A_{M_{w}^{\prime}}$, in the Lie algebra of $P_{w}^{\prime}$ and which are not a root in the Lie algebra of $w \cdot P_{w}$. But, by the hypothesis on $X$ in Proposition 4.6, such a root would be trivial on $w \mathfrak{a}_{M}$, as the roots which are orthogonal to $\mathfrak{b}$ are trivial on $\mathfrak{a}_{M}$. Hence it would be a root of $A_{M_{w}^{\prime}}$ in the Lie algebra of the intersection of $P^{\prime}$ with $w \cdot M$. On the other hand, from the definition of $P_{w}$ one sees that:

$$
w \cdot M \cap P^{\prime} \subset w \cdot P_{w} .
$$

Moreover one has:

$$
w \cdot M \cap P^{\prime}=\left(w \cdot M \cap M^{\prime}\right)\left(w \cdot M \cap U^{\prime}\right) .
$$

From the definition of $P_{w}^{\prime}$ one concludes:

$$
w \cdot M \cap P^{\prime} \subset P_{w}^{\prime} .
$$

Hence one sees that $w \cdot M \cap P^{\prime}$ is a subset of $w \cdot P_{w} \cap P_{w}^{\prime}$ and there is no root having the required property. This proves that $X_{w}$ is dense in $X$. Similarly one sees that $X_{w} \cap X_{u}$ is dense in $X_{u}$.

Let us denote the $G$-submodule $\left\{\lambda(w)\left(j_{M \cap w^{-1} \cdot P^{\prime}} \circ v\right) \mid v \in i_{P}^{G} E_{\chi}\right\}$ of $i_{w . P_{w}}^{G}\left(w j_{M \cap w^{-1} P^{\prime}} E_{\chi}\right)$ by $V$. From the surjectivity of $j_{M \cap w^{-1} . P^{\prime}}$ and the surjectivity of $r_{M}$ from $i_{P}^{G} E_{\chi}$ to $E$, one concludes, by "transport de structure", that $r_{M^{\prime} \cap w . M} V=w j_{M \cap w^{-1} P^{\prime}} E_{\chi}$. By Lemma 4.5, one concludes that for $\chi$ belonging to an open dense subset of $X_{w}$ one has:

$$
r_{M^{\prime} \cap w \cdot M}\left(A\left(P_{w}^{\prime}, P_{w}, w j_{M \cap w^{-1} \cdot M^{\prime}} E_{\chi}\right) V\right)=w j_{M \cap w^{-1} \cdot P^{\prime}} E_{\chi} .
$$


As $r_{M^{\prime} \cap w . M}=r_{M^{\prime} \cap w . M} \circ r_{M^{\prime}}$, one concludes that for $\chi$ belonging to this open dense subset of $X_{w}$, the image of $\alpha_{\chi, w}, \alpha_{\chi, w}\left(i_{P}^{G} E_{\chi}\right)$ satisfies:

$$
r_{M^{\prime} \cap w . M} \alpha_{\chi, w}\left(i_{P}^{G} E_{\chi}\right)=w j_{M \cap w^{-1} \cdot P^{\prime}} E_{\chi} .
$$

Then from Lemma 4.7, one deduces that for $\chi$ belonging to an open dense subset of $X_{w}$, the image of $\alpha_{\chi, w}$ is equal to $i_{M^{\prime} \cap w . P}^{M^{\prime}}\left(w j_{M \cap w^{-1} . P^{\prime}} E_{\chi}\right)$. Hence the image of $\alpha$ admits $V_{\chi, w}=i_{M^{\prime} \cap w . P}^{M^{\prime}}\left(w j_{M \cap w^{-1} . P^{\prime}} E_{\chi}\right)$ as a quotient. The fact that $\alpha$ goes through the quotient to $j_{P^{\prime}}\left(i_{P}^{G} E_{\chi}\right)$ follows from the fact that, in the definition of $\alpha_{\chi, w}$, all maps are $G$-morphisms except $r_{M^{\prime}}$, which goes through the quotient to the Jacquet module. This proves (i).

(ii) Using (i) and Proposition 4.6, one sees that for $\chi$ in a dense open subset of $X, O$, whose intersection with $X_{u}$ is dense in $X_{u}, \alpha_{\chi, w}$ is surjective for every $w \in$ $W\left(M^{\prime} \backslash G / M\right)$ and that the various $V_{\chi, w}$ have disjoint sets of Bernstein's parameters. This implies (cf. (9.5)) that the image is equal to the direct sum of $V_{\chi, w}$. So $\alpha$ is a surjective $M^{\prime}$-module map from $j_{P^{\prime}}\left(i_{P}^{G} E_{\chi}\right)$ to $\bigoplus_{w \in W\left(M^{\prime} \backslash G / M\right)} V_{\chi, w}$. On the other hand, by Proposition 4.6. $j_{P^{\prime}}\left(i_{P}^{G} E_{\chi}\right)$ is an $M^{\prime}$-module isomorphic to $\bigoplus_{w \in W\left(M^{\prime} \backslash G / M\right.} V_{\chi, w}$. By looking to the length of modules, one concludes from this that $\alpha$ is bijective.

Let $P$ and $Q$ be two parabolic subgroups of $G$, with a common Levi subgroup $M$. Let $(\delta, E)$ be a smooth representation of the finite length of $M$ such that the operators $A(Q, P, \delta)$ and $A(P, Q, \check{\delta})$ are well defined. Then the restriction of the transposed operator of $A(Q, P, \delta),{ }^{t} A(Q, P, \delta)$ to the space of smooth vectors intertwines $\left(i_{Q}^{G} \delta\right)^{\llcorner}$with $\left(i_{P}^{G} \delta\right)^{\llcorner}$. Using the isomorphism of $i_{P}^{G} \check{E}$ with $\left(i_{P}^{G} E\right)^{\llcorner}$and $i_{Q}^{G} \check{E}$ with $\left(i_{Q}^{G} E\right)^{\curlyvee}(\mathrm{cf}$. (3.10) $)$, the restriction of ${ }^{t} A(Q, P, \delta)$ to the space of smooth vectors defines an intertwining operator between $i_{Q}^{G} \check{E}$ and $i_{P}^{G} \check{E}$ which, by (4.4), is equal to $A(P, Q, \check{\delta})$.

Proposition 4.9. One keeps the notation of the preceding proposition. If $w \in$ $W\left(M^{\prime} \backslash G / M\right)$, let us define two parabolic subgroups of $G$ by

$$
\tilde{P}_{w}=\left(M \cap w^{-1} \cdot P^{\prime-}\right) U \subset P, \tilde{P}_{w}^{\prime}=\left(M^{\prime} \cap w \cdot P\right) U^{\prime-} \subset P^{\prime-} .
$$

There exists a dense open subset $O^{\prime}$ of $X$, whose intersection with $X_{u}$ is dense in $X_{u}$, such that:

(i) For $\chi \in O^{\prime}$ and $w \in W\left(M^{\prime} \backslash G / M\right)$ and $\check{v} \in\left(i_{P}^{G} E_{\chi}\right)^{2}$ :

$$
\beta_{\chi, w}(\check{v}):=\check{r}_{M^{\prime}} \circ{ }^{t} A\left(w \cdot \tilde{P}_{w}, \tilde{P}_{w}^{\prime}, w \cdot j_{M \cap w^{-1} \cdot P^{\prime}} \delta_{\chi}\right) \circ \lambda(w)\left(\check{j}_{M \cap w^{-1} \cdot P^{\prime-}} \circ \check{v}\right)
$$

is a well-defined element of $\left(V_{\chi, w}\right)^{\sim}$ where $V_{\chi, w}:=i_{M^{\prime} \cap w . P}^{M^{\prime}}\left(w j_{M \cap w^{-1} . P^{\prime}} E_{\chi}\right)$. Moreover the map $\beta_{\chi, w}$ goes through the quotient to a surjective morphism of $M^{\prime}$-modules from $j_{P^{\prime}-}\left(\left(i_{P}^{G} E_{\chi}\right)^{\smile}\right)$ to $\left(V_{\chi, w}\right)^{\sim}$ that we will denote in the same way.

(ii) For $\chi \in O^{\prime}$, the map

$$
\beta_{\chi}: j_{P^{\prime}}\left(\left(i_{P}^{G} E_{\chi}\right)^{\swarrow}\right) \rightarrow \bigoplus_{w \in W\left(M^{\prime} \backslash G / M\right)}\left(V_{\chi, w}\right)^{\sim}
$$

whose components are $\beta_{\chi, w}$, is an isomorphism of $M^{\prime}$-modules.

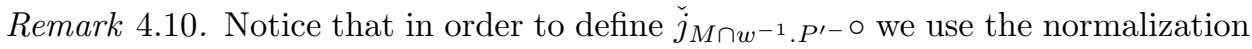
of measures relative to $(A, K)$ (cf. (3.8)). For the intertwining integrals we use $\left(A^{\prime}, K^{\prime}\right)$. When it will be needed, we will make a particular choice of $K^{\prime}$. 
Proof. The fact that $\beta_{\chi, w}(\check{v})$ is a well-defined element of $\left(V_{\chi, w}\right)^{\sim}$ follows from the definitions. The rest of the proof is similar to the proof of the preceding proposition, using the isomorphism of the smooth dual of a parabolically induced representation with the parabolically induced representation of the smooth dual of the inducing representation (cf. (3.10) $)$.

4.3. Generic Basic Geometric Lemma and $H$-forms. We come back to our assumption that the characteristic of $\mathbf{F}$ is different from 2. Let us keep the notation of the preceding proposition. Let $\chi \in O^{\prime}$. We set $V=i_{P}^{G} E_{\chi}$. The Second Adjointness Theorem shows that $\left(i_{P}^{G} E_{\chi}\right)_{P^{\prime}}$ is canonically isomorphic to $\left(\left(\left(i_{P}^{G} E_{\chi}\right)^{\prime}\right)_{P^{\prime-}}\right)^{2}$. From the preceding proposition, the isomorphism $\beta_{\chi}$ determines an isomorphism

$$
\gamma_{\chi}: j_{P^{\prime}}\left(i_{P}^{G} E_{\chi}\right) \rightarrow V_{P^{\prime}, 1}:=\bigoplus_{w \in W\left(M^{\prime} \backslash G / M\right)} V_{\chi, w}
$$

We recall that $\langle., .\rangle_{P^{\prime}}$ is the canonical pairing between $V_{P^{\prime}}$ and $(\check{V})_{P^{\prime}}$. In other words, the isomorphism $\gamma_{\chi}$ is characterized by

$$
\left\langle\check{v}_{P^{\prime-}}, v_{P^{\prime}}\right\rangle_{P^{\prime}}=\left\langle\beta_{\chi}(\check{v}), \gamma_{\chi}(v)\right\rangle, v \in V, \check{v} \in \check{V},
$$

where, in the second member of the equality, the pairing is the natural pairing between $\left(V_{P^{\prime}, 1}\right)^{-}$and $V_{P^{\prime}, 1}$. We denote the composition of $\gamma_{\chi}$ with the projection onto $V_{\chi, w}$ by $\gamma_{\chi, w}$.

Theorem 4.11. We keep the notation of the preceding proposition. We assume moreover that $P$ and $P^{\prime}$ are $\sigma$-parabolic subgroups of $G$. Let $\chi \in O^{\prime}$ and let $\xi$ be an $H$-form on $V:=i_{P}^{G} E_{\chi}$. We define:

$$
\xi_{P^{\prime}-}:=j_{P^{\prime}-} \xi .
$$

We will denote the components of the linear form $\xi^{1}:=\xi_{P^{\prime-}} \circ \gamma_{\chi}^{-1}$ on $V_{P^{\prime}, 1}=$ $\bigoplus_{w \in W\left(M^{\prime} \backslash G / M\right)} V_{\chi, w}$ by $\xi_{P^{\prime-}, w} \in\left(V_{\chi, w}\right)^{\prime}$, where $V_{\chi, w}:=i_{M^{\prime} \cap w . P}^{M^{\prime}}\left(w j_{M \cap w^{-1} \cdot P^{\prime}} E_{\chi}\right)$.

Then one has:

$$
\xi_{P^{\prime-}, w}=\check{r}_{M^{\prime}} \circ^{t} A\left(w \cdot \tilde{P}_{w}, \tilde{P}_{w}^{\prime}, w \cdot j_{M \cap w^{-1} \cdot P^{\prime}} \delta_{\chi}\right) \circ \lambda(w)\left(\check{j}_{M \cap w^{-1} \cdot P^{\prime-}} \circ \xi\right) .
$$

Proof. Let $J$ be a compact open subgroup of $G$ with a $\sigma$-factorization with respect to $\left(P^{\prime}, P^{\prime-}\right)$. Let $v$ be an element of $V^{J}$. Let us compute $\left\langle\xi_{P^{\prime-}}, v_{P^{\prime}}\right\rangle$. First one has, from the definition of $\gamma_{\chi}$ and $\xi^{1}$ :

$$
\left\langle\xi_{P^{\prime-}}, v_{P^{\prime}}\right\rangle=\left\langle\xi^{1}, \gamma_{\chi}\left(v_{P^{\prime}}\right)\right\rangle
$$

From the definition of $\xi_{P^{\prime-}}$ and of the $\sigma$-factorization (cf. (3.7), Proposition 2.3), one has:

$$
\left\langle\xi_{P^{\prime-}}, v_{P^{\prime}}\right\rangle=\left\langle\left(e_{J} \xi\right)_{P^{\prime-}}, v_{P^{\prime}}\right\rangle_{P^{\prime}}
$$

From (4.19), one sees:

$$
\left\langle\xi_{P^{\prime-}}, v_{P^{\prime}}\right\rangle=\left\langle\beta_{\chi}\left(e_{J} \xi\right), \gamma_{\chi}\left(v_{P^{\prime}}\right)\right\rangle .
$$

Let us denote the element of the dual of $V_{P^{\prime}, 1}$ whose components are

$$
\check{r}_{M^{\prime}} \circ^{t} A\left(w \cdot \tilde{P}_{w}, \tilde{P}^{\prime}{ }_{w}, w \cdot j_{M \cap w^{-1} \cdot P^{\prime}} \delta_{\chi}\right) \circ \lambda(w) \circ \check{j}_{M \cap w^{-1} \cdot P^{\prime-} \circ \xi},
$$

by $\xi^{2}$. Using Proposition 3.3 , Proposition 3.2 (i), and the definition of $\beta_{\chi}$ in Proposition 4.9, one easily sees that

$$
\beta_{\chi}\left(e_{J} \xi\right)=e_{J_{M^{\prime}}} \xi^{2}
$$


Hence we get a second expression for $\left\langle\xi_{P^{\prime-}}, v_{P^{\prime}}\right\rangle$ :

$$
\left\langle\xi_{P^{\prime-}}, v_{P^{\prime}}\right\rangle=\left\langle e_{J_{M^{\prime}}} \xi^{2}, \gamma_{\chi}\left(v_{P^{\prime}}\right)\right\rangle, v \in V^{J} .
$$

Together with (4.20), this implies that $\xi^{1}$ and $\xi^{2}$ are equal on $V_{P^{\prime}, 1}^{J_{M^{\prime}}}$. As there are arbitrary small open compact subgroups of $G$ with a $\sigma$-factorization with respect to $\left(P^{\prime}, P^{\prime-}\right)$, this implies that $\xi^{1}=\xi^{2}$. This finishes the proof of the theorem.

\section{TWO KEY LEMMAS AND SOME OF THEIR CONSEQUENCES}

5.1. Families of distributions on $P H$, where $P$ is a parabolic subgroup of $G$. We keep the notation of the preceding subsection. Let $O$ be a nonempty open subset of $X$. A map $\chi \mapsto \xi_{\chi} \in\left(i_{P}^{G} E_{\chi}\right)^{\prime}$ defined on $O$ is said to be weakly holomorphic if for all $v \in i_{K \cap P}^{K} E$, the map $\chi \mapsto\left\langle\xi_{\chi}, v_{\chi}\right\rangle$ is holomorphic on $O$.

We will denote the $\operatorname{map} C_{c}^{\infty}(G) \otimes E \rightarrow i_{P}^{G} E_{\chi}$ denoted by $M_{\delta_{\chi}, P}$ in equation (3.13) by $M_{\chi}$, and we set $\tilde{\xi}_{\chi}:=\xi_{\chi} \circ M_{\chi}$. Let us prove:

Let $f \in C_{c}^{\infty}(G) \otimes E$. Then $\chi \mapsto\left\langle\tilde{\xi}_{\chi}, f\right\rangle$ is holomorphic on $O$; in other words, $\chi \mapsto \tilde{\xi}_{\chi}$ is a weakly holomorphic family of $E$-distributions on $G$.

Let $J$ be a compact open subgroup of $K$ such that $f$ is right and left invariant under $J$. Then $v(\chi):=\left(M_{\chi} f\right)_{\mid K}$ has its values in the finite dimensional space $\left(i_{K \cap P}^{K} E\right)^{J}$. To see that $\chi \rightarrow v(\chi)$ is holomorphic, it is enough to check that for every $k \in K$, $\chi \rightarrow(v(\chi))(k)$ is holomorphic. By using left translates by elements of $K$, one can reduce to $k=1$. But $f \in C_{c}^{\infty}(G) \otimes E$. Hence its restriction to $P$ is invariant under a compact open subgroup $J^{\prime}$ of $P$ and is supported by a finite number of right cosets of $J^{\prime}$ in $P, x_{i} J^{\prime}$. Hence, using the definition of $M_{\chi}$ and the fact that the unramified characters are trivial on compact open subgroups, one sees that:

$$
(v(\chi))(1)=\sum_{i} \chi\left(x_{i}^{-1}\right)\left(\int_{J^{\prime}} \delta_{P}^{1 / 2}\left(x_{i}^{-1}\right) \delta\left(\left(x_{i} p\right)^{-1}\right) f\left(x_{i}\right) d_{r} p\right) .
$$

Hence $\chi \mapsto v(\chi)$ is holomorphic. Then (5.1) follows from the finite dimension of $\left(i_{K \cap P}^{K} E\right)^{J}$ and from our hypothesis on the family $\left(\xi_{\chi}\right)$.

Let $\left(\xi_{\chi}\right)$ be as above and let us assume that every $\xi_{\chi}$ has a zero restriction to the complement of a closed set $F$ of $X$, which is left $P$-invariant. Then (cf. Section 9.1) $\tilde{\xi}_{\chi}$ induces on $F$ an $E$-distribution denoted by $\tilde{\xi}_{F, \chi} \in\left(C_{c}^{\infty}(F) \otimes E\right)^{\prime}$. If $f \in C_{c}^{\infty}(F) \otimes E$, let $f_{1} \in C_{c}^{\infty}(G) \otimes E$ be such that its restriction to $F$ is equal to $f$. Then

$$
\tilde{\xi}_{F, \chi}(f)=\tilde{\xi}_{\chi}\left(f_{1}\right)
$$

From this one concludes that: $\chi \rightarrow \tilde{\xi}_{F, \chi}$ is a weakly holomorphic family of $E$ distributions on $F$. Similarly, if $\Omega$ is a left $P$-invariant open set of $F$, the restriction of $\tilde{\xi}_{F, \chi}$ to $\Omega,\left(\tilde{\xi}_{F, \chi}\right)_{\mid \Omega}$, is a weakly holomorphic family of $E$-distributions on $\Omega$.

Let us make two observations. By [17, Lemma 2.4] one has:

Each parabolic subgroup of $G$ contains a $\sigma$-stable maximal split subgroup of $G$. 
One also has:

Let $A_{0}$ be a maximal split torus of $M$. The morphism of Lie groups from $X(M)$ to $X\left(A_{0}\right)$ given by the restriction has finite kernel. Hence one may view the Lie algebra $\left(\mathfrak{a}_{M}\right)_{\mathbb{C}}^{\prime}$ of $X(M)$ as a subspace of the Lie algebra $\left(\mathfrak{a}_{0}\right)_{\mathbb{C}}^{\prime}$ of $X\left(A_{0}\right)$ (cf. also (2.13) $)$.

The following lemma is one of the two key lemmas in the article.

Lemma 5.1. Let $P$ be a parabolic subgroup of $G$. Let $A_{0}$ be a $\sigma$-stable maximal split torus of $G$ contained in $P$. Let $M$ be the Levi subgroup of $P$ which contains $A_{0}$ and let $U$ be the unipotent radical of $P$.

Let $(\delta, E)$ be a finite length smooth representation of $M$ and let $X$ be a complex subtorus of $X(M)$. Let $O$ be a nonempty open subset of $X$.

Let $\chi \mapsto \xi_{\chi}$ be a weakly holomorphic family, depending on $\chi \in O$, of $E$ distributions on $\mathrm{PH}$. We assume moreover that the family $\left(\xi_{\chi}\right)$ is nonidentically zero and that for every $\chi \in O, \xi_{\chi}$ is $H$-invariant on the right and $\delta_{\chi}$-covariant under P (cf. Section 9.1).

(i) The elements of the Lie algebra $\mathfrak{b}$ of $X$, viewed as a subspace of $\left(\mathfrak{a}_{0}\right)_{\mathbb{C}}^{\prime}$ as in (5.4), are $\sigma$-antiinvariant.

(ii) Moreover if $\mathfrak{b}$ contains a strictly $P$-dominant element, $\nu$ (i.e. such that $(\nu, \alpha)>0$ for every root, $\alpha$, of $A_{M}$ in the Lie algebra of $\left.P\right)$, then $P$ is a $\sigma$-parabolic subgroup of $G$.

Proof. There is no restriction to assume that $O$ is connected and $\xi_{\chi}$ is never equal to zero. Also, by translation by an element of $O$, one can assume that $O$ contains 1. The group $P \times H$ acts on $P H$ by

$$
(p, h) g=p g h^{-1}, g \in P H, p \in P, h \in H .
$$

Hence $P H$ is a homogeneous space under $P \times H$ homeomorphic to $(P \times H) / \operatorname{Diag}(P \cap$ $H)$ by the map $(p, h) \rightarrow p h^{-1}($ cf. [3, Lemma 3.1 (iii)]).

Let us denote the trivial character of $H$ by $\varepsilon_{H}$. Let us define the mean value operation $M_{P \cap H}$ which sends $C_{c}^{\infty}(P \times H) \otimes E$ to $C_{c}^{\infty}(P H) \otimes E$ :

$$
\left(M_{P \cap H} f\right)(p h):=\int_{P \cap H} f\left(p x, x^{-1} h\right) d_{l} x, f \in C_{c}^{\infty}(P \times H) \otimes E,
$$

where $d_{l} x$ is a left invariant Haar measure on $P \cap H$. We define $\xi_{\chi}^{\prime}$ by:

$$
\xi_{\chi}^{\prime}:=\xi_{\chi} \circ M_{P \cap H}
$$

It is a weakly holomorphic family of $E$-distributions on $P \times H$ which are $\left(\delta_{P}^{1 / 2} \otimes\right.$ $\left.\delta_{\chi}\right) \otimes \varepsilon_{H}$-left covariant under $P \times H$.

From Lemma 9.1 there exists $\eta_{\chi} \in E^{\prime}$ such that for all $e \in E$ and for all compact open subgroups, $J$, of $P$ which fixes $e$ under $\delta$, one has

$$
\left\langle\xi_{\chi}^{\prime}, f\right\rangle=\int_{P \times H}\left\langle\eta_{\chi}, \delta(p) f(p, h) e\right\rangle d_{l} p d h=\operatorname{vol}(J)\left\langle\eta_{\chi}, e\right\rangle,
$$

where $f=1_{J} \otimes f_{1} \otimes e \in C_{c}^{\infty}(P \times H) \otimes E$ and $f_{1}$ is a smooth function on $H$ with compact support and with its integral equal to 1 .

As the family $\left(\xi_{\chi}^{\prime}\right)$ is weakly holomorphic, this implies that:

$$
\left(\eta_{\chi}\right) \text { is a weakly holomorphic family of linear forms on } E \text {. }
$$


Also from Lemma 9.1 one deduces:

$$
\left(\delta_{\chi}\right)^{\prime}(p) \eta_{\chi}=\delta_{P \cap H}^{-1}(p) \delta_{P}(p) \eta_{\chi}, p \in P \cap H .
$$

Let $a \in A_{M} \subset A_{0}$ and let $b$ be equal to $a \sigma(a) \in A_{0} \cap H \subset M \cap H$. Then, one has:

$$
\left(\delta_{\chi}\right)^{\prime}(b) \eta_{\chi}=\delta_{P \cap H}^{-1}(b) \delta_{P}(b) \eta_{\chi} .
$$

But $\left(\delta_{\chi}\right)^{\prime}(b)=\chi^{-1}(b) \delta^{\prime}(b)$ so that one has:

$$
\delta^{\prime}(b) \eta_{\chi}=\delta_{P \cap H}^{-1}(b) \delta_{P}(b) \chi(b) \eta_{\chi} .
$$

Let us consider the parabolic subgroup $M \cap \sigma(P)$ of $M$. Its Levi subgroup containing $A_{0}$ is equal to $M \cap \sigma(M)$. Let us prove that:

$$
\eta_{\chi} \in\left(E_{M \cap \sigma(P)}\right)^{\prime}
$$

where $E_{M \cap \sigma(P)}$ is the Jacquet module of $(\delta, E)$ with respect to the parabolic subgroup $M \cap \sigma(P)$ of $M$.

Let $x \in M \cap \sigma(U)$. From [3, Proposition 2.1 (iv)], there exists a unipotent subgroup $V^{\prime}$ of $P \cap \sigma(P), h \in H \cap V^{\prime}$ and $y \in U \cap \sigma(P)$ such that $x=y h$. Then, as $\delta_{\chi}$ is trivial on $U$, one has:

$$
\left(\delta_{\chi}\right)^{\prime}(x) \eta_{\chi}=\left(\delta_{\chi}\right)^{\prime}(y h) \eta_{\chi}=\left(\delta_{\chi}\right)^{\prime}(h) \eta_{\chi}
$$

As $h \in V^{\prime} \cap H$ and $V^{\prime}$ is a unipotent subgroup of $P, h$ is an element of a union of compact subgroups of $P \cap H$. From the fact that a continuous positive character on a topological group is trivial on compact subgroups, one deduces:

$$
\delta_{P \cap H}^{-1}(h) \delta_{P}(h)=1 .
$$

Similarly, as $\chi$ is unramified, one has

$$
\chi(h)=1 .
$$

Hence, from (5.6), one gets

$$
\left(\delta_{\chi}\right)^{\prime}(h) \eta_{\chi}=\eta_{\chi}
$$

As $\chi$ is an unramified character of $M$ and $x$ is an element of the unipotent subgroup of $M, M \cap \sigma(U)$, one has $\chi(x)=1$. From the previous discussion, one sees:

$$
\delta^{\prime}(x) \eta_{\chi}=\eta_{\chi}, x \in M \cap \sigma(U),
$$

so that one has:

$$
\eta_{\chi} \in\left(E_{\delta}^{\prime}\right)^{M \cap \sigma(U)}
$$

which proves (5.8).

The Jacquet module $E_{M \cap \sigma(P)}$ being an $M \cap \sigma(M)$-module of finite length and $b$ being an element of the center of $M \cap \sigma(M)$, the number of generalized eigenvalues of $\delta^{\prime}(b)$ on $\left(E_{M \cap \sigma(U)}\right)^{\prime}$ is finite. From (5.7), one deduces that the map $\chi \mapsto \chi(b)$ is constant on $O$, and equal to 1 as $O$ is connected and contains 1 . In other words we have proved:

$$
\chi(a \sigma(a))=1, \chi \in X, a \in A_{M} .
$$

As $X \subset X(M)$, one views an element $\nu$ of $\mathfrak{b}$ as an element of $\left(\mathfrak{a}_{0}\right)_{\mathbb{C}}^{\prime}$ which vanishes on $\mathfrak{a}_{0}^{M}$. One deduces from (5.9) that:

$$
\nu(X+\sigma(X))=0, X \in \mathfrak{a}_{M}, \nu \in \mathfrak{b} .
$$

Hence the restriction of $\nu$ to $\mathfrak{a}_{M}+\sigma\left(\mathfrak{a}_{M}\right)$ is $\sigma$-antiinvariant. 
Recall that we have choosen a scalar product on $\mathfrak{a}_{0}$ which is invariant under the Weyl group of $\left(G, A_{0}\right)$ and by $\sigma$. Then $\nu \in \mathfrak{b} \subset\left(\mathfrak{a}_{M}^{\prime}\right)_{\mathbb{C}}$ is zero on the orthogonal to $\mathfrak{a}_{M}$, hence also on the orthogonal to $\mathfrak{a}_{M}+\sigma\left(\mathfrak{a}_{M}\right)$. Hence $\nu$ is $\sigma$-antiinvariant. This proves (i).

Now let us assume that $\nu$ is an element of $\mathfrak{b}$ which is strictly $P$-dominant. Then, with the notation of (2.14), one has $P=P_{\nu}$. One sees from the antiinvariance of $\nu$ that $\sigma(P)=P_{-\nu}$, which is clearly opposite to $P$. Hence $P$ is a $\sigma$-parabolic subgroup of $G$.

Lemma 5.2. Let $P=M U$ be a parabolic subgroup of $G$ and let $(\delta, E)$ be a smooth representation of $M$ of finite length Let $X$ be a complex subtorus of $X(M)$ and let $O$ be a nonempty subset of $X$. Let $\chi \mapsto \xi_{\chi}$ be a weakly holomorphic family of $H$-forms on $i_{P}^{G} E_{\chi}$ defined for $\chi \in O$. Let $F$ be the union of the supports of the $\xi_{\chi}$, $\chi \in O$. As these supports are left invariant under $P$ and right invariant under $H$ and as there are only a finite number of $(P, H)$-double cosets, $F$ is closed. We call $F$ the support of the family.

Let $A$ be a maximal split torus in $M$. Let $\Omega$ be a $(P, H)$-double coset of $G$ open in $F$. Then one can choose $x \in \Omega$ such that $A_{x}:=x^{-1}$.A is a $\sigma$-stable maximal split torus contained in $x^{-1}$.P. For such an $x, x^{-1} . M$ is the Levi subgroup of $x^{-1} . P$ which contains $A_{x}$. The conjugation by $x^{-1}$ induces a map $\chi \rightarrow x^{-1} \chi$ from $X$ to the subtorus $x^{-1}$.X of $X\left(x^{-1} . M\right)$.

(i) Then the Lie algebra of $x^{-1}$.X appears as a subspace of $\left(\mathfrak{a}_{x}\right)_{\mathbb{C}}^{\prime}$ made of antiinvariant elements by $\sigma$.

(ii) Moreover if $X$ contains a strictly $P$-dominant element, $x^{-1} . P$ is a $\sigma$-parabolic subgroup.

(iii) With the assumption of (ii), one can choose $x$ such that $A_{x}$ is a maximally $\sigma$-split $\sigma$-invariant maximal split torus in the $\sigma$-stable Levi subgroup of $x^{-1}$.P.

Proof. Let $x$ be an element of $\Omega$. First $x^{-1}$.P contains a $\sigma$-invariant maximal split torus of $G$ (cf. [17, Lemma 2.4]). Two maximal split tori of a parabolic subgroup are conjugate by an element of this subgroup (see [5, Proposition 4.7 and Theorem 4.21]). Hence changing $x$ into $p x$, for a suitable $p \in P$, one can assume that $A_{x}$ is $\sigma$-invariant. The restriction to $\Omega$ of the induced $E$-distribution $\xi_{F, \chi}$ by $\xi_{\chi}$ on $F$ (see Section 9.1) will be denoted by $\zeta_{\chi}$. Then, from the text that follows (5.2), $\lambda\left(x^{-1}\right) \zeta_{\chi}$ (cf. (4.18) for the definition of $\lambda(x)$ ) satisfies the hypothesis of the preceding lemma with $P$ changed in $x^{-1} . P, A_{0}$ in $A_{x}$ and $X$ in $x^{-1} . X$. Then (i) and (ii) are an immediate consequence of the preceding lemma.

Let us prove (iii). We are in the case where $x^{-1} \cdot P$ is a $\sigma$-parabolic subgroup whose $\sigma$-stable Levi subgroup contains a maximally $\sigma$-split $\sigma$-invariant maximal split torus of $G$. This implies (iii).

\subsection{Generic Basic Geometric Lemma for $H$-forms and the role of $\sigma$ - parabolic subgroups.}

Proposition 5.3. Let $P=M U$ (resp., $P^{\prime}=M^{\prime} U^{\prime}$ ) be a $\sigma$-parabolic subgroup and let $A$ (resp., $\left.A^{\prime}\right)$ be a maximally $\sigma$-split maximal split torus of $M$ (resp., $\left.M^{\prime}\right)$. Let $A_{\sigma}$ (resp., $\left.A_{\sigma}^{\prime}\right)$ be the maximal $\sigma$-split torus of $A$ (resp., $\left.A^{\prime}\right)$. We denote the identity component of the set of elements of $X(M)$ which are antiinvariant under $\sigma$ by $X:=X(M)_{\sigma}$. Let $\left(\xi_{\chi}\right)$ be a weakly holomorphic family of $H$-forms on $i_{P}^{G} E_{\chi}$ defined for $\chi$ in an open subset $O$ of $X$. With the notation of Theorem 4.11, let $w$ be an element of $W\left(M^{\prime} \backslash G / M\right)$. Let us assume that $\xi_{P^{\prime-}, w} \neq 0$ where $\xi=\xi_{\chi_{0}}$ for 
some element $\chi_{0}$ of $O \cap O^{\prime}$, where $O^{\prime}$ is as in Theorem 4.11. Then one may change our choice of $w$ in its class in $P^{\prime} \backslash G / P$ in such a way that:

(i) One has $A^{\prime}:=w \cdot A$.

(ii) The group w.P is a $\sigma$-parabolic subgroup of $G$ with a $\sigma$-stable Levi subgroup $w . M$ and $M^{\prime} \cap w . P$ is a $\sigma$-parabolic subgroup of $M^{\prime}$.

(iii) The groups $P_{w}^{\prime}$ and $w . P_{w}$ are $\sigma$-parabolic subgroups of $G$.

(iv) One has the equality $w^{-1} \cdot A_{\sigma}^{\prime}=A_{\sigma}$.

(v) The group $w^{-1} \cdot P^{\prime}$ (resp., $\left.w^{-1} \cdot P^{\prime-}\right)$ is a $\sigma$-parabolic subgroup of $G$ with a $\sigma$ stable Levi subgroup $w^{-1} . M^{\prime}$ and $M \cap w^{-1} . P^{\prime}$ (resp., $M \cap w^{-1} \cdot P^{\prime-}$ ) is a $\sigma$-parabolic subgroup of $M$.

The groups $P_{w}, P_{w}^{\prime}, \tilde{P}_{w}, \tilde{P}_{w}^{\prime}$ are $\sigma$-parabolic subgroups of $G$.

Proof. It will be more convenient for the proof of this proposition to denote $A^{\prime}$ by $A_{1}$ in order to avoid too many 's.

First, as $w \in W\left(M^{\prime} \backslash G / M\right)$, one has $w \cdot A=A_{1}$. Moreover:

The Lie algebra of $X$ is equal to the space $\left(\mathfrak{a}_{M}\right)_{\mathbb{C}}^{\prime-\sigma}$ of $\sigma$-antiinvariant elements of $\left(\mathfrak{a}_{M}\right)_{\mathbb{C}}^{\prime}$ that one can view as a subspace of $\mathfrak{a}_{\mathbb{C}}^{\prime}$ (cf. (2.13) and $(2.18)$ ).

We define $X^{\prime}:=\left\{w \chi_{\mid M^{\prime} \cap w . M} \mid \chi \in X\right\}$ which is closed in $X\left(M^{\prime} \cap w \cdot M\right)$ (cf. (2.11) ). By looking to a differential, one sees that on an open neighborhood of $\chi_{0}$ in $O \cap O^{\prime}$, the map $\chi \mapsto w \chi_{\mid M^{\prime} \cap w . M}$, from $X$ to $X^{\prime}$, is an isomorphism whose inverse, defined by $O^{\prime \prime} \subset X^{\prime}$, will be denoted $\chi^{\prime} \mapsto w^{-1} \chi^{\prime}$. Let us show that the family $\chi^{\prime} \rightarrow\left(\xi_{w^{-1} \chi^{\prime}}\right)_{P^{\prime-}, w}$ defined by $O^{\prime \prime}$ is weakly holomorphic. It is enough to prove that for all $e$ elements of the compact realization of $i_{M^{\prime} \cap w . P}^{M^{\prime}} w j_{M \cap w^{-1} . P^{\prime}} E_{\chi}$, the map $\chi \mapsto\left\langle\left(\xi_{\chi}\right)_{P^{\prime-}, w}, e_{w \chi}\right\rangle$ is holomorphic on $O$. We choose a compact open subgroup of $G, J$, with a $\sigma$-factorization for $\left(P^{\prime}, P^{\prime-}\right)$ such that $e$ is fixed by $J_{M^{\prime}}$. One starts by using (3.7) and then uses the fact that $\left(e_{J} \xi_{\chi}\right)$ is a holomorphic family of $J$-fixed vectors. One deduces from Proposition 4.9 that $\left\langle\left(\xi_{\chi}\right)_{P^{\prime-}, w}, e_{w \chi}\right\rangle$ is weakly holomorphic if for all $v$ in the compact realization of $i_{P}^{G} \check{E}$, the map $\chi \mapsto\left\langle\beta_{\chi, w}(\check{v}), e_{w \chi}\right\rangle$ is holomorphic on $O^{\prime}$, where $\beta_{\chi, w}(\check{v})=\left(\check{v}_{\chi}\right)_{P^{\prime-}, w}$. This follows easily from the definition of $\beta_{\chi, w}(\check{v})$ (cf. Proposition 4.9) and from the holomorphy properties of the intertwining integrals.

As $P$ is a $\sigma$-parabolic subgroup, $X$ contains strictly $P$-dominant elements and $X^{\prime}$ contains a strictly $M^{\prime} \cap w . P$-dominant element. The conclusion of the preceding lemma, applied to $M^{\prime}$ instead of $G$ and $X^{\prime}$ instead of $X$, asserts that there exists $m^{\prime} \in M^{\prime}$ such that $A_{2}:=m^{\prime} w \cdot A=m^{\prime} . A_{1}$ is a maximally $\sigma$-split, $\sigma$-stable, maximal split torus of $M^{\prime}, Q:=m^{\prime} .\left(M^{\prime} \cap w . P\right)$ is a $\sigma$-parabolic subgroup of $M^{\prime}, m^{\prime} . X^{\prime}$ and hence $m^{\prime} w\left(\mathfrak{a}_{M}\right)^{\prime-\sigma} \subset\left(\mathfrak{a}_{2}\right)^{\prime}$ is made of $\sigma$-antiinvariant elements, where $\left(\mathfrak{a}_{M}\right)^{\prime-\sigma}$ is the space of antiinvariant elements of $\left(\mathfrak{a}_{M}\right)^{\prime}$. Then the half sum of the roots of $A_{2}$ in the Lie algebra of $Q, \rho_{Q}$, satisfies:

$$
\rho_{Q} \in\left(\mathfrak{a}_{2}\right)^{\prime-\sigma} .
$$

Two maximally $\sigma$-split $\sigma$-stable maximal split tori of $M^{\prime}$ are conjugate by an element of $M^{\prime}$ which conjugates their maximal $\sigma$-split tori, because two maximal $\sigma$-split tori are conjugate, and two maximal split tori in the centralizer of a maximal $\sigma$-split torus are conjugate. So one can choose $m^{\prime \prime}$ in $M^{\prime}$ such that $m^{\prime \prime} . A_{2}=A^{\prime}$ and $m^{\prime \prime}\left(\mathfrak{a}_{2}\right)^{\prime-\sigma}=\left(\mathfrak{a}_{1}\right)^{\prime-\sigma}$. Then from (5.12), one sees that the parabolic subgroup of $M, Q^{\prime}:=\left(m^{\prime \prime} m^{\prime} w \cdot M\right) \cap P^{\prime}$ contains $A_{1}$ and the half sum of the roots of $A_{1}$ in 
its Lie algebra $\rho_{Q^{\prime}}$ is an element of $\left(\mathfrak{a}_{1}\right)^{\prime-\sigma}$. Using (2.14), one sees that it is a $\sigma$-parabolic subgroup of $M$. One will change $w$ to $m^{\prime \prime} m^{\prime} w$. Hence one has:

$$
w \cdot A=A_{1}, w\left(\mathfrak{a}_{M}\right)^{\prime-\sigma} \subset\left(\mathfrak{a}_{1}\right)^{\prime-\sigma} .
$$

Let us show that $w$ satisfies (ii) and (iii). Let $\rho_{P} \in \mathfrak{a}_{M}^{\prime} \subset \mathfrak{a}^{\prime}$ be the half sum of roots of $A$ in the Lie algebra of $P$. Similarly we define $\rho_{w . P_{w}}, \rho_{P_{w}^{\prime}}$ and $\rho_{P^{\prime} \cap w . M}$ with respect to $A_{1}$. These are elements of $\left(\mathfrak{a}_{1}\right)^{\prime}$. One has

$$
\rho_{w \cdot P_{w}}=\rho_{P^{\prime} \cap w \cdot M}+w \rho_{P} .
$$

As $P$ is a $\sigma$-parabolic subgroup of $G$, one has $\rho_{P} \in\left(\mathfrak{a}_{M}\right)^{\prime-\sigma}$. From (5.13), one deduces that:

$$
w \rho_{P} \in\left(\mathfrak{a}_{1}\right)^{\prime-\sigma} \text { is } \sigma \text {-antiinvariant. }
$$

It follows that $w \cdot P$ is a $\sigma$-parabolic subgroup of $G$. The group $w \cdot M$ is the Levi subgroup of $w \cdot P$ which contains $A_{1}=w \cdot A$, whose Lie algebra is the sum of the $A_{1}$-weight spaces for weights $\alpha$ which are equal to zero or to an $A_{1}$-root $\alpha$ such that $\left(w \rho_{P}, \alpha\right)=0$. As $w \rho_{P}$ is $\sigma$-antiinvariant, one sees that $w \cdot M$ is $\sigma$-invariant. As $P^{\prime}$ is a $\sigma$-parabolic subgroup of $G, \sigma\left(P^{\prime}\right) \cap P^{\prime}=M^{\prime}$, which implies:

$$
\sigma\left(P^{\prime} \cap w \cdot M\right) \cap P^{\prime} \cap w \cdot M=M^{\prime} \cap w \cdot M .
$$

As $M^{\prime} \cap w \cdot M$ is the Levi subgroup of the parabolic subgroup $P^{\prime} \cap w \cdot M$ of $w \cdot M$ which contains $A^{\prime}$, this implies that $P^{\prime} \cap w \cdot M$ is a $\sigma$-parabolic subgroup of $w \cdot M$ with the $\sigma$-stable Levi subgroup equal to $w \cdot M \cap M^{\prime}$. Hence

$$
\rho_{P^{\prime} \cap w . M} \text { is } \sigma \text {-antiinvariant. }
$$

From (5.14), (5.15), and (5.16), one sees that $\rho_{w \cdot P_{w}} \in\left(\mathfrak{a}_{1}\right)^{\prime}$ is $\sigma$-antiinvariant. It follows from (2.14) that $w \cdot P_{w}$ is a $\sigma$-parabolic subgroup of $G$. One easily sees that its $\sigma$-stable Levi subgroup is $M^{\prime} \cap w . M$.

Similarly one proves that $P_{w}^{\prime}$ is a $\sigma$-parabolic subgroup of $G$, by using the equality:

$$
\rho_{P_{w}^{\prime}}=\rho_{M^{\prime} \cap w . P}+\rho_{P^{\prime}}
$$

and that $P^{\prime}$ (resp., $M^{\prime} \cap w . P$ ) is a $\sigma$-parabolic subgroup of $G$ (resp., $M^{\prime}$ ), as w.P is a $\sigma$-parabolic subgroup of $G$ from (5.15) and $w \cdot M$ is its $\sigma$-stable Levi subgroup.

Altogether we have found a choice of $w$ which satisfies (i), (ii) and (iii). We will modify our preceding choice of $w$ to get one which will also satisfy (iv).

Let $w$ be as above. Then $(w \cdot P) w H=w P H$ is open in $G$. Moreover $w \cdot P$ is a $\sigma$-parabolic subgroup of $G$, and $\left(A_{1}\right)_{\sigma}$ is a maximal $\sigma$-split torus contained in $w . P$, hence contained in its $\sigma$-stable Levi subgroup $w . M$. As any $(w \cdot P, H)$-open orbit has a representative which is $\left(A_{1}\right)_{\sigma}$-good (cf. (2.22) $)$, there exists $p^{\prime}=w . p$ with $p \in P$ such that $w^{\prime}:=(w \cdot p) w=w p$ satisfies the fact that $w^{\prime-1} \cdot\left(A_{1}\right)_{\sigma}$ is $\sigma$ split. But $w^{-1} .\left(A_{1}\right)_{\sigma} \subset p^{-1} . A \subset P$. As $w^{\prime-1} \cdot\left(A_{1}\right)_{\sigma}$ is $\sigma$-split, it is $\sigma$-stable hence contained in $M=P \cap \sigma(P)$. Hence $w^{\prime-1} \cdot\left(A_{1}\right)_{\sigma}$ is a maximal $\sigma$-split torus in $M$. Then, as all maximal $\sigma$-split tori in $M$ are conjugate (cf. [17, Proposition 1.16]), we can choose an element $m$ of $M$, such that $w^{\prime \prime}=w^{\prime} m$ satisfies $w^{\prime \prime-1} \cdot\left(A_{1}\right)_{\sigma}=A_{\sigma}$. Then $w^{\prime \prime-1} . A^{\prime}$ is contained in the centralizer of $A_{\sigma}$ which is contained in $M$. Hence one can choose $m_{1}$ elements of this centralizer such that $w_{1}:=w^{\prime \prime} m_{1}$ is such that $w_{1}^{-1} \cdot A_{1}=A$ and $w_{1}^{-1} \cdot\left(A_{1}\right)_{\sigma}=A_{\sigma}$. Hence, as $\rho_{P} \in \mathfrak{a}^{\prime-\sigma}, w_{1} \rho_{P}$ is $\sigma$-antiinvariant and $w_{1} . P$ is a $\sigma$-parabolic subgroup of $G$. As above, one also sees that it implies that $w_{1} . M$ is its $\sigma$-stable Levi subgroup. Then, as above, one sees that $w_{1}$ satisfies 
(ii) and (iii). As $P^{\prime}$ is a $\sigma$-parabolic subgroup of $G, \rho_{P^{\prime}}$ is an element of $\left(\mathfrak{a}_{1}\right)^{\prime-\sigma}$ and one gets $w_{1}^{-1} \rho_{P^{\prime}} \in \mathfrak{a}^{\prime-\sigma}$. One sees, as above, that $w_{1}^{-1} \cdot P^{\prime}$ is a $\sigma$-parabolic subgroup of $G$ with a $\sigma$-stable Levi subgroup equal to $w_{1}^{-1} \cdot M^{\prime}$. This easily implies that $M \cap w_{1}^{-1} \cdot P^{\prime}$ is a $\sigma$-parabolic subgroup of $M$ with a $\sigma$-stable Levi subgroup equal to $M \cap w_{1}^{-1} \cdot P^{\prime}$.

Then the assertions on $P_{w_{1}}, P_{w_{1}}^{\prime}, \tilde{P}_{w_{1}}, \tilde{P}_{w_{1}}^{\prime}$ follow easily. Thus $w_{1}$ has the required properties.

5.3. Intertwining integrals and support of families of $H$-forms. An ordered pair $\left(P=M U, P^{\prime}=M U^{\prime}\right)$ of parabolic (resp., $\sigma$-parabolic) subgroups of $G$, is said to be adjacent (resp., $\sigma$-adjacent) if there is a unique reduced $A_{M}$-root (resp., a reduced $\left(A_{M}\right)_{\sigma}$-root), $\alpha$, which is positive for $P$ and negative for $P^{\prime}$. We denote by $A^{\alpha}$ the group of $\mathbf{F}$-points of the identity component of the kernel of $\alpha$ in $\underline{A}_{M}$ (resp., $\left.\left(\underline{A}_{M}\right)_{\sigma}\right)$ and by $M^{\alpha}$ the centralizer of $A^{\alpha}$ in $G$. The group $P^{\alpha}$ generated by $P$ and $P^{\prime}$ is a parabolic (resp. $\sigma$-parabolic) subgroup of $G$ with $M^{\alpha}$ as a Levi subgroup (resp. the $\sigma$-stable Levi subgroup) and its unipotent radical $U^{\alpha}$ is contained in $U \cap U^{\prime}$. It is easy to see that $\alpha$ is $P$-simple.

A minimal string of parabolic (resp., $\sigma$-parabolic) subgroups of $G$ between two parabolic (resp., $\sigma$-parabolic) subgroups of $G, P=M U, P^{\prime}=M U^{\prime}$, is a sequence $\left(P_{i}\right)_{i=0, \ldots, r}$ of parabolic (resp., $\sigma$-parabolic) subgroups of $G$, such that $P_{0}=P, P_{r}=$ $P^{\prime}$ and $\left(P_{i}, P_{i+1}\right)$ is adjacent (resp., $\sigma$-adjacent) for $i=0, \ldots, r-1$. Such a string always exists (cf. [22, before Theorem 4.2 for parabolic subgroups; it works in a similar manner for $\sigma$-parabolic subgroups).

The next lemma is the second key lemma mentioned in the Introduction. It was suggested by a geometric result of Matsuki (cf. [24, Lemma 3]).

Lemma 5.4. Let $P=M U$ and $Q=L V$ be two $\sigma$-parabolic subgroups of $G$, with $P \subset Q$. Let $(\delta, E)$ be a smooth irreducible representation of $M$. Let $P^{\prime}=M U^{\prime}$ be another $\sigma$-parabolic subgroup of $G$ such that $\left(P, P^{\prime}\right)$ is $\sigma$-adjacent, and let $\alpha$ be the unique reduced $\left(A_{M}\right)_{\sigma}$-root which is positive for $P$ and negative for $P^{\prime}$. One assumes that the restriction $\alpha_{\mid a_{L}^{-\sigma}}$ of $\alpha$, to $\mathfrak{a}_{L}^{-\sigma}$ is nonzero, where $\mathfrak{a}_{L}^{-\sigma}$ is the subspace of elements of $\mathfrak{a}_{L}$ antiinvariant under $\sigma$.

We denote the identity component of the set of $\sigma$-antiinvariant elements of $X(L)$ by $X(L)_{\sigma}$.

Let $\chi \mapsto \xi_{\chi}$ be a weakly holomorphic family of $H$-forms on $i_{P}^{G} E_{\chi}$ defined for $\chi$ in an open subset, $O$, of $X(L \mid M)_{\sigma}:=\left\{\chi_{\mid M} \mid \chi \in X(L)_{\sigma}\right\}$. Let us assume that the support of every $\xi_{\chi}$ has an empty interior in $G$.

Then one has the following:

(i) The set $O^{\prime}$ of $\chi \in O$ such that $A\left(P, P^{\prime}, \delta_{\chi}\right)$ has no pole is an open and dense subset of $O$.

(ii) If $\chi \in O^{\prime}$, the support of $\xi_{\chi} \circ A\left(P, P^{\prime}, \delta_{\chi}\right)$, which is an $H$-form on $i_{P^{\prime}}^{G} E_{\chi}$, has an empty interior in $G$.

(iii) Let $Q^{\prime}$ be the $\sigma$-adjacent $\sigma$-parabolic subgroup of $G$ determined by the $Q$ simple $\left(A_{L}\right)_{\sigma}$-root $\alpha_{\mid a_{L}^{-\sigma}}$. Then $P^{\prime} \subset Q^{\prime}$ and $\left(Q, Q^{\prime}\right)$ are $\sigma$-adjacent.

Proof. The fact that $O^{\prime}$ is dense follows from the fact that $\alpha$ restricted to $\mathfrak{a}_{L}^{-\sigma}$ is nonzero and from Lemma 4.4. This proves (i).

Let us show that the union of the $(P, H)$-double cosets $P x_{i} H$ which are open in the support $F$ of the family $\xi_{\chi}, \chi \in O^{\prime}$ is dense in this support. In fact if it 
was false, the complementary of the union of the $P x_{i} H$ would contain a $(P, H)$ invariant subset open in $F$. Then it contains an open $(P, H)$-double coset in $F$ (see e.g. [3, Lemma 3.1 (ii)]). This is impossible due to our definition of $P x_{i} H$. This proves our claim.

From Lemma 4.2, the support of the family $\left(\xi_{\chi} \circ A\left(P, P^{\prime}, \delta, \chi\right)\right)_{\chi \in O^{\prime}}$ is contained in $c l\left(P^{\alpha} F\right)$, hence in the union of $c l\left(P^{\alpha} x_{i} H\right)$ as for $A, B$, subsets of $G, \operatorname{cl}(A c l(B))=$ $\operatorname{cl}(A B)$ and $\operatorname{cl}(A \cup B)=\operatorname{cl}(A) \cup \operatorname{cl}(B)$. Hence it suffices to show that:

For all $x=x_{i}$ one has $P^{\alpha} x H$ with empty interior.

Let $A$ be a maximally $\sigma$-split torus $\sigma$-stable maximal split torus in $M$, which is automatically a maximally $\sigma$-split $\sigma$-stable maximal split torus in $G$ as $P$ is a $\sigma$ parabolic subgroup. As any parabolic subgroup contains a $\sigma$-invariant maximal split torus, we may and we will choose $x$ in its double $(P, H)$-coset such that $x^{-1}$.A is $\sigma$-stable. The Lie algebra of $X:=X(L \mid M)_{\sigma}$ is identified with $\left(\mathfrak{a}_{L}^{\prime}\right)_{\mathbb{C}}^{-\sigma}$. We can apply Lemma 5.2 (ii) to the family $\left(\xi_{\chi}\right)_{\chi \in O^{\prime}}$ and one sees, using (2.18), that:

$$
x^{-1}\left(\mathfrak{a}_{L}^{-\sigma}\right) \subset\left(x^{-1} \mathfrak{a}\right)^{-\sigma} .
$$

One sets: $P_{x}=x^{-1} \cdot P, P_{x}^{\prime}=x^{-1} \cdot P^{\prime}$, etc.. Here we denote the Lie algebra of the algebraic group $\underline{G}$ such that $G=\underline{G}(\mathbf{F})$ by $g$. By abuse of terminology we will say that $g$ is the Lie algebra of $G$ (notice that this notion is different from the one used in Section 10). We use a similar terminology for the subgroups of $G$ which are the groups of F-points of a subgroup of $\underline{G}$ defined over $\mathbf{F}$.

Let $g(\alpha)$ (resp., $g(-\alpha)$ ) be the Lie algebra of $U \cap M^{\alpha}$ (resp., $\left.U^{\prime} \cap M^{\alpha}\right)$. Similarly let $\underline{g}\left(x^{-1} \alpha\right)$ (resp., $\left.\underline{g}\left(-x^{-1} \alpha\right)\right)$ be the Lie algebra of $U_{x} \cap M_{x}^{\alpha}$ (resp., $\left.U_{x}^{\prime} \cap M_{x}^{\alpha}\right)$. One has:

$$
\underline{p}_{x}^{\alpha}=\underline{g}\left(-x^{-1} \alpha\right)+\underline{p}_{x} .
$$

We fix $Y_{x}=x^{-1} Y \in x^{-1}\left(\mathfrak{a}_{L}^{-\sigma}\right)$, where $Y$ is a strictly $Q$-dominant element in $\left(\mathfrak{a}_{L}^{\prime}\right)^{-\sigma}$ which is canonically identified to $\mathfrak{a}_{L}^{-\sigma}$ (cf. (2.18)). Then an $A_{x}$-root $\beta$ is such that $\beta\left(Y_{x}\right)>0$ if and only if the corresponding root space is contained in $\underline{v}_{x}$. But $\underline{g}(\alpha) \subset \underline{v}$, as $P \subset Q$ and $\alpha_{\mid \mathfrak{a}_{L}} \neq 0$. So one has $\left(x^{-1} \alpha\right)\left(Y_{x}\right)>0$. Let us prove that:

$$
\underline{h}+\underline{p}_{x}=\underline{h}+\underline{p}_{x}^{\alpha} \text {. }
$$

The only thing to prove is that $\underline{g}\left(-x^{-1} \alpha\right) \subset \underline{h}+\underline{p}_{x}$. Let $\beta$ be a root of $A_{x}$ in $\underline{g}\left(-x^{-1} \alpha\right)$, so that $\beta\left(Y_{x}\right)<0$ by what has been said above. Let $Z$ be an element of the corresponding weight space in $g$. Let $\sigma \beta$ be the $A_{x} \operatorname{root} \beta \circ \sigma$. Then $\sigma \beta\left(Y_{x}\right)=\beta\left(\sigma\left(Y_{x}\right)\right)$. But as $x^{-1}\left(\mathfrak{a}_{L}^{-\sigma}\right)$ is contained in the space of $\sigma$-antiinvariant elements (cf. (5.18) ), one has $\sigma\left(Y_{x}\right)=-Y_{x}$, and $\sigma \beta\left(Y_{x}\right)=-\beta\left(Y_{x}\right)$ is strictly positive. Hence $\sigma(Z)$ is an element of $\underline{v}_{x} \subset \underline{p}_{x}$. Consequently one has:

$$
Z=(Z+\sigma(Z))-\sigma(Z) \in \underline{h}+\underline{p}_{x},
$$

which proves (5.19).

Let us assume that $P_{x}^{\alpha} H$ has a nonempty interior. We will prove that it leads to a contradiction. From (2.22) and Lemma 2.2 (iv), one deduces that $P_{x}^{\alpha}$ is a $\sigma$-parabolic subgroup of $G$. Hence (9.6) implies that $\underline{P}_{x}^{\alpha}$ is a $\sigma$-parabolic of $\underline{G}$ and (9.7) implies

$$
\underline{h}+\underline{p_{x}^{\alpha}}=\underline{g} .
$$

Then, together with (5.19), it implies that $\underline{h}+\underline{p_{x}}=\underline{g}$. Hence (cf. Lemma 9.3), $\underline{H} \underline{P}_{x}$ is open. From Lemma 9.4 one sees that $\overline{P x H}$ would be open in $G$. This is 
a contradiction with our hypothesis on the support of $\xi$, which implies (5.17). We have thus proved (ii).

The assertion on $Q^{\prime}$ in (iii) being clear, this proves the lemma.

Lemma 5.5. We keep the notation of the previous lemma, except that $P^{\prime}$ is not necessarily adjacent to $P$. We assume that every $\left(A_{M}\right)_{\sigma}$-root which is positive for $P^{\prime}$ and negative for $P$ satisfies $\alpha_{\mid \mathfrak{a}_{L}^{-\sigma}} \neq 0$. Then the same conclusion as in (i) and (ii) of the previous lemma is valid.

Proof. Let $P_{0}, \ldots, P_{r}$ be a minimal string of $\sigma$-parabolic subgroups between $P$ and $P^{\prime}$. We will prove by induction on $i$ that for $\chi \in O^{\prime}$, the support of $A\left(P_{i}, P, \delta_{\chi}\right) \xi_{\chi}$ has an empty interior in $G$ and we will define $\sigma$-parabolic subgroups of $G, Q_{0}=$ $Q, \ldots, Q_{r}$, with a $\sigma$-stable Levi subgroup $L$, such that:

The family $\left(\xi_{\chi}^{i}\right):=\left(\xi_{\chi} \circ A\left(P_{i}, P, \delta_{\chi}\right)\right)$ is such that $\left(P_{i}, P_{i+1}\right), Q_{i}$ satisfy the hypothesis of the previous lemma, by the induction hypothesis. The lemma follows by using this previous lemma.

\section{FAMILIES OF $H$-FORMS ON REPRESENTATIONS INDUCED FROM $\sigma$-PARABOLIC SUBGROUPS AND $B$-MATRICES}

6.1. Families of $H$-forms on representations induced from $\sigma$-parabolic subgroups. Let $P=M U$ be a $\sigma$-parabolic subgroup of $G$. Let $(\delta, E)$ be a smooth representation of $M$ with finite length. Let $\mathcal{W}_{M}^{G}$ be a set of representatives of the open $(P, H)$-double cosets as in (2.22): here $A_{\sigma}$ is a maximal $\sigma$-split torus of $M$. As remarked in Lemma 2.2 if $x \in \mathcal{W}_{M}^{G}, x^{-1} . P$ is a $\sigma$-parabolic subgroup of $G$. We define

$$
E^{\prime}(\delta, H):=\bigoplus_{x \in \mathcal{W}_{M}^{G}} E^{M \cap x . H} .
$$

We have the following mild generalization of [3. Theorem 2.8], that one gets in an entirely similar way (see the comments following the statement). Notice that this statement is true assuming only that the characteristic of $\mathbf{F}$ is different from 2, as is shown by the examination of the proof.

Let us give a definition.

If $B$ is a split torus we denote the real vector space denoted $\mathfrak{a}_{B}$ in (2.3) by $\mathfrak{b}$.

The lattice $\Lambda(B)$ (cf. (2.2) for its definition) is identified with a subset of $\mathfrak{b}$ (cf. (45)), and if $B$ is a subtorus of the split torus $A$ one has $\mathfrak{b} \subset \mathfrak{a}$.

We will use the identification of the Lie algebra of $X(M)_{\sigma}$ and $\left(\mathfrak{a}_{M}^{-\sigma}\right)_{\mathbb{C}}$ given by a suitable scalar product on $\mathfrak{a}_{M}$ (see (2.13) and (2.18) ).

Proposition 6.1. Let us assume that we are given a $\sigma$-split torus $B \subset A_{M}$, and a complex subtorus $X$ of $X(M)_{\sigma}$. We assume that the Lie algebra of $X$ is identified with $\mathfrak{b}_{\mathbb{C}} \subset\left(\mathfrak{a}_{M}^{-\sigma}\right)_{\mathbb{C}}$ and that the lattice $\Lambda(B)$ contains strictly $P$-dominant elements.

(i) Let $\chi \in X$. Let us denote the subspace of elements of $i_{P}^{G} E_{\chi}$ whose support is contained in the union of the open $(P, H)$-double cosets by $J_{\chi}$.

There is a canonical linear isomorphism between $E^{\prime}(\delta, H)$ and $J_{\chi}^{\prime H}$ which associates to $\eta \in E^{\prime M \cap x . H}$ the element $\xi^{\prime}\left(P, \delta_{\chi}, \eta\right)$ of $J_{\chi}^{\prime H}$ defined by:

$$
\xi^{\prime}\left(P, \delta_{\chi}, \eta\right)(\varphi)=\int_{\left(H \cap x^{-1} . M\right) \backslash H}\langle\varphi(x h), \eta\rangle d h, \varphi \in J_{\chi} .
$$


(ii) There exists an open dense subset, $O_{0}$, of $X$ such that for $\chi \in O_{0}, \xi^{\prime}\left(P, \delta_{\chi}, \eta\right)$ extends uniquely to an $H$-form, $\xi\left(P, \delta_{\chi}, \eta\right)$, on $i_{P}^{G} E_{\chi}$. In particular for $\chi \in O_{0}$, every $H$-form on $i_{P}^{G} E_{\chi}$ whose restriction to $J_{\chi}$ is equal to zero vanishes.

(iii) Moreover there exists a polynomial function, $b$, on $X$, such that for every $v \in i_{K \cap P}^{K} E$ and $\eta \in E^{\prime}(\delta, H), \chi \mapsto b(\chi)\left\langle\xi\left(P, \delta_{\chi}, \eta\right), v_{\chi}\right\rangle$ extends to a polynomial function on $X$.

The main point for this generalization of [3, Theorem 2.8] is the generalization of [3, Lemma 2.5] with our $X$. But it is straightforward by changing $A$ to $B$ in its proof and the projection $p_{\mathfrak{a}}$ to the orthogonal projection $p_{\mathfrak{b}}$ on $\mathfrak{b}$.

Then, we will see that one has, for a suitable normalization of the measures in Proposition 6.1

$$
\begin{aligned}
& \text { For all } \eta=\left(\eta_{x}\right)_{x \in \mathcal{W}_{M}^{G}} \text { and for all } x \in \mathcal{W}_{M}^{G}, \check{r}_{M}\left(\left(i_{P}^{G} \delta_{\chi}\right)^{\prime}(x) \xi\left(P, \delta_{\chi}, \eta\right)\right)= \\
& \eta_{x} \text {. }
\end{aligned}
$$

For $x=1$, it follows from Section 9.1 and [3, Equation (2.33)]. Now the assertion reduces to the one for $x=1$ applied to the $x . H$-fixed linear form $\left(i_{P}^{G} \delta_{\chi}\right)^{\prime}(x) \xi\left(P, \delta_{\chi}, \eta\right)$.

\subsection{B-matrices.}

Proposition 6.2. We keep the notation of the preceding proposition. Let $Q$ be a $\sigma$-parabolic subgroup of $G$ with a $\sigma$-stable Levi subgroup equal to $M$. There exists a rational function $\chi \mapsto B\left(P, Q, \delta_{\chi}\right)$ on $X(M)_{\sigma}$ with values in $\operatorname{End}_{\mathbb{C}}\left(E^{\prime}(\delta, H)\right)$ such that one has the equality of rational maps on $X(M)_{\sigma}$ :

$$
\xi\left(Q, \delta_{\chi}, \eta\right) \circ A\left(Q, P, \delta_{\chi}\right)=\xi\left(P, \sigma, B\left(P, Q, \delta_{\chi}\right) \eta\right), \eta \in E^{\prime}(\delta, H) .
$$

More precisely, let $b_{A}$ (resp., $\left.b_{\xi}\right)$ be a nonzero polynomial function on $X(M)_{\sigma}$, such that for all $v$ in $i_{K \cap P}^{K} E, \chi \mapsto b_{A}(\chi)\left(A\left(Q, P, \delta_{\chi}\right) v\right)$ (resp., and for all $\eta \in E^{\prime}(\delta, H)$, $\left.\chi \mapsto b_{\xi}(\chi)\left\langle\xi\left(Q, \delta_{\chi}, \eta\right), v_{\chi}\right\rangle\right)$ is polynomial on $X(M)_{\sigma}$. Then for all $\eta \in E^{\prime}(\delta, H)$, the map $\chi \mapsto\left(b_{A} b_{\xi}(\chi)\right) B\left(P, Q, \sigma_{\chi}\right) \eta$ is a polynomial map on $X(M)_{\sigma}$ with values in $E^{\prime}(\delta, H)$.

Proof. From Proposition 6.1 for $\chi$ an element of a dense open subset, $O$, of $X(M)_{\sigma}$, there is a unique $\theta(\chi) \in E^{\prime}(\delta, H)$, such that

$$
b_{\xi}(\chi) \xi\left(Q, \delta_{\chi}, \eta\right) \circ\left(b_{A}(\chi) A\left(Q, P, \delta_{\chi}\right)\right)=\xi\left(P, \delta_{\chi}, \theta(\chi)\right) .
$$

Let us show that the map $\chi \mapsto \theta(\chi)$ is polynomial in $\chi \in X(M)_{\sigma}$. Let $\theta(\chi)_{x}, x \in$ $\mathcal{W}_{M}^{G}$, be the components of $\theta(\chi)$. First, let us prove:

$$
\text { For all } e \in E,\left\langle\theta(\chi)_{1}, e\right\rangle \text { is polynomial in } \chi \in X(M)_{\sigma} \text {. }
$$

Let $J$ be a compact open subgroup of $K$ which has a $\sigma$-factorization for $\left(P, P^{-}\right)$ and such that $e$ is fixed by $J_{M}$. Then one has from (3.21)

$$
\begin{gathered}
\left\langle\theta(\chi)_{1}, e\right\rangle=\operatorname{Vol}\left((K \cap P) J_{H}\right)^{-1}\left\langle\xi\left(Q, \delta_{\chi}, \theta(\chi)\right), v_{e, \delta_{\chi}}^{P, J}\right\rangle \\
=\operatorname{Vol}\left((K \cap P) J_{H}\right)^{-1} b_{\xi}(\chi)\left\langle\xi\left(Q, \delta_{\chi}, \eta\right), b_{A}(\chi) A\left(Q, P, \delta_{\chi}\right) v_{e, \delta_{\chi}}^{P, J}\right\rangle .
\end{gathered}
$$

It follows from (3.11) that the restriction to $K$ of $v_{e, \delta_{\chi}}^{P, J}$ is independent of $\chi$. Hence, from the properties of $b_{A}$, one sees that $\chi \rightarrow v(\chi):=b_{A}(\chi) A\left(Q, P, \delta_{\chi}\right) v_{e, \delta_{\chi}}^{P, J}$ is polynomial in the compact realization. Hence (6.4) follows. 
Let $x \in \mathcal{W}_{M}^{G}$. One applies (6.4) to $\left(i_{P}^{G} \delta_{\chi}\right)^{\prime}(x) \xi\left(P, \delta_{\chi}, \eta\right)$ by changing $\sigma$ to $\sigma_{x}$. One concludes that the map $\chi \rightarrow \theta(\chi)$ is polynomial in $\chi \in X(M)_{\sigma}$. Then

$$
B\left(Q, P, \sigma_{\chi}\right) \eta:=\left(b_{\xi} b_{A}\right)^{-1}(\chi) \theta(\chi)
$$

satisfies the required properties.

\section{MAIN THEOREMS}

7.1. Let us prove

Let $P=M U$ be a $\sigma$-parabolic subgroup of $G$ and let $A_{\sigma}$ be a maximal $\sigma$-split torus of $M$. If $x, x^{\prime} \in G$ are $A_{\sigma}$-good and $P x H=P x^{\prime} H$, then $x^{\prime}=m x h$ with $m \in M, h \in H$

One has $x^{\prime}=$ muxh with $m \in M, u \in U, h \in H$. As $x^{\prime}$ is $A_{\sigma}$-good, $x^{\prime-1} . M$ is $\sigma$ stable, which implies that $x^{-1} \cdot u^{-1} . M$ is $\sigma$-stable. Let $M^{\prime}=x^{-1} . M$, which contains $x^{-1} A_{\sigma}$. Then $M^{\prime}$ is the $\sigma$-stable Levi subgroup of the $\sigma$-parabolic subgroup $P^{\prime}:=$ $x^{-1} . P$ (cf. Lemma 2.2). Hence, as $x^{-1} \cdot\left(u^{-1} \cdot M\right) \subset P^{\prime}$, one has $x^{-1} \cdot\left(u^{-1} \cdot M\right)=$ $x^{-1} . M$, which implies that $u^{-1} . M=M$. Hence, for all $m \in M, u^{-1} m u \in M$, which implies $m^{-1} u^{-1} m u \in M$. Then $m^{-1} u^{-1} m u$ is an element of $U \cap M$ and hence is equal to 1 . Hence $u$ commutes with every element of $M$. This is possible only if $u=1$. Hence $x^{\prime}=m x h$, which proves (7.1).

Lemma 7.1. (i) Let $P=M U, R=L V \subset P$ be two $\sigma$-parabolic subgroups of $G$. Let $Q$ be equal to $R \cap M$, which is a $\sigma$-parabolic subgroup of $M$ with a $\sigma$-stable Levi subgroup equal to $L$. Let $A_{\sigma}$ be a maximal $\sigma$-split torus of $M$. If $x \in M$ and $\Omega=Q x(M \cap H)$ is open in $M$, then $R x H$ is open in $G$.

(ii) Let $x, x^{\prime} \in M$, which are $A_{\sigma}$-good. If $R x H=R x^{\prime} H$, one has $Q x(M \cap H)=$ $Q x^{\prime}(M \cap H)$.

(iii) Let $(\delta, E)$ be a smooth representation of $L$ and let $\xi$ be an $H$-form on $i_{R}^{G} E$. If $\operatorname{Supp}(\xi) \subset G$ has an empty interior, the same is true for the support of $\check{r}_{M} \xi \in$ $\left(i_{Q}^{M} E\right)^{\prime}$.

Proof. (i) The first claim on $Q$ is clear as $Q \cap \sigma(Q)=R \cap \sigma(R) \cap M \cap \sigma(M)=L$.

The map $P \times H \rightarrow P H,(p, h) \mapsto p h$, is open (cf. [3. Lemma 3.1]) and $U \Omega$ is open in $P$. Hence $U \Omega H$ is open in $P H$ and also in $G$, as $P H$ is open in $G$. But $U \Omega H \subset R x H$. It implies that $R x H$ has a nonempty interior, and hence is open.

(ii) By (7.1) applied to $R$, one has $x^{\prime}=l x h$ with $l \in L, h \in H$. Hence $h$ is an element of $M \cap H$ and (ii) follows.

(iii) Let us prove that with our hypothesis, 1 does not belong to the support of $\check{r}_{M} \xi$. The hypothesis implies that $\check{r_{L}} \xi=0$. From Proposition 3.2 (iii), one deduces that $\left.\check{r}_{L} \xi=\check{r}_{L}\left(\check{r}_{M} \xi\right)\right)=0$, which is equivalent to the fact that 1 is not in the support of $\check{r}_{M} \xi$. This proves our claim.

Now let $x$ be as in (i). Changing the representative in $Q x(H \cap H)$, we may assume (cf (2.22) ) that $x$ is $A_{\sigma}$-good . Then $P$ is a $\sigma_{x}$-parabolic subgroup of $G$ (cf. Lemma 2.2). Then one applies the first part of the proof to $\left(i_{R}^{G} \delta\right)^{\prime}(x) \xi$, which is fixed by $x . H$, replacing $\sigma$ by $\sigma_{x}$. This implies that $x$ is not an element of the support of $\check{r}_{M} \xi$.

Lemma 7.2. Let $P=M U, R=L V$ be two $\sigma$-parabolic subgroups of $G$ with $R \subset P$. Let $Q=M \cap R$, which is a $\sigma$-parabolic subgroup of $M$. Let $A$ be a $\sigma$-stable maximally $\sigma$-split maximal split torus of $L$. We choose the set $\mathcal{W}_{M}^{G}$ such that its elements are $A_{\sigma}$-good. Then for all $y \in \mathcal{W}_{M}^{G}, y^{-1} . M$ is $\sigma$-stable and $y^{-1}$. $Q$ is a 
$\sigma$-parabolic subgroup of $y^{-1} . M$ with a $\sigma$-stable Levi subgroup equal to $y^{-1}$.L. For all $y \in \mathcal{W}_{M}^{G}$, we choose $\mathcal{W}_{y^{-1} . L}^{y^{-1}} \cdot M$ such that its elements are $y^{-1} \cdot A_{\sigma}$-good. Then $\mathcal{W}:=\bigcup_{y \in \mathcal{W}_{M}^{G}} y \mathcal{W}_{y^{-1} . L}^{y^{-1} \cdot M}$ is made of $A_{\sigma}$-good elements, the union being disjoint, and may be taken as $\mathcal{W}_{L}^{G}$.

Proof. We will show that $\mathcal{W}$ is a set of representatives of the open $(R, H)$-double cosets in $G$.

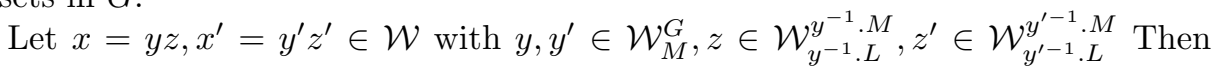
$x, x^{\prime}$ are $A_{\sigma}$-good. Hence $R x H$ and $R x^{\prime} H$ are open. Moreover $P x H=P y H$, $P x^{\prime} H=P y^{\prime} H$ as $z \in y^{-1} . M, z^{\prime} \in y^{\prime-1} . M$. Let us show that:

The equality $R x H=R x^{\prime} H$ implies $y=y^{\prime}$ and $z=z^{\prime}$.

Our hypothesis implies $P x H=P x^{\prime} H$. By what we have just seen, it implies $P y H=P y^{\prime} H$. Hence one has $y=y^{\prime}$.

First, let us assume that $y=y^{\prime}=1$. Then $z, z^{\prime} \in M$ are $A_{\sigma}$-good, $R z H=R z^{\prime} H$, and, by (7.1), $z^{\prime}=l z h$, where $l \in L$ and $h \in H$. Hence one has $h \in M \cap H$. If $Q=R \cap M, z, z^{\prime}$ determine the same $(Q, M \cap H)$-double coset. This implies that $z^{\prime}=z$. Hence this proves (7.2) if $y=y^{\prime}=1$.

For the general case, we apply this to $y^{-1} \cdot L$ and $y^{-1} \cdot R$ in order to prove (7.2). So $\mathcal{W}$ is a set of representatives of certain open $(R, H)$-cosets.

Reciprocally, let $R x H$ be an open $(R, H)$-double coset in $G$. We may assume that $x$ is $A_{\sigma}$-good. Let $Q=R \cap M$. Let us show that there is $x^{\prime} \in \mathcal{W}$ with $R x H=R x^{\prime} H$. First there exists $y \in \mathcal{W}_{M}^{G}$ such that $P x H=P y H$. Let us assume that $y=1$. Then, by (7.1), one has $x=m h$ with $m \in M, h \in H$. Hence one can assume $x \in M$. As $x \in M$ is $A_{\sigma}$-good, $Q x(H \cap M)$ is open in $M$ and $Q x(H \cap M)=Q x^{\prime}(H \cap M)$ with $x^{\prime} \in \mathcal{W}_{L}^{M}$. Then $R x H=R x^{\prime} H$, as wanted. In general one changes $P$ to $P^{\prime}=y^{-1} \cdot P$ and $R$ to $R^{\prime}=y^{-1} \cdot R$. Hence one has $P^{\prime} y^{-1} x H=P^{\prime} H$, as $P x H=P y H$. Then one uses our last result.

\section{2. $\sigma$-exponents of $j_{P^{-}} \xi$.}

Definition 7.3. Let $(\pi, V)$ be a smooth representation of $G$ of finite length. Then it is a finite direct sum of generalized eigenspaces under $A_{G, \sigma}:=\left(A_{G}\right)_{\sigma}$. If $\nu$ is a character of $A_{G, \sigma}$, let us denote the corresponding generalized eigenspace of $V$ by $V(\nu)$ and the restriction to $V(\nu)$ of any element $\xi$ of $V^{\prime}$ by $\xi(\nu)$, which might be extended to an element of $V^{\prime}$, which is zero on the other generalized eigenspaces also denoted $\xi(\nu)$. If $\xi \in V^{\prime H}, \operatorname{Exp}(\xi)$ will denote the subset of $\nu$ such that $\xi(\nu)$ is nonzero. The elements of $\operatorname{Exp}(\xi)$ are called the $A_{G, \sigma}$-exponents or $\sigma$-exponents of $\xi$.

Theorem 7.4. Let $P=M U$ (resp., $P^{\prime}=M^{\prime} U^{\prime}$ ) be a $\sigma$-parabolic subgroup of $G$ and let $A$ (resp., $\left.A^{\prime}\right)$ be a maximally $\sigma$-split $\sigma$-stable maximal split torus of $M$ (resp., $\left.M^{\prime}\right)$. Let $\left(P^{\prime} \backslash G / P\right)_{\sigma}$ be the set of $\left(P^{\prime}, P\right)$-double cosets in $G$ having a representative $w$ such that $w . A=A^{\prime}, w \cdot A_{\sigma}=A_{\sigma}^{\prime}$. We denote a set of representatives of $\left(P^{\prime} \backslash G / P\right)_{\sigma}$ with this property by $W\left(M^{\prime} \backslash G / M\right)_{\sigma}$ and we assume that the set $W\left(M^{\prime} \backslash G / M\right)$ defined in (4.10) contains $W\left(M^{\prime} \backslash G / M\right)_{\sigma}$.

Let $(\delta, E)$ be an irreducible smooth represention of $M$ and let $\eta$ be an element of the space $E^{\prime}(\delta, H)$ (cf. (6.1) for the definition of this space). Let $O^{\prime}$ be as in Theorem 4.11, with $X=X(M)_{\sigma}$. In particular it is open and dense in $X(M)_{\sigma}$. Let $O_{0}$ be the 
open dense subset of $X(M)_{\sigma}$ from Proposition 6.1 (ii) for $X=X(M)_{\sigma}$. Let $\chi$ be an element of the open and dense subset $O^{\prime} \cap O_{0}$ of $X(M)_{\sigma}$ and let $\xi=\xi\left(P, \delta_{\chi}, \eta\right)$.

(i) Let $w \in W\left(M^{\prime} \backslash G / M\right)$. Then if $\xi_{P^{\prime-}, w}$ is nonzero, one has $w \in W\left(M^{\prime} \backslash G / M\right)_{\sigma}$.

(ii) If $w \in W\left(M^{\prime} \backslash G / M\right)_{\sigma}$, then $Q=M \cap w^{-1} \cdot P^{\prime}$ is a $\sigma$-parabolic subgroup of $M$ and $L=M \cap w^{-1} . M^{\prime}$ is its $\sigma$-stable Levi subgroup. We introduce $\mathcal{W}_{L}^{G}$ as in the preceding lemma.

If $y \in \mathcal{W}_{M}^{G}$ and $z \in \mathcal{W}_{y^{-1} . L}^{y^{-1} . M}$, we define $z^{\prime}:=y z y^{-1} \in M$ and $x=y z$. Then $\delta^{\prime}\left(z^{\prime}\right) \eta_{y}$ is $M \cap x . H$-invariant and $Q$ is a $\sigma_{x}$-parabolic subgroup of $M$. Hence $j_{Q^{-}} \delta^{\prime}\left(z^{\prime}\right) \eta_{y}$ is defined.

(iii) One writes

$$
E_{Q}=E_{Q}^{+} \oplus E_{Q}^{0},
$$

where $E_{Q}^{+}$is the sum over $y \in \mathcal{W}_{M}^{G}$ and $z \in \mathcal{W}_{y^{-1} . L}^{y^{-1} . M}$ of the $A_{L, \sigma}$-weight space corresponding to the set of exponents of $j_{Q^{-}} \delta^{\prime}\left(z^{\prime}\right) \eta_{y}$, where $E_{Q}^{0}$ is the sum over the other weights.

Then, for $\chi$ belonging to an open dense subset, $O^{\prime \prime}$ of $O_{0} \cap O^{\prime}$, hence also open and dense in $X(M)_{\sigma}$, the $A_{M^{\prime}, \sigma^{-}}$exponents of $\xi_{P^{\prime-}, w}$ are of the form $\left(w \chi^{+}\right)_{\mid A_{M^{\prime}, \sigma}}$ where $\chi^{+}$is an $A_{M^{\prime}, \sigma}$-eigenvalue of $\left(E_{Q}^{+}\right)_{\chi}$.

Proof. (i) If $\xi_{P^{\prime-}, w}$ is nonzero, one has $w \in W\left(M^{\prime} \backslash G / M\right)_{\sigma}$ by Proposition 5.3 . This proves (i).

Let us prove (ii). From (i), $w^{-1} \cdot P^{\prime}$ is a $\sigma$-parabolic subgroup of $G$, as $w$ is $A_{\sigma}^{\prime}$-good, which contains $A$. Hence $Q$ is a $\sigma$-parabolic subgroup of $M$.

Let $x, y, z$ be as in statement (ii). The linear form $\delta^{\prime}\left(z^{\prime}\right) \eta_{y}$ on $E$ is $M \cap x . H$ invariant as $\eta_{y}$ is $M \cap y$. $H$-invariant and $z^{\prime} y=y z=x$. By the construction of $\mathcal{W}_{M}^{G}$, $x$ is $A_{\sigma}$-good. Hence $Q, Q^{-}$are opposite $\sigma_{x}$-parabolic subgroups of $M$ (cf. Lemma 2.2). This proves (ii).

Let us prove (iii). One defines projections $p_{Q}^{+}$and $p_{Q}^{0}$ of $E_{Q}$ onto $E_{Q}^{+}$and $E_{Q}^{0}$ corresponding to the decomposition $E_{Q}=E_{Q}^{+} \oplus E_{Q}^{0}$. This defines, by induction, projections on the space $\left(i_{P_{Q^{-}}}^{G}\left(E_{\chi}\right)_{Q}\right)^{\prime}$ that we will denote in the same way. Notice that $P_{Q^{-}}$is equal to $\tilde{P}_{w}$. With these conventions, we define for $\chi \in O_{0} \cap O^{\prime}$ :

$$
\xi_{Q}^{+}=p_{Q}^{+}\left(\check{j}_{Q^{-}} \circ \xi\right) .
$$

Similarly one defines $\xi_{Q}^{0}$. Then

$$
\xi_{P^{\prime-}, w}^{+}:=\check{r}_{M^{\prime}} \circ{ }^{t} A\left(w \cdot \tilde{P}_{w}, \tilde{P}_{w}^{\prime}, w \cdot j_{Q} \delta_{\chi}\right) \circ \lambda(w) \xi_{Q}^{+}
$$

is a well-defined element of $V_{w}^{\prime}$ where $V_{w}:=i_{M^{\prime} \cap w . P}^{M^{\prime}}\left(w E_{\chi}^{+}\right)$. Similarly one defines $\xi_{P^{\prime-}, w}^{0}$ so that one has:

$$
\xi_{P^{\prime-}, w}=\xi_{P^{\prime-}, w}^{+}+\xi_{P^{\prime-}, w}^{0} .
$$

We will prove that $\xi_{P^{\prime-}, w}^{0}=0$. We first study the restriction of $\xi_{Q}^{0}$ on the open $\left(M^{\prime} \cap w . P, M^{\prime} \cap H\right)$-orbits. From Lemma 3.4, one sees that for $x \in \mathcal{W}_{L}^{G}$ :

$$
\check{r}_{L}\left(\left(i_{P_{Q^{-}}}^{G}\left(\delta_{\chi}\right)_{Q}\right)^{\prime}(x) \check{j}_{Q^{-}} \circ \xi\right)=j_{Q^{-}}\left(\check{r}_{M}\left(\left(i_{P}^{G} \delta_{\chi}\right)^{\prime}(x) \xi\right)\right) .
$$

But if $x=y z$ with $y \in \mathcal{W}_{M}^{G}$ and $z \in \mathcal{W}_{y^{-1} . L}^{y^{-1} \cdot M}$, one has $x=z^{\prime} y$ with $z^{\prime}=y z y^{-1} \in M$ and, with the notation of (3.20), one has:

$$
\check{r}_{M}\left(\left(i_{P}^{G} \delta_{\chi}\right)^{\prime}(x) \xi\right)=f_{\xi}(x)=\delta_{\chi}^{\prime}\left(z^{\prime}\right) f_{\xi}(y)=\chi\left(z^{\prime}\right)^{-1} \delta^{\prime}\left(z^{\prime}\right) \eta_{y} .
$$


Hence, one has:

$$
\check{r}_{L}\left(\left(i_{P_{Q^{-}}}^{G}\left(\delta_{\chi}\right)_{Q}\right)^{\prime}(x) \check{j}_{Q^{-}} \circ \xi\right)=\chi\left(z^{\prime}\right)^{-1} j_{Q^{-}}\left(\delta^{\prime}\left(z^{\prime}\right) \eta_{y}\right)
$$

and the result is not changed if one replaces $\check{j}_{Q} \circ \xi$ by $\xi_{Q}^{+}$. This implies:

$$
\left.\check{r}_{L}\left(i_{P_{Q^{-}}}^{G}\left(\delta_{\chi}\right)_{Q}\right)^{\prime}(x) \xi_{Q}^{0}\right)=0 .
$$

From the preceding lemma, one sees that the support of $\xi_{Q}^{0}$ has an empty interior. By structural transport the same is true for $\lambda(w) \circ \xi_{Q}^{0}$. One can apply Lemma 5.5 with $L$ replaced by $w \cdot M$ and $M$ replaced by $M^{\prime} \cap w \cdot M$ as it follows immediately from the definition of $\tilde{P}_{w}, \tilde{P}_{w}^{\prime}$ in Proposition 4.9. Then by Lemma 5.5 and Lemma 7.1, one sees that the support of $\xi_{P^{\prime-}, w}^{0}$ has an empty interior. Let us see that one can apply Proposition 6.1 to $M^{\prime}, M^{\prime} \cap w \cdot P, w \cdot\left(A_{M}\right)_{\sigma}$ and $X^{\prime}=\left\{(w \chi)_{\mid M^{\prime} \cap w . M} \mid \chi \in\right.$ $\left.X(M)_{\sigma}\right\}$ instead of $G, P, B$ and $X$. As $w A_{\sigma}=A_{\sigma}^{\prime}$, one sees that $M^{\prime} \cap w . P$ is a $\sigma$-parabolic subgroup of $w \cdot M$ with $M^{\prime} \cap w \cdot M$ as its $\sigma$-stable Levi subgroup. Then $X^{\prime} \subset X\left(M^{\prime} \cap w \cdot M\right)_{\sigma}$ and its Lie algebra is equal to $w\left(\mathfrak{a}_{M}^{\prime}\right)_{\mathbb{C}}$, and if $\lambda$ is any strictly $P$-dominant element in $\Lambda\left(A_{M}\right)_{\sigma}, w \cdot \lambda$ is a strictly- $\left(M^{\prime} \cap w \cdot P\right)$ dominant of $\Lambda\left(w \cdot\left(A_{M}\right)_{\sigma}\right.$. Then Proposition 6.1 implies that $\xi_{P^{\prime-}, w}^{0}$ is equal to zero for $\chi$ belonging to an open dense subset, $O^{\prime \prime}$ of $O_{0} \cap O^{\prime}$. Hence one has:

$$
\xi_{P^{\prime-}, w}=\xi_{P^{\prime-}, w}^{+} .
$$

As $\xi_{P^{\prime-}, w}^{+}$is a linear form on $i_{M^{\prime} \cap w . P}^{M^{\prime}} w\left(\left(E_{Q}^{+}\right)_{\chi_{\mid M \cap w^{-1} M^{\prime}}}\right)$, (iii) follows.

An $H$-form $\xi$ on a smooth admissible representation of $G,(\pi, V)$ is said to be $H$-cuspidal if $j_{Q^{-}} \xi=0$ for all proper $\sigma$-parabolic subgroups of $G$. We denote the space of cuspidal $H$-forms on $V$ by $V_{c u s p}^{\prime H}$.

We define

$$
E^{\prime}(\delta, H)_{c u s p}:=\bigoplus_{x \in \mathcal{W}_{M}^{G}}\left(E^{\prime}\right)_{c u s p}^{M \cap x . H} .
$$

Theorem 7.5. Let $\eta \in E^{\prime}(\delta, H)_{\text {cusp. }}$. Let $w \in W\left(M^{\prime} \backslash G / M\right)_{\sigma}$. With the notation of the preceding theorem, let $\chi$ be an element of the open and dense subset $O^{\prime \prime}$ of $X(M)_{\sigma}$ and let $\xi=\xi\left(P, \delta_{\chi}, \eta\right)$.

(i) If $\xi_{P^{\prime-}, w} \neq 0$, one has $M \cap w^{-1} \cdot P^{\prime}=M$.

(ii) If $M \cap w^{-1} \cdot P^{\prime}=M$, one defines $\mathcal{W}_{w . M}^{G}:=w \mathcal{W}_{M}^{G}$ whose elements are $A_{\sigma}^{\prime}=$ $w . A_{\sigma}=A_{\sigma}^{\prime}$-good. One can choose $\mathcal{W}_{w . M}^{M^{\prime}}$ such that for $y^{\prime} \in \mathcal{W}_{w . M}^{M^{\prime}}$, there exists a unique $y \in \mathcal{W}_{w . M}^{G}$ and $h \in M^{\prime} \cap H$ with $y^{\prime}=y h$.

If $\eta \in E^{\prime}(w \delta, H), p_{M^{\prime}} \eta:=\left(\eta_{y}\right)_{y^{\prime} \in \mathcal{W}_{w, M}^{M^{\prime}}}$ is an element of $(w E)^{\prime}\left(w \delta, M^{\prime} \cap H\right)$. With this notation,

$$
\xi_{P^{\prime-}, w}=\xi\left(M^{\prime} \cap w \cdot P, w \delta_{\chi}, p_{M^{\prime}} B\left(\tilde{P}_{w}^{\prime}, w \cdot P, w \delta_{\chi}\right) \eta\right),
$$

where the $B$-matrices are defined relative to $\mathcal{W}_{w . M}^{G}$.

(iii) Let us assume that $M^{\prime}=M$ and $A^{\prime}=A$. Then $B\left(P^{\prime}, P, \delta_{\chi}\right) \eta$ is an element of $E^{\prime}(\delta, H)_{\text {cusp }}$. Hence $B\left(P^{\prime}, P, \delta_{\chi}\right)$ restricts to an endomorphism of $E^{\prime}(\delta, H)_{\text {cusp }}$.

Proof. (i) Let us assume that $M \cap w^{-1} P^{\prime} \neq M$. From the definition of $E^{\prime}(\delta, H)_{\text {cusp }}$ and of $E_{Q}^{+}$in Theorem 7.4 (iii) one will see that $E_{Q}^{+}$is zero. In fact, with the notation of Theorem 7.4 (ii), as $\eta_{y}$ is $M \cap y . H$ cuspidal, one sees by a direct computation that $\delta^{\prime}\left(z^{\prime}\right) \eta_{y}$ is $M \cap x . H$-cuspidal and $j_{Q^{-}} \delta^{\prime}\left(z^{\prime}\right) \eta_{y}=0$. This implies that $E_{Q}^{+}=\{0\}$. From the preceding theorem one has $\xi_{P^{\prime-}, w}=0$. 
(ii) Let us assume $M \cap w^{-1} \cdot P^{\prime}=M$. Then the $\sigma$-stable Levi subgroup of $P_{w}^{\prime}$ is equal to $w \cdot M$.

If $y^{\prime} \in M^{\prime}$ is $A_{\sigma}^{\prime}$-good, then $P_{w}^{\prime} y^{\prime} H$ is open and $P_{w}^{\prime} y^{\prime} H=P_{w}^{\prime} y H$ for some element $y$ of $\mathcal{W}_{w . M}^{G}$. In particular $y$ is $A_{\sigma}^{\prime}$-good. From (7.1), one sees that $y^{\prime}=l y h$ with $l \in M^{\prime} \cap w \cdot M, h \in H$. Changing $y^{\prime}$ to $l^{-1} y^{\prime}$, one may assume that $y^{\prime}=y h$ for some $h \in M^{\prime} \cap H$. This allows us to choose $\mathcal{W}_{w . M}^{M^{\prime}}$ as in (ii). From Theorem 4.11

$$
\xi_{P^{\prime-}, w}=\check{r}_{M^{\prime}}{ }^{t} A\left(w \cdot P, \tilde{P}_{w}^{\prime}, w\left(\delta_{\chi}\right)\right) \lambda(w) \xi .
$$

One has chosen $\mathcal{W}_{w . M}^{G}:=w \mathcal{W}_{M}^{G}$ whose elements are $A_{\sigma}^{\prime}=w \cdot A_{\sigma}$-good. Then, one sees that $(w E)^{\prime}(w \delta, H)_{c u s p}=E^{\prime}(\delta, H)_{c u s p}$, as $\left(E^{\prime}\right)_{\text {cusp }}^{M \cap x . H}=\left((w E)^{\prime}\right)_{\text {cusp }}^{(w . M) \cap(w x . H)}$. Also one has $\lambda(w) \xi\left(P, \delta_{\chi}, \eta\right)=\xi\left(w \cdot P, w\left(\delta_{\chi}\right), \eta\right)$. Hence from the definition of $B$ matrices one has:

$$
\xi_{P^{\prime-}, w}=\check{r}_{M^{\prime}} \xi\left(\tilde{P}_{w}^{\prime}, w\left(\delta_{\chi}\right), B\left(\tilde{P}_{w}^{\prime}, w \cdot P, w\left(\delta_{\chi}\right)\right) \eta\right) .
$$

In order to prove the equality of the theorem for $\chi$ in an open dense subset of $X(M)_{\sigma}$, one can use Proposition 6.1 applied to $M^{\prime}$ instead of $G$ and $M^{\prime} \cap w \cdot P^{\prime}$ instead of $P$ to the complex torus $X=\left\{w \chi_{\mid M^{\prime} \cap w \cdot M} \mid \chi \in X(M)_{\sigma}\right\}$ and to $B=$ $w \cdot\left(A_{M}\right)_{\sigma}$. Then it suffices to prove the equality of the values of $\check{r}_{M^{\prime}} \xi\left(P_{w}^{\prime}, w\left(\delta_{\chi}\right)\right.$, $\left.B\left(P_{w}^{\prime}, w \cdot P, w\left(\delta_{\chi}\right) \eta\right)\right)$ and $\xi\left(M^{\prime} \cap w \cdot P, w \delta_{\chi}, p_{M^{\prime}} B\left(P_{w}^{\prime}, w \cdot P, w \delta_{\chi}\right), \eta\right)$ at every element, $y^{\prime}$, of $\mathcal{W}_{w . M}^{M^{\prime}}$. This is easily seen from (6.3) and from the fact that $y^{\prime}=y h$ for an element $y$ of $\mathcal{W}_{w . M}^{G}$ and $h \in H \cap M^{\prime}$.

(iii) We take $w=1$ in (ii). Hence, one has:

$$
\xi_{P^{\prime-}, 1}=B\left(P^{\prime}, P, \delta_{\chi}\right) \eta \in V(\delta, H) .
$$

From (i) and the transitivity of the constant term, one sees that $\xi_{P^{\prime-}, 1} \in E^{\prime}(\delta, H)_{\text {cusp }}$ is $M \cap H$-cuspidal.

If $P=M U$ is a $\sigma$-parabolic subgroup of $G$, let us denote $\left(A_{M}\right)_{\sigma}$ by $A_{M, \sigma}$. Let ${ }^{+} \mathfrak{a}_{M, \sigma}^{\prime}\left(\operatorname{resp}{ }^{++} \mathfrak{a}_{M, \sigma}^{\prime}\right)$ be the set of $\lambda \in \mathfrak{a}_{M, \sigma}^{\prime}$ which are linear combinations of roots of $A_{M, \sigma}$ in the Lie algebra of $U$ with coefficients greater than or equal to zero (resp., greater than zero).

Definition 7.6. Let $(\pi, V)$ be a finite length smooth representation of $G$ and $\xi$ an $H$-form on $V$. Then $\xi$ is said to be tempered (resp., discrete) if and only if for every $\sigma$-parabolic subgroup of $G, P=M U$, every exponent, $\chi$, of $\xi_{P^{-}}$is such that $\operatorname{Re}(\chi)$ is an element of ${ }^{+} \mathfrak{a}_{M, \sigma}^{\prime}\left(\operatorname{resp}{ }^{++} \mathfrak{a}_{M, \sigma}^{\prime}\right)$. If $\xi$ is a tempered $H$-form we define its weak constant term

$$
\xi_{P^{-}}^{w}=\sum_{\chi \in \operatorname{Exp}\left(\xi_{P^{-}}\right), \operatorname{Re}(\chi)=0} \xi_{P^{-}}(\chi) .
$$

Hence a discrete $H$-form is a tempered $H$-form such that its weak constant term is zero for all proper $\sigma$-parabolic subgroups of $G$. Notice that Kato-Takano (cf. [21]) showed that, if $\pi$ is irreducible and has a unitary central character, an $H$-form is discrete if and only if its generalized coefficients are square integrable modulo the center.

Lemma 7.7. (i) If $\xi$ is a tempered $H$-form on $V$, then $\xi_{P^{-}}^{w}$ is a tempered $M \cap H$ form on $V_{P}$.

(ii) If $Q$ is a $\sigma$-parabolic subgroup of $M$ and $R=Q U$, one has:

$$
\xi_{R^{-}}^{w}=\left(\xi_{P^{-}}^{w}\right)_{Q^{-}}^{w} .
$$


Proof. (i) and (ii) follow from the transitivity of the constant term (cf. [12]).

If $(\delta, E)$ is a smooth unitary irreducible representation of $M$, let $E_{d i s c}^{\prime M \cap H}$ be the space of discrete $M \cap H$-forms on $E$ and let:

$$
E^{\prime}(\delta, H)_{d i s c}=\bigoplus_{x \in \mathcal{W}_{M}^{G}} E_{d i s c}^{\prime M \cap x . H} .
$$

Theorem 7.8. With the notation of Theorem 7.4, let us assume $\delta$ is unitary, $\eta \in E^{\prime}(\delta, H)_{\text {disc }}$ and $\chi \in O^{\prime \prime} \cap X(M)_{u}$. Then one has:

(i) The $H$-form $\xi\left(P, \delta_{\chi}, \eta\right)$ is tempered.

(ii) If $w \in W\left(M^{\prime} \backslash G / M\right)$ is not in $W\left(M^{\prime} \backslash G / M\right)_{\sigma}$ or if $M \cap w^{-1} . P^{\prime}$ is distinct from $M$, then $\xi_{P^{\prime-}, w}^{w}=0$. Otherwise, with the notation of Theorem 7.5 (ii):

$$
\xi_{P^{\prime-}, w}^{w}=\xi\left(M^{\prime} \cap w \cdot P, w \delta_{\chi}, p_{M^{\prime}} B\left(\tilde{P}_{w}^{\prime}, w \cdot P, w \delta_{\chi}\right) \eta\right),
$$

where the $B$-matrices are defined relative to $\mathcal{W}_{w . M}^{G}$.

(iii) Let us assume that $M^{\prime}=M$ and $A^{\prime}=A$. Then $B\left(P^{\prime}, P, \delta_{\chi}\right)$ restricts to an endomorphism of $E^{\prime}(\delta, H)_{d i s c}$.

Proof. Let us use the notation of Theorem 7.4 (ii). Using the criteria of Kato and Takano (see above), one sees by "transport de structure" that $\delta^{\prime}\left(z^{\prime}\right) \eta_{y}$ is discrete. Moreover $Q$ is a $\sigma_{x}$-parabolic subgroup of $M$. Then (i) follows from Theorem 7.4 (iii) and from our definition of discrete forms.

The proof of (ii) (resp., (iii)) is analogous to the proof of (i) and (ii) (resp., (iii)) of the preceding theorem.

\section{Constant term of Eisenstein integrals}

If $f$ is a smooth function on $H \backslash G$ the constant term $f_{P}$ of $f$ along $P$ has been defined in [12, Section 3.3]. It generalizes the definition in [23, Proposition 2] to general smooth function. It is a smooth function on $M \cap H \backslash M$.

If $(\pi, V)$ is a smooth admissible representation of $G, \xi$ is an $H$-form on $V$ and $v$ is an element of $V$, let $c_{\xi, v}$ be the generalized coefficient defined by:

$$
c_{\xi, v}(H g)=\langle\xi, \pi(g) v\rangle .
$$

Let $P=M U$ be a $\sigma$-parabolic subgroup of $G$. If $f=c_{\xi, v}$, the constant term $f_{P}$ of $f$ along $P$ has been defined in [23, Proposition 2 (i)] (see [12, Section 3.3], for a definition for general smooth functions). Then (cf. [23, Proposition 2 (ii)]) one has:

$$
\left(c_{\xi, v}\right)_{P}=c_{\xi_{P^{-}}, v_{P}},
$$

where $\xi_{P^{-}}=j_{P^{-}} \xi, v_{P}=j_{P} v$.

Definition 8.1. We define $\mathcal{A}_{\text {temp }}(H \backslash G)$ to be the set of functions of the type $c_{\xi, v}$ for a finite length smooth representation $(\pi, V)$ of $G$ and a tempered $H$-form on $V$. It is easily seen to be a vector subspace of the space of smooth functions on $H \backslash G$. If $f$ is such a generalized coefficient $f_{P}^{w}$ will denote the generalized coefficient $c_{\xi_{P-}^{w}, v_{P}}$. It is naturally deduced from the constant term $f_{P}$ as in the definition of $\xi_{P^{-}}^{w}$ (cf. Definition (7.6), hence it does not depend on the presentation of $f$ as a generalized coefficient. 
Definition 8.2. Let $P=M U$ be a $\sigma$-parabolic subgroup of $G$ and let $(\delta, E)$ be an irreducible smooth representation of $M$. Let $\eta \in E^{\prime}(\delta, H)$ be such that $\xi(P, \delta, \eta)$ is defined. Then if $v \in i_{P}^{G} E$, one defines an element $E_{P}^{G}(\eta \otimes v)$ of $C^{\infty}(H \backslash G)$ by:

$$
E_{P}^{G}(\eta \otimes v)(g)=\left\langle\xi(P, \delta, \eta),\left(i_{P}^{G} \delta\right)(g) v\right\rangle, g \in G .
$$

Then, from Proposition 6.1, there exists a nonzero polynomial function $b$ on $X(M)_{\sigma}$ such that for all $v \in i_{K \cap P}^{K} E, \eta \in E^{\prime}(\delta, H), g \in G$ the map $\chi \mapsto b(\chi) E_{P}^{G}\left(\eta \otimes v_{\chi}\right)(g)$ is polynomial in $\chi \in X(M)_{\sigma}$. By bilinearity, we define $E_{P}^{G}(\phi)$ similarly, where $\phi$ is an element of $E^{\prime}(\delta, H) \otimes i_{P}^{G} E$.

Lemma 8.3. Let $W\left(M^{\prime}|G| M\right)_{\sigma}$ be the set of elements of $\left.W\left(M^{\prime} \backslash G / M\right)_{\sigma}\right)$ such that w.M $\subset M^{\prime}$. Let us assume that $W\left(M^{\prime}|G| M\right)_{\sigma}$ is non empty. Let $s \in$ $W\left(M^{\prime}|G| M\right)_{\sigma}$. We choose $K^{\prime}=s . K$ (see the beginning of Section 4.2). This choice together with the choice of $K$ determines $\beta_{\chi}$. Let $w$ be an element of $W\left(M^{\prime}|G| M\right)_{\sigma}$. Let $\gamma\left(G \mid M^{\prime}\right)$ be the constant defined in [29] after the proof of I.1.(3), with the normalization of measures determined by $K^{\prime}$. For $\chi$ belonging to an open dense subset of $X(M)_{\sigma}$ one has, with the notation of (4.19):

$$
\gamma_{\chi, w}=\gamma\left(G \mid M^{\prime}\right)^{-1} \alpha_{\chi, w}
$$

Proof. It is enough, by "transport de structure", to prove the result for $s^{-1} \cdot P^{\prime}$ instead of $P^{\prime}$, as the Jacquet modules for $P^{\prime}$ and $s^{-1} \cdot P^{\prime}$ of $i_{P}^{G} \delta_{\chi}$ are canonically isomorphic. So we may assume that $1 \in W\left(M^{\prime} \backslash G / M\right)_{\sigma}$ and $A^{\prime}=A, K^{\prime}=K$. By Lemma 4.7 applied to $M^{\prime}$ instead of $G$, one sees that for $\chi$ belonging to a dense subset $O^{\prime \prime}$ of $X(M)_{\sigma}, V_{\chi, w}$ is irreducible for all $w \in W\left(M^{\prime} \backslash G / M\right)_{\sigma}$. Hence $\gamma_{\chi, w}=\gamma(P, w, \chi) \alpha_{\chi, w}$ for an element $\gamma(P, w, \chi)$ of $\mathbb{C}^{*}$. Notice that in the definition of $\beta_{\chi, w}$ (resp. $\alpha_{\chi, w}$ ) the intertwining integrals which occur are relative to parabolic subgroups of $G$ with a Levi subgroup equal to $M$. Also only the operations $j_{M}$ and $\check{j}_{M}$ occur and they are equal to the identity. Then the proof of the lemma is identical to the proof of Proposition V.1.1 in 29] (see Equations V.1 (2), (3) and (4)), where parabolic subgroups have to be replaced by $\sigma$-parabolic subgroups.

Theorem 8.4. We keep the notation of Theorem 7.4. Let $\chi$ be an element of the dense open subset $O^{\prime \prime}$ of $X(M)_{\sigma}$. If $w \in W\left(M^{\prime}|G| M\right)_{\sigma}$, one defines a linear map $C\left(w, P^{\prime}, P, \delta_{\chi}\right)$ from $E^{\prime}(\delta, H) \otimes i_{P}^{G} E_{\chi}$ to $(w E)^{\prime}\left(w \delta, M^{\prime} \cap H\right) \otimes i_{P_{w}}^{G} w E_{\chi}$ by :

$$
C\left(w, P^{\prime}, P, \delta_{\chi}\right)=\gamma\left(G \mid M^{\prime}\right)^{-1} B\left(\tilde{P}_{w}^{\prime}, w \cdot P, w\left(\delta_{\chi}\right)\right) \otimes\left(A\left(P_{w}^{\prime}, w \cdot P, w\left(\delta_{\chi}\right)\right) \lambda(w)\right) .
$$

Then, if $\phi \in E^{\prime}(\delta, H)_{c u s p} \otimes i_{P}^{G} E_{\chi}$ (resp., $E^{\prime}(\delta, H)_{d i s c} \otimes i_{P}^{G} E_{\chi}$ and $\delta$ and $\chi$ are unitary), one has: $E_{P}^{G}(\phi)_{P^{\prime}}=0$ (resp., $\left.E_{P}^{G}(\phi)_{P^{\prime}}^{w}=0\right)$ if $W\left(M^{\prime}|G| M\right)_{\sigma}$ is empty. Otherwise $E_{P}^{G}(\phi)_{P^{\prime}}$ (resp., $\left.E_{P}^{G}(\phi)_{P^{\prime}}^{w}\right)$ is equal to:

$$
\sum_{w \in W\left(M^{\prime}|G| M\right)_{\sigma}} E_{M^{\prime} \cap w . P}^{M^{\prime}}\left(\left(p_{M^{\prime}} \otimes r_{M^{\prime}}\right)\left(C\left(w, P^{\prime}, P, \delta_{\chi}\right) \phi\right)\right) .
$$

Proof. By $M^{\prime}$-equivariance, it is enough to prove the equalities of the proposition evaluated at $m^{\prime}=1$. Then the result follows from property (8.1) (resp., Definition 8.1) and from (4.19) together with Theorem 7.5 (resp., Theorem 7.8) and Lemma 8.3 


\section{ApPENDix}

9.1. Covariant distributions on a homogeneous space. Let $X$ be an $l$-space (cf. e.g. [3, section 3] for the terminology) and let $V$ be a complex vector space. We denote the space of locally constant functions on $X$ with compact support and with values in $\mathbb{C}$ (resp., $V$ ) by $C_{c}^{\infty}(X)$ (resp., $C_{c}^{\infty}(X, V)$ ). Notice that $C_{c}^{\infty}(X, V)$ is identified with $C_{c}^{\infty}(X) \otimes V$. We denote the space of linear forms on $C_{c}^{\infty}(X, V)$ which are called $V$-distributions on $X$ by $\mathcal{D}^{\prime}(X, V)$. The support of a $V$-distribution $T$ on $X$ is the complement of the largest open subset $O$ such that $T$ restricted to $C_{c}^{\infty}(O) \otimes V$ is equal to zero.

Let $F$ be a closed subset of $X$ and let $O$ denote $X \backslash F$. From the exact sequence

$$
0 \rightarrow C_{c}^{\infty}(O) \otimes V \rightarrow C_{c}^{\infty}(X) \otimes V \rightarrow C_{c}^{\infty}(F) \otimes V \rightarrow 0,
$$

one sees that if $T$ has support contained in $F$, then $T$ defines a $V$-distribution on $F$ which is called the distribution on $F$ induced by $T$.

Let $(\pi, V)$ be a smooth representation of an $l$-group $G$. Recall that $H_{0}(G, V)$ denotes the quotient of $V$ by the subspace generated by the elements of the form $\pi(g) v-v, g \in G, v \in V$. The dual of $H_{0}(G, V)$ is identified with the space of $G$-fixed linear forms on $V$.

Let $X$ be an $l$-space on which $G$ acts continuously on the left. Let $\lambda$ be the left regular representation of $G$ on $C_{c}^{\infty}(X)$. A $V$-distribution $T$ on $X$ is said to be $\pi$-covariant if

$$
T(f-(\lambda(g) \otimes \pi(g)) f)=0, f \in C_{c}^{\infty}(X) \otimes V .
$$

Lemma 9.1. Let $H$ be a closed subgroup of $G$ and let $(\pi, V)$ be a smooth representation of $G$. Let $T$ be a $\pi$-covariant distribution on $G / H$. Let us denote a left invariant Haar measure on $G$ (resp., $H)$ by $d_{l} g$ (resp., $\left.d_{l} h\right)$. Let us denote the modulus function of $G$ by $\delta_{G}$. It satisfies

$$
\int_{G} f\left(g g_{0}\right) d_{l} g=\delta_{G}\left(g_{0}\right) \int_{G} f(g) d_{l} g, f \in C_{c}(G), g_{0} \in G .
$$

We define a linear map from $C_{c}^{\infty}(G) \otimes V$ to $C_{c}^{\infty}(G / H) \otimes V$ by

$$
M_{H} f(g H):=\int_{H} f(g h) d_{l} h, f \in C_{c}^{\infty}(G) \otimes V .
$$

Then there exists a unique $\eta \in V^{\prime}$ such that

$$
\left\langle T, M_{H} f\right\rangle=\int_{G}\left\langle\pi^{\prime}(g) \eta, f(g)\right\rangle d_{l} g, f \in C_{c}^{\infty}(G) \otimes V .
$$

The linear form $\eta$ will be called the value at 1 of $T$ and denoted $e v_{1} T$ or $T(1)$. Moreover

$$
\pi^{\prime}(h) \eta=\delta_{H}^{-1}(h) \delta_{G}(h) \eta, h \in H .
$$

Notice that $T(1)$ depends on the choice of $d_{l} g, d_{l} h$.

If $G / H$ has a nonzero left $G$-invariant measure, one has

$$
\langle T, f\rangle=\int_{G / H}\left\langle\pi^{\prime}(g) \eta, f(g H)\right\rangle d g H, f \in C_{c}^{\infty}(G / H) \otimes V .
$$

In that case $\eta$ is $H$-invariant and depends only on the choice of the left $G$-invariant measure on $G / H$. 
Proof. Let us assume first that $H=\{1\}$. We remark that $T \in\left(H_{0}\left(G, C_{c}^{\infty}(G) \otimes\right.\right.$ $V))^{\prime}$ where $G$ acts on $C_{c}^{\infty}(G) \otimes V$ by the tensor product $\lambda \otimes \pi$ of the left regular representation with $\pi$. From [3, Prop. 1.13 (iv)], one sees

The map $f \in C_{c}^{\infty}(G) \otimes V \mapsto \int_{G} \pi\left(g^{-1}\right) f(g) d_{l} g$ goes through the quotient to an isomorphism of $H_{0}\left(G, C_{c}^{\infty}(G) \otimes V\right)$ with $V$.

Hence $T$ defines $\eta \in V^{\prime}$ by "transport de structure". One sees that $T$ verifies

$$
\langle T, f\rangle=\int_{G}\left\langle\pi^{\prime}(g) \eta, f(g)\right\rangle d_{l} g, f \in C_{c}^{\infty}(G, V),
$$

which proves our claim when $H=\{1\}$.

In general, we introduce a $V$-distribution $\tilde{T}$ on $G$ by

$$
\langle\tilde{T}, f\rangle=\left\langle T, M_{H} f\right\rangle, f \in C_{c}^{\infty}(G) \otimes V .
$$

Since $T$ is $\pi$-covariant implies that $\tilde{T}$ is $\pi$-covariant, we may apply the first part of the proof to $\tilde{T}$. Now one has

$$
M_{H} R_{h} f=\delta_{H}(h) M_{H} f \in C_{c}^{\infty}(G) \otimes V,
$$

which implies

$$
\left\langle\tilde{T}, R_{h} f\right\rangle=\delta_{H}(h)\langle\tilde{T}, f\rangle, f \in C_{c}^{\infty}(G) \otimes V .
$$

Hence, it follows from (9.2) applied to $\tilde{T}$ that

$$
\left\langle\tilde{T}, R_{h} f\right\rangle=\delta_{H}(h) \int_{G}\left\langle\eta, \pi(g)^{-1} f(g)\right\rangle d_{l} g .
$$

But, again using (9.2), one has

$$
\left\langle\tilde{T}, R_{h} f\right\rangle=\int_{G}\left\langle\pi^{\prime}(g) \eta, f(g h)\right\rangle d_{l} g=\delta_{G}(h) \int_{G}\left\langle\pi^{\prime}(g) \pi^{\prime}\left(h^{-1}\right) \eta, f(g)\right\rangle d_{l} g .
$$

From the preceding equalities one deduces

$$
\delta_{H}(h) \int_{G}\left\langle\eta, \pi(g)^{-1} f(g)\right\rangle d_{l} g=\delta_{G}(h) \int_{G}\left\langle\pi^{\prime}\left(h^{-1}\right) \eta, \pi(g)^{-1} f(g)\right\rangle d_{l} g .
$$

Then (9.1) implies

$$
\pi^{\prime}(h) \eta=\delta_{H}^{-1}(h) \delta_{G}(h) \eta, h \in H
$$

9.2. Bernstein's parameters of finite length smooth modules. The Bernstein's center 9], $Z B(G)$, is identified with an algebra of functions on the set, $\Omega(G)$, of $G$-conjugacy classes of cuspidal pairs, i.e. pairs $(L, \omega)$, where $L$ is a Levisubgroup of $G$ and $\omega$ is a smooth, irreducible cuspidal representation of $L$. Here cuspidal means that the smooth coefficients of the representation are compactly supported modulo the center.

If $(L, \omega)$ is such a pair, we denote its conjugacy class under $G$ by $(L, \omega)_{G}$. If $\Lambda \in \Omega(G)$, we denote the character of $Z B(G)$ given by the evaluation at $\Lambda$ by $\chi_{\Lambda}$ and the kernel of $\chi_{\Lambda}$ by $I_{\Lambda}$. It is a maximal ideal of $Z B(G)$. We say that $\chi_{\Lambda}$ has Bernstein parameter $\Lambda$.

Let $(\pi, V)$ be a smooth $G$-module of finite length. We say that $\left\{\Lambda_{1}, \ldots, \Lambda_{p}\right\} \subset$ $\Omega(G)$ is the set of Bernstein's parameters of $(\pi, V)$, if $V$ splits as a direct sum of 
$G$-modules $V_{1} \oplus \cdots \oplus V_{p}$ such that $V_{i}$ is nonreduced to $\{0\}$ and is annihilated by a power of the ideal $I_{\Lambda_{i}}$ of $Z B(G)$. Then one easily sees

If $\chi$ is an unramified character of $G$ and if the set of Bernstein's parame-

ter of $(\pi, V)$ is equal to $\left.\left\{L_{1}, \omega_{1}\right)_{G}, \ldots,\left(L_{p}, \omega_{p}\right)_{G}\right\}$, the set of Bernstein's parameter of $(\pi \otimes \chi, V)$ is equal to $\left\{\left(L_{1}, \omega_{1} \otimes \chi_{\mid L_{1}}\right)_{G}, \ldots,\left(L_{p}, \omega_{p} \otimes\right.\right.$ $\left.\left.\chi_{\mid L_{p}}\right)_{G}\right\}$.

Let $P$ be a parabolic subgroup of $G$ with Levi subgroup $M$ and let $(\delta, E)$ be a smooth representation of finite length of $M$ with the set of Bernstein's parameters $\left\{\left(L_{1}, \omega_{1}\right)_{M}, \ldots,\left(L_{p}, \omega_{p}\right)_{M}\right\}$, where $\left(L_{i}, \omega_{i}\right)$ is a cuspidal pair for $M$. Then, one has

The set of Bernstein's parameters of $\left(i_{P}^{G} E, i_{P}^{G} \delta\right)$ is $\left\{\left(L_{1}, \omega_{1}\right)_{G}, \ldots,\left(L_{p}, \omega_{p}\right)_{G}\right\}$.

The following is an immediate consequence of the splitting of the category of smooth modules (cf. [2], 25]):

If one has a short exact sequence $0 \rightarrow V_{1} \rightarrow V_{2} \rightarrow V_{3} \rightarrow 0$ of finite length smooth $M^{\prime}$-modules such that $V_{1}$ and $V_{3}$ have disjoint sets of Bernstein's parameters, then $V_{2}$ is isomorphic to the direct sum of $V_{1} \oplus V_{3}$.

9.3. Some results on $\sigma$-parabolic subgroups. In this subsection, we slightly change the notation of the main body of the article.

Lemma 9.2. Let $G$ be a connected algebraic group acting over the nonempty variety $X$.

(i) Let $x \in X$. Then $Y=G x$ is a smooth locally closed subset of $X$.

(ii) There exists at least one closed orbit.

(iii) Let $G_{x}$ be the stabilizer of $x$ in $G$. Then $\operatorname{dim} Y=\operatorname{dim} G-\operatorname{dim} G_{x}$.

Proof. (i) and (ii) follows from [19, Proposition 8.3].

(iii) The morphism $G \rightarrow G . x$ is dominant: this morphism is surjective and $G$ is irreducible, hence $G . x$ is also irreducible and our claim follows from the discussion in the middle of 4, Ch. AG. 8.2]. Then, the assertion on dimensions follows from [4, Ch. AG. 10.1], with $X=G, Y=G . x, W=\{x\}$, and $Z$ the identity component of $G_{x}$.

Lemma 9.3. Let $G$ be a connected algebraic group acting over an irreducible nonsingular variety $X$, with a finite number of orbits. $X$.

If an orbit, $X^{\prime}$, of $G$ in $X$ has the same dimension than $X$, then $X^{\prime}$ is open in

Proof. We use induction on the number of orbits. If this number is 1, our statement is clear. Otherwise, if this number is greater than 1, let $Y$ be a closed orbit in $X$. Then $Y$ is not equal to $X$. As $G$ is connected, $Y$ is irreducible. It follows from [19, Proposition 3.2] that $\operatorname{dim} Y<\operatorname{dim} X$. Then $X \backslash Y$ contains $X^{\prime}$ and is irreducible, as $Y$ is closed and $X$ is irreducible. Moreover the action of $G$ on $X$ induces an action on $X \backslash Y$. One applies the induction hypothesis.

Let $G$ be a connected reductive group defined over a local field $k$ of characteristic different from 2. Let $P, Q$ be two parabolic subgroups of $G$ defined over $k$. Let $G_{k}$ be the set of $k$-points of $G$. We have similar notation for subgroups of $G$. 
Let us show

The $k$-parabolic subgroups $P$ and $Q$ of $G$ are opposed if and only if $P_{k} \cap Q_{k}$ is equal to $M_{k}$ where $M$ is a common Levi to $P$ and $Q$.

If $P$ and $Q$ are opposed, $P \cap Q$ is equal to their common Levi subgroup, $M$, and it is clear that $P_{k} \cap Q_{k}=M_{k}$. Reciprocally if $P_{k} \cap Q_{k}=M_{k}$, then $P \cap Q$ contains the Zariski closure of $M_{k}$ which is equal to $M$ by [4, Corollary 18.3]. By looking to the $k$-parabolic subgroups of $G$ with Levi subgroup $M$, one sees that only the $k$-parabolic subgroup opposed to $P$ satisfies $P_{k} \cap Q_{k}=M_{k}$. This proves our claim.

Hence if $\sigma$ is an involution of $G$ defined over $k$ and $P$ is a parabolic subgroup of $G$ defined over $k, P$ is a $\sigma$-parabolic of $G$ if and only if $P_{k}$ is a $\sigma$-parabolic subgroup of $G_{k}$.

Let us show

If $P$ is a $\sigma$-parabolic subgroup of $G, \underline{p}+\underline{h}=\underline{g}$, where $\underline{g}$ (resp., $\underline{p}$ ) is the

Lie algebra of $G$ (resp., $P$ ) and $\underline{h}$ is the Lie algebra of the fixed point group of $\sigma$.

As $P$ is a $\sigma$-parabolic subgroup of $G, \underline{p}+\sigma(\underline{p})=\underline{g}$. Hence any $X \in \underline{g}$ is of the form $Y+\sigma(Z)$ with $Y, Z \in \underline{p}$. Hence, one has

$$
X=Y-Z+(Z+\sigma(Z)) .
$$

The result follows from the fact that $\underline{h}$ is equal to the fixed point set of $\sigma$ in $\underline{g}$ (cf. [26, proof of Lemma 2.4]). Let $H$ be an open subgroup, defined over $k$, of the fixed point group of $\sigma$. We will show

Let $P$ be a $\sigma$-parabolic subgroup of $G$ defined over $k$. Then: a) $H P$ is open in $G$, b) $H_{k} P_{k}$ is open in $G_{k}$.

Assertion a) follows from [18, Lemma 4.8] and assertion b) reduces to the case where $P$ is a minimal $\sigma$-parabolic subgroup of $G$ defined over $k$. In that case it follows from [18, Definition 13.1 and Proposition 13.4].

Lemma 9.4. Let $P$ be a $\sigma$-parabolic subgroup of $G$ defined over $k$. Let $x \in G_{k}$.

The following conditions are equivalent:

(i) $H_{k} x P_{k}$ is open in $G_{k}$.

(ii) $H x P$ is open in $G$.

(iii) $x \in H P$.

(iv) $x P x^{-1}$ is a $\sigma$-parabolic subgroup of $G$ defined over $k$.

Proof. (i) implies (ii): Let $P^{\prime}$ be a minimal $k$-parabolic subgroup of $G$ contained in $P$. There are finitely many $\left(H_{k}, P_{k}^{\prime}\right)$-double cosets in $G_{k}$ (cf. [18, Corollary 6.16]). Hence $H_{k} x P_{k}$ contains an open $\left(H_{k}, P_{k}^{\prime}\right)$-double coset, $H_{k} x^{\prime} P_{k}$, with $x^{\prime}=x p$ and $p \in P_{k}$. From [17, Proposition 3.5], one sees that $x^{\prime} . P^{\prime}$ is contained in a minimal $\sigma$-parabolic subgroup of $G$, hence $H x^{\prime} P^{\prime}$ is open in $G$ by [18, Lemma 4.8]. Hence $H x^{\prime} P=H x P$ is open in $G$ and hence (i) implies (ii).

(ii) implies (iii): As the union of the closures of the open $(H, P)$-double coset in $G$ is equal to $G$ and as $G$ is connected, hence irreducible, there is only one open $(H, P)$-double coset in $G$. From (9.8) a), one knows that $H P$ is open. Hence (ii) implies (iii).

(iii) implies (iv) because the conjugation by an element of $H$ preserves the set of $\sigma$-parabolic subgroups of $G$.

(iv) implies (i) follows from (9.8) b). 


\section{Compact open subroups of Lie groups over $\boldsymbol{F}$}

Let $G$ be a Lie group over $\mathbf{F}$ in the sense of [6, Ch. III.1, Definition 1] and let $\underline{g}$ be its Lie algebra.

We will use an idea given by Deligne in [9, top of p. 16]. We fix an analytic bijective map $\psi: V \rightarrow W$ between an open neighborhood $V$ of 0 in $g$ to an open neighborhood $W$ of 1 in $G$. We assume that its differential at 0 is the identity. Such a map will be called a good chart at 1 for $G$.

Lemma 10.1. Let $\mathfrak{P}$ be the the maximal ideal of the ring of integers, $\mathfrak{O}$ of $\mathbf{F} . W e$ fix a basis $\left(X_{j}\right)$ of $g$.

(i) Let $\Lambda_{n} g$ (resp. $\Lambda g$ ) be the $\mathfrak{P}^{n}$-module (resp. $\mathfrak{O}$-module) generated by $\left(X_{j}\right)$. It is a basis of neighborhoods of 0 in $\mathfrak{g}$. Let $J_{n}$ be the image of $\Lambda_{n} \underline{g}$ under $\psi$, which is defined for $n$ large enough.

Then for $n$ large enough, $J_{n}$ is a compact open subgroup of $G$.

(ii) If $J_{n}^{\prime}$ is defined with another good chart $\psi^{\prime}$, one has $J_{n}=J_{n}^{\prime}$ for $n$ large enough.

(iii) Let $\Theta$ be a family of automorphisms of the Lie group $G$ whose differential preserves $\Lambda$. Then it preserves $\Lambda_{n} \underline{g}$ for all $n \in \mathbb{N}^{*}$. We assume moreover that $\Theta$ has the structure of a compact analytic manifold over $\mathbf{F}$ and that the map $\Theta \times G \rightarrow$ $G,(\theta, g) \mapsto \theta(g)$ is analytic. Then for $n$ large enough, $J_{n}$ is invariant under every $\theta \in \Theta$.

(iv) Let us assume that we are given three closed Lie subgroups $G_{1}, G_{2}, G_{3}$ of $G$ and vector subspaces $\underline{g}_{i}^{\prime} \subset \underline{g}_{i}, i=1,2,3$, of $\underline{g}$ such that $\Lambda \underline{g}=\left(\Lambda \underline{g} \cap \underline{g}_{1}^{\prime}\right) \oplus(\Lambda \underline{g} \cap$ $\left.\underline{g}_{2}^{\prime}\right) \oplus\left(\Lambda \underline{g} \cap \underline{g}_{3}^{\prime}\right)$. Then for $n$ large enough one has

$$
J_{n}=\left(J_{n} \cap G_{1}\right)\left(J_{n} \cap G_{2}\right)\left(J_{n} \cap G_{3}\right) .
$$

Proof. (i) The fact that $J_{n}$ is compact and open follows from the fact that $\Lambda_{n} \underline{g}$ is compact and open. We choose $n$ large enough so that $J_{n} J_{n} \subset W$.

Let us denote by $x_{j}$ the $j$-th coordinate map on $\underline{g}$. We define

$$
|X|=\operatorname{Sup}_{j}\left|x_{j}(X)\right|_{\mathbf{F}}
$$

Let $X, X^{\prime} \in \Lambda_{n} \underline{g}$, and let us study $x_{j}\left(\psi^{-1}\left(\psi(X) \psi\left(X^{\prime}\right)\right)\right)$. By our hypothesis on $\psi$ and from the formula of the differential of the product in $G$, one sees that the differential of this map of $\left(X, X^{\prime}\right)$ is simply $\left(X, X^{\prime}\right) \mapsto x_{j}(X)+x_{j}\left(X^{\prime}\right)$. The definition of the differential shows that

$$
x_{j}\left(\psi^{-1}\left(\psi(X) \psi\left(X^{\prime}\right)\right)\right)=x_{j}(X)+x_{j}\left(X^{\prime}\right)+\operatorname{Sup}\left(|X|,\left|X^{\prime}\right|\right) \varepsilon\left(X, X^{\prime}\right),
$$

where $\varepsilon\left(X, X^{\prime}\right)$ tends to zero if $\left(X, X^{\prime}\right)$ tends to $(0,0)$. Let $n_{0}$ be large enough such that $\left|\varepsilon\left(X, X^{\prime}\right)\right|<1$ for $X, X^{\prime}$ in $\Lambda_{n_{0}} g$. One deduces from the above equality that for $n \in \mathbb{N}$ larger than $n_{0}, j j^{\prime} \in J_{n}$ for all $j, j^{\prime} \in J_{n}$. Similarly one sees that $j^{-1} \in J_{n}$ if $j \in J_{n}$ and $n$ is larger than some $n_{0}^{\prime}$. Then (i) follows.

(ii) One proceeds as in (i), by considering the map $X \mapsto x_{j}\left(\psi^{-1}\left(\psi(X)\left(\psi^{\prime}(X)\right)^{-1}\right)\right)$, whose differential at 0 is equal to zero. Arguing as in (i), for $n$ large one sees that $\psi(X)\left(\psi^{\prime}(X)\right)^{-1} \in J_{n+1}$ if $X \in \Lambda_{n} \underline{g}$. Hence if $j \in J_{n}$, we have found $j^{\prime} \in J_{n}^{\prime}$ such that $j\left(j^{\prime}\right)^{-1} \in J_{n+1}$. Using the fact that $J_{n}^{\prime}$ is a group, and proceeding inductively, we find a sequence $\left(j_{p}^{\prime}\right)$ in $J_{n}^{\prime}$ such that $j\left(j_{p}^{\prime}\right)^{-1} \in J_{n+p}^{\prime}$. Hence $j_{p}^{\prime}$ converges to $j$. But $J_{n}^{\prime}$ is compact, hence $j \in J_{n}^{\prime}$ and $J_{n} \subset J_{n}^{\prime}$. The reverse inclusion is proved similarly. This proves (ii). 
(iii) By compactness, one is reduced to prove that for every $\theta_{0} \in \Theta$, there exist a neighborhood $V\left(\theta_{0}\right)$ of $\theta_{0}$ in $\Theta$ and $n_{0} \in \mathbb{N}$ such that for all $n \in \mathbb{N}$ greater than $n_{0}$ and for all $\theta \in V\left(\theta_{0}\right)$, one has $\theta\left(J_{n}\right) \subset J_{n}$. We fix $\theta_{0}$. We again denote the differential at 1 of $\theta \in \Theta$ by $\theta$. Shrinking $V$ and $\Theta$ if necessary, let us consider the analytic map from $(\Theta, V)$ to $g,(\theta, X) \mapsto \psi^{-1}\left[\theta(\psi(X))(\psi(\theta(X)))^{-1}\right]$. By looking to partial derivatives, one sees that its differential at $\left(\theta_{0}, 0\right)$ is equal to zero. One sees as above that it implies that for $\theta$ in a small neighborhood $V\left(\theta_{0}\right)$ of $\theta_{0}$ and for $n$ large enough one has

$$
\theta(\psi(X))(\psi(\theta(X)))^{-1} \in J_{n}, X \in \Lambda_{n} \underline{g} .
$$

As $\theta(X) \in \Lambda_{n} \underline{g}$, this implies $\theta(\psi(X)) \in J_{n}$. This proves (iii).

(iv) Let $\psi_{i}$ be a good chart at zero of $G_{i}, i=1,2,3$. From the first part of the lemma, one can use the following map $\psi$ to study $J_{n}$ :

$$
\psi\left(Y_{1}+Y_{2}+Y_{3}\right)=\psi_{1}\left(Y_{1}\right) \psi_{2}\left(Y_{2}\right) \psi_{3}\left(Y_{3}\right), Y_{i} \in \underline{g}_{i}^{\prime}, i=1,2,3 .
$$

One easily sees that $\Lambda_{n} \underline{g}=\left(\Lambda_{n} \underline{g} \cap \underline{g}_{1}^{\prime}\right) \oplus\left(\Lambda_{n} \underline{g} \cap \underline{g}_{2}^{\prime}\right) \oplus\left(\Lambda_{n} \underline{g} \cap \underline{g}_{3}^{\prime}\right)$. From the definition of $J_{n}$ it is clear that

$$
J_{n} \subset\left(J_{n} \cap G_{1}\right)\left(J_{n} \cap G_{2}\right)\left(J_{n} \cap G_{3}\right) .
$$

The reverse inclusion being clear, this proves the lemma.

Lemma 10.2. If $\underline{G} \subset G L(n)$ is a linear algebraic group defined over $\mathbf{F}$, the group $G=\underline{G}(\mathbf{F})$ has a structure of analytic Lie group, whose Lie algebra $g$ is the Lie algebra, $g(\mathbf{F})$, of $\mathbf{F}$-points of the Lie algebra of $\underline{G}$.

Proof. The following fact seems to be well known but by lack of reference, we give a proof. We thank Joseph Bernstein, Bertrand Lemaire and Jean-Pierre Labesse for discussions on this fact.

Let $X$ be an affine irreducible variety. Let us assume that $X$ is nonsingular and defined over $\mathbf{F}$ and let $\Omega$ be the algebraic closure of $\mathbf{F}$. Let us assume that $X$ is a Zariski closed set in $\Omega^{n}$. Let $x \in X(\mathbf{F})$.

(10.1) coefficients in $\mathbf{F}$ such that the rank of their differentials at $x$ is equal to the codimension of $X$ in $\Omega^{n}$ and to $p$.

(b) If $f_{1}, \ldots, f_{p}$ are as above, let $V\left(f_{1}, \ldots, f_{p}\right)$ be the set of their common zeroes. There exists an open subset $O$ in $\Omega^{n}$ such that $x \in O$ and such that $X \cap O$ is is equal to $V\left(f_{1}, \ldots, f_{p}\right) \cap O$.

(a) follows from the definition of nonsingular points in [15, I.5] and from the fact that the ideal of functions vanishing on $X$ is generated by polynomials with coefficients in $\mathbf{F}$.

(b) Let $Y=V\left(f_{1}, \ldots, f_{p}\right)$ and let $O^{\prime}$ be the open set of elements of $\Omega^{n}$ such that the rank of the differential of $f_{1}, \ldots, f_{p}$ is of rank $p$. It is open in $\Omega^{n}$ and it contains $x$. By [15, Theorem I.5.3], one knows that

The rank $r$ of the Jacobian matrix, at a point of $y \in Y$, associated to polynomial functions vanishing on $Y$, is at most $n-\operatorname{dim}(Y)$, i.e. $\operatorname{codim}(Y) \geq r$.

Applying this to $f_{1}, \ldots, f_{p}$ one has $\operatorname{codim}(Y) \geq p$, hence the codimension of any irreducible component of $Y$ is of codimension greater than or equal to $p$. On the other hand, from the definition of $Y$ by $p$ equations and [19, 3.4 Corollary B], every 
irreducible component of $Y$ has codimension at most $p$. Hence every irreducible component of $Y$ is of dimension $n-p$. Then from the definition of a nonsingular point in [15, I.5] and (10.2), one sees that $x$ is nonsingular in $Y$.

A simple point in the sense of [4, AG.17.1] is exactly a nonsingular point in the sense of [15], due to [15, Theorem I.5.1]. Hence, by [4, Theorem 17.1], one sees that $x$ belongs to only one irreducible component of $Y$. Let $Z$ be the irreducible component of $Y$ which contains $x$ and let $O^{\prime \prime}$ be the complement in $\Omega^{n}$ of the (finite) union of the other irreducible components of $Y$. It is open in $\Omega^{n}$ and contains $x$.

Let us show that $O:=O^{\prime} \cap O^{\prime \prime}$ satisfies (b). In fact $X^{\prime}=X \cap O$ is contained in $Z^{\prime}=Z \cap O$ and has the same dimension. It contains $x$. Moreover as $X$ (resp. $Z$ ) is irreducible, $X^{\prime}$ and $Z^{\prime}$ are irreducible [4, AG.1.1]. Hence from [19, Proposition 3.2], one has $X^{\prime}=Z^{\prime}$. This proves (10.1).

Let $f_{1}, \ldots, f_{p}$ be a set of polynomials, with coefficients in $\mathbf{F}$, as in (10.1) for $X=\underline{G}$. Then we consider the map $h: \mathbf{F}^{n} \rightarrow \mathbf{F}^{n}, x \mapsto\left(f_{1}(x), \ldots, f_{p}(x), x_{1}(x), \ldots x_{n-p}(x)\right)$, where the affine coordinates $\left(x_{1}, \ldots, x_{n}\right)$ in $\mathbf{F}^{n}$ are equal to zero at the neutral element 1 of $G$ and are chosen such that the differential of $h$ at 1 is bijective. The Inverse Function Theorem (cf. [28, LG.2.10]) gives a local analytic inverse to this map, $\phi$.

Let $\psi$ be the map defined on an open neighborhood $V$ of $(0, \ldots, 0)$ in $\mathbf{F}^{n-p}$ by $\psi(y)=\phi((0, \ldots, 0), y)$. From (10.1) and from the fact that $\phi$ is a homeomorphism, one sees that if $V$ is small enough, $\psi$ is a homeomorphism from $V$ onto an open neighborhood of 1 in $G$. The differential at 0 of $\psi$ is injective and its image is a subspace of the space of common zeroes in $\mathbf{F}^{n}$ of the differential at 1 of $f_{1}, \ldots, f_{p}$. The space of common zeroes in $\Omega^{n}$ of the differential at 1 of $f_{1}, \ldots, f_{p}$ is the tangent space at 1 of $\underline{G}$, hence is equal to $\underline{g}$. For reasons of dimension this implies that the image of the differential at 0 of $\psi$ is equal to $g(\mathbf{F})$.

The map $\psi$ gives one chart of $G$ at 1 . Then by translation this gives a chart at every point of $G$. The analyticity of the change of charts follows from the analyticity of $\phi$ and its inverse. Similarly one proves that $G$ has a structure of Lie groups. It is even an analytic Lie subgroup of $G L(n, \mathbf{F})$. The Lie algebra of $G$ is the image of the differential at 0 of $\psi$. Hence the Lie algebra of $G$ is $\underline{g}(\mathbf{F})$.

\section{ACKNOWLEDGEMENTS}

The authors warmly thank the referees for numerous helpful remarks and comments. The second author thanks Joseph Bernstein for enlighting conversations during the elaboration of this work. The authors also thank Jean-Pierre Labesse and Bertrand Lemaire for their decisive help on algebraic groups.

\section{REFERENCES}

[1] E. P. van den Ban, The principal series for a reductive symmetric space. I. H-fixed distribution vectors, Ann. Sci. École Norm. Sup. (4) 21 (1988), no. 3, 359-412. MR974410 (90a:22016)

[2] Bernstein J., Second Adjointness Theorem for representations of reductive p-adic groups, unpublished manuscript, web page of Joseph Bernstein.

[3] Philippe Blanc and Patrick Delorme, Vecteurs distributions H-invariants de représentations induites, pour un espace symétrique réductif p-adique $G / H$ (French, with English and French summaries), Ann. Inst. Fourier (Grenoble) 58 (2008), no. 1, 213-261. MR2401221 (2009e:22015)

[4] Armand Borel, Linear algebraic groups, 2nd ed., Graduate Texts in Mathematics, vol. 126, Springer-Verlag, New York, 1991. MR1102012 (92d:20001) 
[5] Armand Borel and Jacques Tits, Groupes réductifs (French), Inst. Hautes Études Sci. Publ. Math. 27 (1965), 55-150. MR0207712 (34 \#7527)

[6] Bourbaki, Groupes et algébres de Lie. Chapitres 2 et 3, Eléments de Mathématique XXXVII, Herman, Paris 1972.

[7] Colin J. Bushnell, Representations of reductive p-adic groups: localization of Hecke algebras and applications, J. London Math. Soc. (2) 63 (2001), no. 2, 364-386, DOI 10.1017/S0024610700001885. MR1810135 (2001m:22034)

[8] W. Casselman, Introduction to the theory of admissible representations of $p$-adic reductive groups, http://www.math.ubc.ca/ cass/research.html.

[9] J. N. Bernstein, Le "centre" de Bernstein (French), Representations of reductive groups over a local field, Travaux en Cours, Hermann, Paris, 1984, pp. 1-32. Edited by P. Deligne. MR771671 (86e:22028)

[10] Patrick Delorme, Intégrales d'Eisenstein pour les espaces symétriques réductifs: tempérance, majorations. Petite matrice B (French, with English summary), J. Funct. Anal. 136 (1996), no. 2, 422-509, DOI 10.1006/jfan.1996.0035. MR.1380660 (96m:22027)

[11] Patrick Delorme, The Plancherel formula on reductive symmetric spaces from the point of view of the Schwartz space, Lie theory, Progr. Math., vol. 230, Birkhäuser Boston, Boston, MA, 2005, pp. 135-175, DOI 10.1007/0-8176-4426-1_3. MR2099395

[12] Patrick Delorme, Constant term of smooth $H_{\psi}$-spherical functions on a reductive p-adic group, Trans. Amer. Math. Soc. 362 (2010), no. 2, 933-955, DOI 10.1090/S0002-9947-0904925-3. MR2551511(2011d:22016)

[13] Patrick Delorme, Théorème de Paley-Wiener pour les fonctions de Whittaker sur un groupe réductif p-adique (French, with English and French summaries), J. Inst. Math. Jussieu 11 (2012), no. 3, 501-568, DOI 10.1017/S1474748011000193. MR2931317

[14] P. Delorme, Formule de Plancherel pour les fonctions de Whittaker sur un groupe réductif $p$-adique, to appear in Ann. Inst. Fourier.

[15] Robin Hartshorne, Algebraic geometry, Springer-Verlag, New York, 1977. Graduate Texts in Mathematics, No. 52. MR0463157 (57 \#3116)

[16] Volker Heiermann, Une formule de Plancherel pour l'algèbre de Hecke d'un groupe réductif p-adique (French, with French summary), Comment. Math. Helv. 76 (2001), no. 3, 388-415, DOI 10.1007/PL00013213. MR1854691 (2002g:22023)

[17] A. G. Helminck and G. F. Helminck, A class of parabolic k-subgroups associated with symmetric k-varieties, Trans. Amer. Math. Soc. 350 (1998), no. 11, 4669-4691, DOI 10.1090/S00029947-98-02029-7. MR1443876 (99g:20082)

[18] A. G. Helminck and S. P. Wang, On rationality properties of involutions of reductive groups, Adv. Math. 99 (1993), no. 1, 26-96, DOI 10.1006/aima.1993.1019. MR1215304(94d:20051)

[19] James E. Humphreys, Linear algebraic groups, Springer-Verlag, New York, 1975. Graduate Texts in Mathematics, No. 21. MR0396773 (53 \#633)

[20] Shin-ichi Kato and Keiji Takano, Subrepresentation theorem for p-adic symmetric spaces, Int. Math. Res. Not. IMRN 11 (2008), Art. ID rnn028, 40, DOI 10.1093/imrn/rnn028. MR2428854 (2009i:22021)

[21] Shin-ichi Kato and Keiji Takano, Square integrability of representations on p-adic symmetric spaces, J. Funct. Anal. 258 (2010), no. 5, 1427-1451, DOI 10.1016/j.jfa.2009.10.026. MR2566307 (2011b:22028)

[22] A. W. Knapp and E. M. Stein, Intertwining operators for semisimple groups. II, Invent. Math. 60 (1980), no. 1, 9-84, DOI 10.1007/BF01389898. MR582703(82a:22018)

[23] Nathalie Lagier, Terme constant de fonctions sur un espace symétrique réductif p-adique (French, with English and French summaries), J. Funct. Anal. 254 (2008), no. 4, 1088-1145, DOI 10.1016/j.jfa.2007.07.012. MR2381204(2009d:22013)

[24] Toshihiko Matsuki, Closure relations for orbits on affine symmetric spaces under the action of minimal parabolic subgroups, Representations of Lie groups, Kyoto, Hiroshima, 1986, Adv. Stud. Pure Math., vol. 14, Academic Press, Boston, MA, 1988, pp. 541-559. MR1039852 (91c:22014)

[25] David Renard, Représentations des groupes réductifs p-adiques (French), Cours Spécialisés [Specialized Courses], vol. 17, Société Mathématique de France, Paris, 2010. MR2567785 (2011d:22019) 
[26] R. W. Richardson, Orbits, invariants, and representations associated to involutions of reductive groups, Invent. Math. 66 (1982), no. 2, 287-312, DOI 10.1007/BF01389396. MR656625 (83i:14042)

[27] François Sauvageot, Principe de densité pour les groupes réductifs (French, with English and French summaries), Compositio Math. 108 (1997), no. 2, 151-184, DOI 10.1023/A:1000216412619. MR.1468833 (98i:22025)

[28] Jean-Pierre Serre, Lie algebras and Lie groups, Lectures given at Harvard University, vol. 1964, W. A. Benjamin, Inc., New York-Amsterdam, 1965. MR0218496 (36 \#1582)

[29] J.-L. Waldspurger, La formule de Plancherel pour les groupes p-adiques (d'après HarishChandra) (French, with French summary), J. Inst. Math. Jussieu 2 (2003), no. 2, 235-333, DOI 10.1017/S1474748003000082. MR.1989693 (2004d:22009)

Aix Marseille Université, CNRS, Centrale Marseille, I2M, UMR7373, 163 Avenue de Luminy, Marseille Cedex 09, France

E-mail address: carmona@iml.univ-mrs.fr

Aix Marseille Université, CNRS, Centrale Marseille, I2M, UMR7373, 163 Avenue de Luminy, Marseille Cedex 09, France

E-mail address: delorme@iml.univ-mrs.fr 